\title{
MODULI SPACES AND FREDHOLM THEORY FOR PSEUDOHOLOMORPHIC SUBVARIETIES ASSOCIATED TO SELF-DUAL, HARMONIC 2-FORMS*
}

\author{
C. H. TAUBES ${ }^{\dagger}$
}

A compact, oriented Riemannian 4-manifold whose intersection form is not negative definite has a non-trivial closed, self-dual 2 -form. Such a form defines a symplectic structure away from its zero set, and the metric can be used to defines a compatible almost complex structure. Although this almost complex structure is singular across the zero set of the given 2 -form, one can none-the-less study the associated pseudoholomorphic subvarieties in the compliment of the zero set. In this regard, it is natural to restrict attention to those which have the following three properties: First, they are closed subsets of the compliment of the zero set of the given self-dual 2-form. Second, they are submanifolds except at a set which can be empty, but is at worst countable and non-accumulating. Third, the given self-dual 2-form has finite integral over the subvariety. With regard to the second point, remark that the condition of pseudoholomorphicity is no more nor less than the requirement that the almost complex structure preserve the tangent space at all manifold points. With regard to the third point, remember that a pseudoholomorphic variety is naturally oriented at its manifold points by the restriction of the symplectic form.

The main theorem in [T1] asserts that such pseudoholomorphic subvarieties exist when the given 4-manifold has a non-zero Seiberg-Witten invariant. Meanwhile, [T2] discusses aspects of the regularity theory of pseudoholomorphic subvarieties when the metric and self-dual 2-form have a certain prescribed structure near the zero set of the 2-form. This structure is also described in (1.1) below as it is assumed here as well. In the regard, note that there are no obstructions to finding metrics and forms which obey the constraints from [T2].

This article serves as a sequel of sorts to [T1] and [T2] as it constructs a moduli space for a certain subset of pseudoholomorphic subvarieties. To describe this subset, digress momentarily to note that the aforementioned, prescribed structure for the metric and 2-form near the latter's zero set guarantees that this zero set is a disjoint union of embedded circles with a distinguished 2-plane subbundle in its normal bundle. With the digression now ended, the subset of pseudoholomorphic subvarieties in question consists of those which approach the zero set of the self-dual form with one tangent asymptotic to the distinguished 2-plane subbundle. The following drawing illustrates a one sheeted example:

*Received March 1, 1999; accepted for publication March 20, 1999.

†Department of Mathematics, Harvard University, Cambridge, MA 02138, USA (chtaubes@ math.harvard.edu). The research is supported in part by the National Science Foundation. 


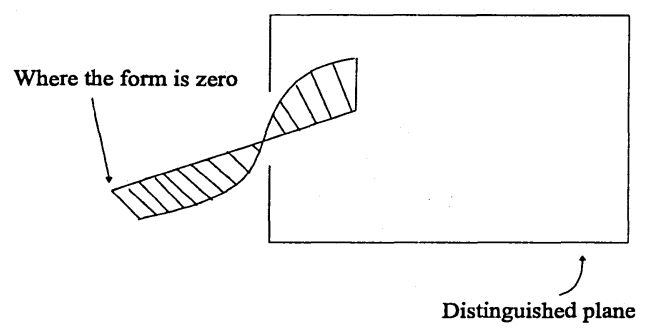

3-d slice along the form's zero set

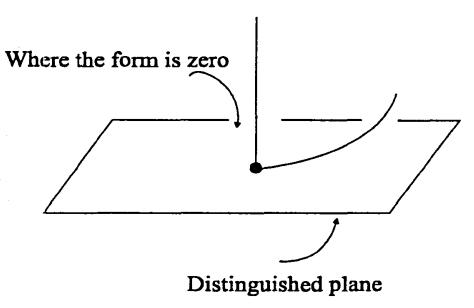

3-d slice across the form's zero set

A subvariety with one tangent asymptotic to the distinguished 2-plane bundle

The subvarieties whose sheets all conform to the preceding illustration are called boundary regular here and are described in more detail by Definition 1.2. There are examples of boundary regular pseudoholomphic subvarieties; and it is most probably the case, though not yet proved, that such varieties (including those from [T1]) are generic in a suitable sense.

In any event, this article constructs stratified moduli spaces for the boundary regular pseudoholomphic subvarieties. The following theorem summaries the basic picture:

Theorem. The set of pseudoholomphic subvarieties has a natural topology for which the subset, $\mathcal{M}_{b}$, of boundary regular subvarieties is open. Moreover, the latter set is a countable, nested union $\mathcal{M}_{b}=\mathcal{M}_{b, 0} \supset \mathcal{M}_{b, 1} \supset \cdots \supset \mathcal{M}_{b, k} \subset \cdots$, where, for each $k \geq 0, \mathcal{M}_{b, k+1} \subset \mathcal{M}_{b, k}$ is closed and each $C \in \mathcal{M}_{b, k}-\mathcal{M}_{b, k+1}$ has an open neighborhood in $\mathcal{M}_{b, k}$ which is homeomorphic to the zero set of a smooth map between balls about the origin in the kernel and cokernel of a Fredholm operator.

(Definition 2.3, below, gives a complete definition of $\left\{\mathcal{M}_{b, k}\right\}_{k \geq 0}$ )

In the case where the closed, self dual form is nowhere zero, the preceding conclusion forms a key part of the foundation of the theory of pseudoholomorphic curves on symplectic manifolds. (See, e.g., $[\mathrm{Gr}],[\mathrm{MS}]$.) Thus, this article serves to extend a part of the foundational structure to the context of pseudoholomorhpic curves for self dual forms.

As an aside, note that there is reasonable evidence that the compliment of the set of boundary regular subvarieties has, in some generic sense, codimension at least 1 relative to nearby boundary regular subvarieties. In any event, this compliment will not be discussed here, but in a planned sequel.

At the time of this writing, the author knows of three 4-manifold applications of this self-dual, pseudoholomorphic geometry. The first is the classical case where the self-dual 2-form has no zero set and thus makes the manifold symplectic. See [T3], [T4] for a summary of applications in the symplectic case. The second case assumes that the four-manifold has a non-trivial circle action, in which case one can study an $S^{1}$ invariant version of the story. This turns out to be equivalent to studying a certain form of circle valued Morse theory on a three manifold. The reader is referred to [HL1], [HL2] and [Tu]. The third is provided by the main theorem in [T1] which asserts the following: If $b^{2+}(X) \geq 1$ and $X$ has a non-zero Seiberg-Witten invariant, then there are pseudoholomorphic subvarieties in the compliment of a closed, self-dual form's zero set which have intersection number 1 with every 2 -sphere that links said zero set.

In any event, [T4] also contains some rash speculations by the author about possible directions for the general 4-manifold story. In this vein, note that the fact 
that the boundary regular subvarieties form a moduli space with a model given by Fredholm operators backs the suggestion from [T1] and [T4] that the Seiberg-Witten invariants of the four manifold can be obtained by counting the pseudoholomorphic subvarieties which form the homological boundary of the closed form's zero set.

The reader is also encouraged to study the well developed approach of Hofer, Wysocki and Zehnder to pseudoholomorphic subvarieties for singular forms. Their work is described in a series of articles which begin with [HWZ] and are summarized and referenced in Hofer's 1998 ICM plenary lecture $[\mathrm{H}]$.

1. The background. With the preceding preamble complete, it is time to describe in more detail the background and setting for the Theorem in the Introduction. In particular, the description of the setting starts with a formal restatement of the definition of a pseudoholomorphic subvariety:

DEFINITION 1.1. Let $\omega$ and $J$ be a pair consisting of a symplectic form and a compatible almost complex structure on a smooth, oriented (possibly non-compact) 4manifold. A subset $C$ in the four manifold is called a pseudoholomorphic subvariety when the following conditions are met:

- $C$ is closed.

- There is a countable set $\Lambda \subset C$ without accumulation points and with the property that $C-\Lambda$ is a smooth, dimension 2 submanifold whose tangent space is mapped by $J$ to itself.

- $\int_{C} \omega<\infty$.

Equivalently, $C$ is a pseudoholomorphic subvariety when there exists a complex curve $C_{0}$ with a proper, pseudoholomorphic map into the 4-manifold which is 1-1 except on a countable, nonaccumulating set, and which pulls back the symplectic form $\omega$ to a finite volume 2-form.

With the notion of a pseudoholomorphic subvariety understood, return now to the case where the 4-manifold in question is the compliment of the zero set of a closed, self-dual 2-form on a compact Riemannian 4-manifold. (Below, the 4-manifold is denoted by $X$ ). A digression follows now to explain certain geometric constraints which will be assumed in this paper.

To start the digression, remark, as above, that the discussion here follows that in [T2] by assuming certain constraints on the self-dual 2-form and the metric near the zero set of the former. In particular, assume that the form, $\omega$, vanishes transversely as a section of the metric's bundle of self-dual 2-forms. It then follows (see, e.g., [T2]) that the normal bundle to $Z$ in $X$ splits naturally as the direct sum of a real line bundle and a real 2-plane bundle. The former can either be orientable or not along each component of $Z$. This line bundle will be called the ' $z$-axis bundle' for reasons that should be evident below. (Note that Gompf shows that the mod (2) parity of the number of components of $Z$ which have orientable $z$-axis bundle is opposite the mod (2) parity of the sum of the first Betti number of $X$ with the self-dual part of the second Betti number of $X$.)

With respect to the behavior of the metric and form $\omega$ near $Z$, assume in addition that each component of the zero set, $Z$, of $\omega$ has a tubular neighborhood with the property that either it or its double cover has coordinates $(t, x, y, z)$ where $t \in S^{1}=$ $\mathbb{R} /(2 \pi \mathbb{Z})$ and where $(x, y, z)$ takes values in some positive radius ball about the origin in $\mathbb{R}^{3}$. Furthermore, assume that in these coordinates,

- The set where $Z=\omega^{-1}(0)$ corresponds to the $t$-axis.

- The given metric is Euclidean, $d t^{2}+d x^{2}+d y^{2}+d z^{2}$. 
- $\omega=x d t \wedge d x+y d t \wedge d y-2 z d t \wedge d z+x d y \wedge d z-y d x \wedge d z-2 z d x \wedge d y$.

- The coordinates $(t, z, x, y)$ are defined on $Z$ 's tubular neighborhood as opposed to a double cover precisely when the $z$-axis line bundle is orientable.

(Note that in the coordinates of (1.1), the $z$-axis line bundle coincides with the $z$-axis.)

In (1.1) and below, neither the form $\omega$ nor the metric are distinguished notationally from their pull-backs to the double cover of the given neighborhood of $Z$. As noted in [T2], the constraints in (1.1) are not hard to satisfy. To simplify the notation in the subsequent discussions, the following convention will be employed: Unless stated to the contrary, the radius out from $Z$ on which (1.1) holds will be taken to be 1. Also, the length of each component of $Z$ will be assumed to equal $2 \pi$. Except for the notational simplifications, neither of these two assumptions has any substantive affect on the subsequent discussion.

As in [T2], it proves useful to introduce the functions.

- $f=2^{-1}\left(x^{2}+y^{2}-2 z^{2}\right)$.

- $h \equiv\left(x^{2}+y^{2}\right) z$.

- $g \equiv\left(x^{2}+y^{2}+4 z^{2}\right)^{1 / 2}$

as well as the standard radial coordinates $\rho \equiv\left(x^{2}+y^{2}\right)^{1 / 2}$ and $\varphi=\arctan (y / x)$ for the $x$ - $y$ plane. Also introduce the function $r=\left(t^{2}+x^{2}+y^{2}+z^{2}\right)^{1 / 2}$ to measure distance to the origin. With regard to the introduction of the functions in (1.2), the point is that the metric line element and the form $\omega$ have the following nice form in the coordinates $(t, f, h, \varphi)$ :

- $d s^{2}=d t^{2}+g^{-2}\left(d t^{2}+\rho^{-2} d h^{2}\right)+\rho^{2} d \varphi^{2}$

- $\omega=d t \wedge d f+d \varphi \wedge d h$

With the digression now at an end, here is a summary of some of [T2]'s result about pseudoholomorphic subvarieties in $X-C$ : If the metric and closed-self dual form obey the constraints in (1.1), then a pseudoholomorphic subvariety $C$ enjoys the following regularity properties:

- The subvariety has finite area.

- There is a finite and possibly empty set $Z_{S} \subset Z$ which is characterized by the property that each point in $Z-Z_{S}$ has a ball neighborhood in $X$ which intersects $C$ as a finite set of components where the closure of each is a real analytically embedded half disk whose straight edge coincides with $Z$.

- If $\tau \in Z-Z_{S}$, then each component of the intersection of $C$ with a small radius ball centered at $\tau$ is described by one of the following two statements:

(a) The component is a half-disk in the $t$ - $z$ plane.

(b) The component is a graph over the $(t, \rho)$ plane, parameterized as $(t, \rho, z(t, \rho), \varphi(t, \rho))$ with

$$
\begin{aligned}
& \varphi(t, \rho)=\varphi_{0}(t)+\mathcal{O}\left(\rho^{2}\right) \\
& z(t, \rho)=\frac{1}{4} \frac{d \varphi_{0}}{d t}(t) \rho^{2}+\mathcal{O}\left(\rho^{4}\right)
\end{aligned}
$$

where $\varphi_{0}(\cdot)$ is an analytic function of $t$ which is defined near $t=\tau$. Moreover, the higher order terms in the preceding expansions are also determined by the function $\varphi_{0}$.

- Let $q_{+}$and $q_{-}$denote the respective number of components of the intersection of $C$ with a sufficiently small radius ball about a point in $Z-Z_{S}$ which lie, respectively, in the $\pm z>0$ portion of the $t$ - $z$ plane. Let $p$ denote the 
corresponding number of components which are described by Statement b, above. Then $q_{ \pm}$and $p$ are constant on each component of $Z$.

(These results summarize Proposition 2.1, 7.1 and 9.1 in [T2].)

Here is an important consequence of (1.4): If $p>0$ on a component of $Z$ and if $\tau \neq Z_{S}$ lies in that component, then $C$ near $\tau$ is described completely by the numbers $q_{ \pm}$plus a set $\left\{\varphi_{0 \alpha}\right\}_{1 \leq \alpha \leq p}$ of analytic functions which are defined on a neighborhood of $\tau$ in $Z$.

With the preceding understood, a definition of a boundary regular pseudoholomorphic subvariety is in order.

Definition 1.2. A pseudoholomorphic subvariety $C \subset X-Z$ is called boundary regular when it satisfies the following two constraints:

- $Z_{S}=\varnothing$.

- $q_{ \pm}=0$ along each component of $Z$.

Concerning this notion of boundary regularity, it is important to realize that (1.5) has the following consequence:

On some tubular neighborhood of each component of $Z$, a boundary regular, pseudoholomorphic subvariety $C$ is described by a non-negative integer $p$ and an unordered set of $\mathbb{R} /(2 \pi \mathbb{Z})$ valued, real analytic functions $\left\{\varphi_{0 \alpha}\right\}_{1 \leq \alpha \leq p}$ on $Z$

Indeed, start at a fixed point in the given component of $Z$, and then the local data from Part b of the third point (1.4) pieces together as the given component of $Z$ is circumnavigated to give an ordered set of real analytic functions. However, when the starting point is reached, the resulting ordered set of functions might differ from the original by a permutation of the indices $\{1, \cdots, p\}$. The components of $C$ near the given component of $Z$ are then in 1-1 correspondence with the set of orbits in $\{1, \cdots, p\}$ of the $\mathbb{Z}$ action generated by this permutation. If such an orbit has $p^{\prime}$ elements, then the corresponding component of $C$ can be parameterized by $S^{1} \times(0, \varepsilon)$ where the projection to the $(0, \varepsilon)$ factor is given by the restriction of the function $\rho$, and where the $S^{1}$ factor is parameterized by $\tau \equiv t / p^{\prime}$. Restriction of the functions $z$ and $\varphi$ to the given component of $C$ defined the latter as functions of $\rho$ and $\tau$, and then the part of $\mathrm{c}$ in question is parameterized via $(\tau, \rho) \rightarrow\left(p^{\prime} \tau, \rho \cos (\varphi(\tau, \rho)), \rho \sin (\varphi(\tau, \rho), z(\tau, \rho))\right.$.

Concerning these functions $z(\tau, \rho)$ and $\varphi(\tau, \rho)$ : Both are real analytic for $0 \leq \rho \leq \varepsilon$ for some positive $\varepsilon$; and both are determined by $\varphi_{0}(\tau) \equiv \varphi(\tau, 0)$ as in Part b of the third point of (1.4). Conversely, a positive integer $p^{\prime}$ and a real analytic map, $\varphi_{0}$, from $S^{1}$ to $S^{1}=\mathbb{R} /(2 \pi \mathbb{R})$ defines a boundary regular pseudoholomorphic subvariety in a small neighborhood of the given component of $Z$ via the power series expansion in $\rho$ which is implicit in Part b of the third point of (1.4).

The discussion of the past few paragraphs should have made clear that a boundary regular, pseudoholomorphic subvariety contains an open set with compact closure whose compliment is diffeomorphic as a manifold with boundary to a union of annuli of the form $S^{1} \times(0,1]$. Moreover, the completion of each such annulus using the induced metric from $X$ is $S^{1} \times[0,1]$. And, the tautological embedding of the half open annulus into $X$ extends to this closed annulus as a map into $X$ that sends $S^{1} \times\{0\}$ into some component of $Z$ as a covering map.

This section ends with some special examples of boundary regular, pseudoholomorphic subvarieties. These examples are defined in the neighborhood described in (1.1) of a component of $Z$. Also in these examples, the parameter $t$ in the component of $Z$ in question is normalized so as to identity the latter with $\mathbb{R} /(2 \pi \mathbb{Z})$. The 
examples below are characterized by an integer $p \geq 1$, a positive integer $q$ which is either zero, or relatively prime to $p$, and a number $\alpha \in \mathbb{R} /(2 \pi \mathbb{Z})$. With $(p, q, \alpha)$ given, the corresponding subvariety is parameterized by $(\tau, \rho) \in \mathbb{R} /(2 \pi \mathbb{Z}) \times[0,1]$ though the $\mathbb{R} /(2 \pi \mathbb{Z})$ valued function $\varphi(\tau, \rho)$ and the function $z(\tau, \rho)$. In this case, $\varphi \equiv q \tau+\alpha$ is a linear function of $\tau$, while $z$ depends only on $\rho$ and is the unique smooth function on $[0,1]$ which obeys

$$
\begin{aligned}
& \text { - }(p+2 q z) \rho \frac{d z}{d \rho}+2 p z-q \rho^{2}=0, \\
& \text { - } z(p)=\frac{q}{4 p} \rho^{2}+\mathcal{O}\left(\rho^{4}\right) .
\end{aligned}
$$

The following sketch illustrates one of the previous examples:

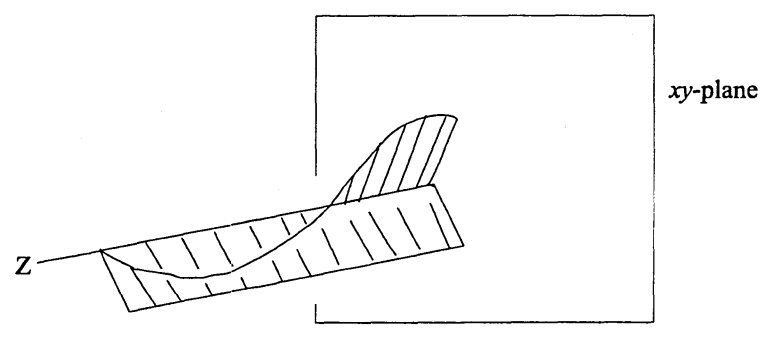

2. Moduli spaces for pseudoholomorphic subvarieties. This section provides the definition and describes some of the basic properties of the moduli space for the set of boundary regular pseudoholomorphic subvariaties. In addition, there is a final subsection which touches on a different topics, which is the observation that the numbers $q_{ \pm}$as defined in the last point in (1.4) are always zero if the metric and corresponding closed, self-dual 2-form, though subject to the constraints in (1.1), are chosen in a sufficiently generic way.

The proofs of the assertions in this section are deferred to the subsequent sections.

a) The moduli space. Let $\mathcal{M}$ denote the set of pseudoholmorphic subvarieties in $X-Z$. The definition below specifies a topology on $\mathcal{M}$.

Definition 2.1. Topologize $\mathcal{M}$ by defining a basis for the open neighborhoods of a given pseudoholomorphic subvariety $C$ as follows: The sets in this basis are labeled by the positive real numbers, and the set, $\mathcal{U}(\varepsilon)$, labeled by $\varepsilon>0$ consists of those $C^{\prime} \in \mathcal{M}$ for which

$$
\left|\int_{C} \omega-\int_{C^{\prime}} \omega\right|+\sup _{x \in C^{\prime}} \operatorname{dist}(x, C)+\sup _{x \in C} \operatorname{dist}\left(x, C^{\prime}\right)<\varepsilon
$$

The proposition below summarizes some of the elementary features of this topology:

Proposition 2.2. The assignment of $C \subset \mathcal{M}$ to the positive number $\int_{C} \omega \in$ $(0, \infty)$ defines a locally constant function on $\mathcal{M}$. In addition, each component of $Z$ defines, for each $C \in \mathcal{M}$, the numbers $q_{ \pm}$and $p$ as descibed in (1.4), and the combination $p-q_{+}-q_{-}$is a locally constant function on $\mathcal{M}$. In addition, the intersection number of $C \subset \mathcal{M}$ with any given oriented submanifold in $X-Z$ defines a locally constant function on $\mathcal{M}$ which depends only on the homology class of the given submanifold in $\mathrm{H}_{2}(X-Z ; \mathbb{Z})$. Finally, the area of $C \subset \mathcal{M}$ defines a continuous function on $\mathcal{M}$. 
b) The decomposition of the subspace of boundary regular subvarieties. Use $\mathcal{M}_{b} \subset \mathcal{M}$ to denote the subspace of boundary regular subvarieties. The Theorem from the introductory section refers to a stratification of $\mathcal{M}_{b}$ and the purpose of this subsection is to define the stratification. This is to say that the plan here is decompose $\mathcal{M}_{b}$ as a nested union of sets, $\left\{\mathcal{M}_{b, k}\right\}$ where $\mathcal{M}_{b}=\mathcal{M}_{b, 0}$ and where $\mathcal{M}_{b, k+1} \subset \mathcal{M}_{b, k+1}$ is closed. The following eight steps describe the criteria of membership in $\mathcal{M}_{b, k}$.

Step 1: Let $C \in \mathcal{M}_{b}$. Because $C$ is pseudoholomorphic, there is a smooth 2manifold with boundary, $\underline{C}_{0}$, with complex structure on its interior, $C_{0}$, and with a smooth map $\sigma: \underline{C}_{0} \rightarrow X$ which is almost everywhere 1-1, restricts to $C_{0}$ as a pseudoholomorphic map into $X-Z$ with image $C$, and restricts to $\partial \underline{C}_{0}$ as a map into $Z$ which is a local covering map onto its image. Let $\chi\left(C_{0}\right)$ denotes the Euler characteristic of $\underline{C}_{0}$.

Step 2: Introduce the pull-back $\sigma^{*} T X$. This bundle has an almost complex structure on $C_{0}$ which extends to one over $\underline{C}_{0}$. To define this extension, first observe that all of the singular points of $\sigma$ lie in $C_{0}$ (see (1.4)) and away from these points, $\sigma^{*} T X$ decomposes as $T C_{0} \oplus \sigma^{*} N$, where $N$ is the normal bundle to the image of $C_{0}$ where the latter is imbedded. The almost complex structure $\sigma^{*} T X$ respects this splitting, so the extension to $\partial \underline{C}_{0}$ of the almost complex structure on $T C_{0}$ and $\sigma N$ extend that on $\sigma^{*} T X$.

With the preceding understood, here is how to extend the almost complex structure on $T C_{0}$ : Because of Part b of the third point in (1.4), the functions $(t, \rho)$ from a neighborhood in $X$ of a component $Z^{\prime} \subset Z$ pull back by $\sigma$ to $\underline{C}_{0}$ as local coordinates near a boundary component which maps by $\sigma$ to $Z^{\prime}$. Here, $\rho=0$ on the boundary component in question, and the corresponding vector fields $\partial_{t}$ and $\partial_{\rho}$ are tangent to the boundary and inward pointing, respectively. And, on this component, the almost complex structure on $T \underline{C}_{0}$ sends $\partial_{t}$ to $-\partial_{\rho}$.

Meanwhile, the almost complex structure on $N$ extends over the same boundary component of $\underline{C}_{0}$ as follows: First, the normal bundle on the given boundary component is spanned by the vector field $\partial_{z}$ and $\cos \left(\varphi_{0}\right) \partial_{y}-\sin \left(\varphi_{0}\right) \partial_{x}$, where $\varphi_{0}$ is the restriction to $\partial \underline{C}_{0}$ of the $\mathbb{R} /(2 \pi \mathbb{Z})$ valued function $\varphi$. And, the almost complex structure on $N$ sends $\partial_{z}$ to $-\left(\cos \left(\varphi_{0}\right) \partial_{y}-\sin \left(\varphi_{0}\right) \partial_{x}\right)$.

With an almost complex structure on $\sigma^{*} T X$, the latter's complexification can be written as $V \oplus V^{*}$, where $V \rightarrow \underline{C}_{0}$ is the complex 2-plane bundle of type $(1,0)$ tangent vectors.

Step 3: The purpose of this step is to define a first Chern number for the complex line bundle $\Lambda^{2} V \rightarrow \underline{C}_{0}$. Such a number is defined with the specification of a nowhere vanishing section of $\Lambda^{2} V$ along $\partial \underline{C}_{0}$. Indeed, given the latter, this first Chern number is defined by first extending the given section over $C_{0}$ so its zeros are non-degenerate and then making the usual algebraic count of the zeros.

Meanwhile, a nowhere vanishing section of $\Lambda^{2} V$ along $\partial \underline{C}_{0}$ is defined by a pair consisting of nowhere vanishing sections of $T \underline{C}_{0}$ and of $N$ along $\partial \underline{C}_{0}$. Take the vector field $\partial_{t}$ as the section to use for $T \underline{C}_{0}$, and use the section of $N$ along $\partial \underline{C}_{0}$ which is defined in the next step.

Use the notation $c_{1}\left(\sigma^{*} T X\right)$ to denote the resulting first Chern number.

Step 4: This step constitutes a digression of sorts of define a nowhere vanishing section of $N$ along $\partial \underline{C}_{0}$. To begin, consider an end of $C$ where the $z$-axis line bundle is 
orientable. Pick a positive direction for the $z$-axis, and agree to push $C$ off itself along the given component of $Z$ by pushing into this positive $z$ direction. Pulled back to $\underline{C}_{0}$, this positive $z$-axis pushoff defines a section of $\sigma^{*} N$ near the relevant components of $\partial \underline{C}_{0}$ which extends over these boundary components as a nowhere vanishing section.

Near a component of $Z$ where the $z$-axis line bundle is not orientable, pick a starting point, 0 , on the given component of $Z$, and then pick a positive direction for the $z$-axis at 0 . Move in the positive direction along $Z$ and in doing so, push $C$ off of itself in the positive $z$ direction until a point just short of the return to 0 is reached. To obtain a continuous push-off, note that the normal bundle to $C$ near 0 is oriented, and so the original choice of $z$-axis at 0 defines a positive orthogonal axis in the normal bundle of $C$ near 0 . Use a push off $C$ near 0 into this positive orthogonal axis to connect the push-offs on either side of 0 . The following diagram illustrates:

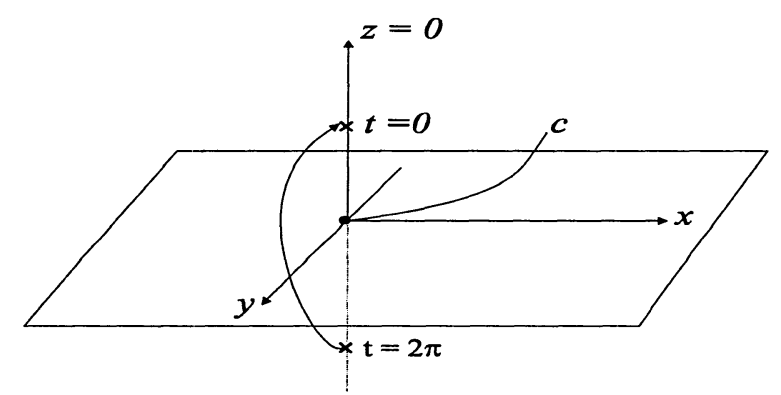

The push-off near 0 for $d_{c}$ in the non-orientable case

These push-offs of $C$ near $Z$ also pull back to $\underline{C}_{0}$ where they define a nowhere vanishing section of $\sigma^{*} N$ on the relevant components of $\partial \underline{C}_{0}$.

Step 5: The purpose of this step is to define a self intersection number of $C$. The latter will be denoted $C \bullet C$ and it is defined as follows: First, perturb $C$ near its singular points in $X-Z$ so that the result, $C^{\prime}$, agrees with $C$ near $Z$ and intersects $X-Z$ as a submanifold. Then, use the push-off of $C$ near $Z$ as described in the previous step to push $C^{\prime}$ off of itself near $Z$ and extend this push-off over the rest of $C^{\prime}$. The number $C \bullet C$ is then the intersection number of $C^{\prime}$ with such a push-off.

Step 6: With the preceding steps completed, consider:

Definition 2.3. For each integer $k \geq 0$, define $\mathcal{M}_{b, k} \subset \mathcal{M}_{b}$ to be the subspace of $C \in \mathcal{M}_{b}$ for which

$$
k \leq k(C) \equiv C \bullet C-c_{1}\left(\sigma^{*} T X\right)+\chi\left(C_{0}\right) .
$$

In the event that $C$ is immersed, the formula for $k(C)$ can be expressed in somewhat more enlightening fashion in terms of a pair of integers which characterize, in a sense, the number of double points of the extension of $\sigma$ to the manifold with boundary $\underline{C}_{0}$. The first such integer, $m_{C}$, is defined to be the number of double points of $C \subset X-Z$ as defined in the usual way. That is, $m_{C}$ is the number of distinct, unordered, 2-element subsets $\left\{o, o^{\prime}\right\} \subset C_{0}$ with the property that $\sigma(o)=\sigma\left(o^{\prime}\right)$. The second integer, $n_{C}$, is a sort of measure of the degree to which the extended $\sigma$ fails to immerse $\partial \underline{C}_{0}$. To be precise, $n_{C}$ is obtained as a sum whose terms are indexed 
by data of the form $\left(\tau,\left\{\varphi_{0 \alpha^{\prime}}, \varphi_{0 \alpha^{\prime \prime}}\right\}\right)$ where $\tau \in Z$ and where $\left\{\varphi_{0 \alpha^{\prime}}, \varphi_{0 \alpha^{\prime \prime}}\right\}$ is an unordered, 2-element subset from (1.5)'s data $\left\{\varphi_{0 \alpha}\right\}$ whose values coincide at $\tau$. The contribution to $n_{C}$ from such a data set is defined to be the degree of vanishing at $\tau$ of $\varphi_{0 \alpha^{\prime}}-\varphi_{0 \alpha^{\prime \prime}}$.

With $m_{C}$ and $n_{C}$ understood, it then follows from Lemma 4.1, below, that

$$
c_{1}\left(\sigma^{*} T X\right)=C \bullet C+\chi\left(C_{0}\right)-2 m_{C}-n_{C} .
$$

Thus, if $C \in \mathcal{M}_{b}$ is immersed, then

$$
k(C)=2 m_{C}+n_{C} .
$$

In particular, note that when $C$ is embedded and when the integer $p$ from (1.5) is one along each component of $Z$, then $k(C)=0$ and so $C$ is in the top strata of $\mathcal{M}_{b}$.

c) The properties of $\left\{\mathcal{M}_{b, k}\right\}_{k \geq 0}$. The purpose of this subsection is to describe some of the salient features of the $\left\{\mathcal{M}_{b, k}\right\}$. In particular, note that the Theorem from the Introduction is a direct consequence of Proposition 2.4, 2.5 and 2.6.

Proposition 2.4. The subset $\mathcal{M}_{b}$ is an open subset of $\mathcal{M}$.

Proposition 2.5. Define $\left\{\mathcal{M}_{b, k}\right\}_{k \geq 0}$ as above. Then $\mathcal{M}_{b}=\mathcal{M}_{b, 0}$ and for $k \geq 0$, $\mathcal{M}_{b, k+1}$ is a closed subset of $\mathcal{M}_{b, k}$.

Proposition 2.6. Suppose that $k \geq 0$ and that $C \subset \mathcal{M}_{b, k}-\mathcal{M}_{b, k+1}$. Then $C$ has a neighborhood $\mathcal{U} \subset \mathcal{M}_{b, k}$ with the following property: There exists a Fredholm operator $D_{C}$, a ball $B \subset$ kernel $\left(D_{C}\right)$, a smooth map $f: B \rightarrow$ cokernel $\left(D_{C}\right)$ which takes 0 to 0 , and a homeomorphism $\mathcal{F}$ from $f^{-1}(0)$ onto $\mathcal{U}$.

The next proposition describes (in part) how the data $\left\{\varphi_{0 \alpha}\right\}$ from (1.5) which describes a boundary regular subvariety near $Z$ varies with the chosen variety in $\mathcal{M}_{b}$. In this regard, remember that the data $\left\{\varphi_{0 \alpha}\right\}$ can be viewed as an ordered set of real analytic maps from $S^{1}$ to $\mathbb{R} /(2 \pi \mathbb{Z})$, where each $\varphi_{0}$ in the latter set corresponds to an end of $C$ near the given component of $Z$. Here, the map $\varphi_{0}$ which corresponds to a given end of $C$ is the $\rho=0$ limit of the restriction to said end of the function $\varphi$.

Proposition 2.7. Suppose that $k \geq 0$ and that $C \subset \mathcal{M}_{b, k}-\mathcal{M}_{b, k+1}$. Then $C$ has an open neighborhood $\mathcal{U} \subset \mathcal{M}_{b, k}-\mathcal{M}_{b, k+1}$ with the following property: Let $Z^{\prime} \subset Z$ be a component. The ends near $Z^{\prime}$ of any $C^{\prime} \in \mathcal{U}$ can be put in 1-1 correspondence with those of $C$. And, specify an end of $C$ and thus a corresponding end of any element in $\mathcal{U}$. The latter is defined (as described in (1.5)) by a positive integer $p^{\prime}$ and a real analytic map $\varphi_{0}$ from $S^{1}$ to $S^{1}=\mathbb{R} /(2 \pi \mathbb{Z})$. This integer $p^{\prime}$ is the same for all element of $\mathcal{U}$; while the association of the map $\varphi_{0}$ to an element in $\mathcal{U}$ defines a continuous map from $\mathcal{U}$ into the Frechet space of real analytic maps from $S^{1}$ to $S^{1}$.

d) The operator $D_{C}$. The operator $D_{C}$ which appears in Proposition 2.6 is obtained from a certain $\bar{\partial}$ operator on the smooth model $\underline{C}_{0}$ with an added zero'th order, $\mathbb{R}$-linear term. If $C$ is immersed, then the relevant $\bar{\partial}$ operator acts on sections of $C$ 's normal bundle. In the non-immersed case, the $\bar{\partial}$ operator in question acts on sections of the bundle $V$ from Step 2 of Section $2 \mathrm{~b}$. (On the interior, $C_{0}$ of $\underline{C}_{0}$, the bundle $V$ is the pull-back via $\sigma$ of the bundle of type $(1,0)$ complex tangent vectors of $X-Z$.) In either case, the domain and range of $D_{C}$ are obtained by completing, with a norm of Sobolev type, a space of sections of the relevant vector bundle. This is analogous to the procedure followed when $C$ is compact and $X$ is symplectic. However, 
there is one substantive difference, which is that the sections which define the domain are constrained appropriately on $\partial \underline{C}_{0}$. The details of this are fairly technical and the reader is referred to Section 5 , below, for the rest of the story.

The formal dimension of $\mathcal{M}_{b, k}$ in Proposition 2.5 is given by the index of the operator $D_{C}$. The next proposition gives a geometric formula for this index. This geometric data consists of the Euler characteristic, $\chi\left(C_{0}\right)$, the characteristic number $c_{1}\left(\sigma^{*} T X\right)$ from Step 3 of Section $2 \mathrm{~b}$, and a non-negative number $N^{\prime}(C)$. The definition of $N^{\prime}(C)$ requires a short digression: To begin the definition, remember that the $z$-axis, as defined locally in (1.1), may or may not define an orientable line bundle near any given component of $Z$. In any event, each end of $C$ inherits, by restriction, the $z$-axis line bundle from $Z$, and on each end of $C$, this line bundle can be either orientable or not. Given the preceding, the number $N^{\prime}(C)$ is the number of ends of $C$ to which the restriction of the $z$-axis line bundle is not orientable.

With the digression now over and $N^{\prime}(C)$ defined, consider

Proposition 2.8. The index of Proposition 2.6's operator $D_{C}$ is

$$
\text { index }\left(D_{C}\right)=2 c_{1}\left(\sigma^{*} T X\right)-\chi\left(C_{0}\right)+N^{\prime}(C) .
$$

Note that when $C$ is immersed, then (2.3) can be used to write (2.5) in ways which may or may not be more appealing.

What follows are two examples of the formula in (2.5). For the first example, let $M$ be a compact, oriented, 3-manifold with $b^{1}>0$ and take $X=S^{1} \times M$. If the metric on $X$ is suitably generic, a non-zero class in $H^{1}(M ; \mathbb{Z})$ is represented by a harmonic map $\varphi: M \rightarrow S^{1}$ with non-degenerate critical points. (See, e.g. [Ho].) Moreover, one can find such a metric and coordinates $(x, y, z)$ on some neighborhood of each of $\varphi$ 's critical points which make the metric the standard Euclidean one and which put $v \equiv-i \varphi^{-1} d \varphi$ into the standard form: $x d x+y d y-2 z d z$. Now take the product metric on $X$. Then, introduce $\omega \equiv d t \wedge v+* v$, where $t$ is a standard affine coordinate on $S^{1}$ and where $*$ denotes the Hodge star on $M$. This 2 -form is closed and self dual. Moreover, it vanishes transversally in a union of circles and (1.1) is satisfied near each such circle. (The circles in question are the products of $S^{1}$ with the critical points of $v$.)

Now, let $\gamma \subset M$ be a noncompact gradient flow line for the dual vector field to $v$ which lies in the intersection of the ascending disk of an index 1 critical point and the descending disk of an index 2 critical point. Then, $C \equiv S^{1} \times \gamma$ is a boundary regular, pseudoholomorphic submanifold in $X$. To compute the index of $D_{C}$, note the each component of $Z$ has oriented $z$-axis, and thus $N^{\prime}(C)=0$ in Proposition 2.8. Also, $\chi\left(C_{0}\right)=0$ since $C$ is a cylinder. It is left to the reader to check that $C \bullet C=0$ as well, and so according to (2.3) and (2.5), $D_{C}$ has index 0 . (It is an exercise to identify the kernel and cokernal of $D_{C}$ purely in terms of an operator on sections over $\gamma$ of its normal bundle in $M$.)

Here is the second example (the idea for this example came from work of Luttinger $[\mathrm{Lu}])$ : To begin, take $X=\mathbb{R}^{4} \equiv \mathbb{C}^{2}$ with complex coordinates $(\lambda, \eta)$. Use the standard Euclidean metric on $\mathbb{R}^{4}$. Let $\omega$ denote the following self dual, harmonic 2 -form on $\mathbb{R}^{4}$ :

$$
\begin{aligned}
\omega \equiv i 2^{-1}\left[\left(1-|\lambda|^{2}+|\eta|^{2}\right)(d \lambda \wedge d \bar{\lambda}+d \eta \wedge d \bar{\eta})\right. \\
+(\bar{\lambda} \bar{\eta}-R \eta) d \lambda \wedge d \eta-(\lambda \eta-R \bar{\eta}) d \bar{\lambda} \wedge d \bar{\eta}] .
\end{aligned}
$$

Here, $R \geq 0$ is a constant. 
When $R>1$ in (2.6), the zero set of $\omega$ consists of the circle, $Z^{\prime}$, where $|\lambda|=1$ and $\eta=0$ and another circle with $|\lambda|=R$. Of interest is the former component, $Z^{\prime}$. This example obeys (1.1) only to leading order in the distance to $Z^{\prime}$ so there is still a well defined $z$-axis bundle over $Z^{\prime}$. The latter is non-orientable. Also, the disk $C=\{(\lambda, \eta):|\lambda|<1$ and $\eta=0\}$ is pseudoholomorphic and its only end approaches $Z^{\prime}$ with $p=1$.

Note that this example does not obey (1.1). However, it is a straightforward task to perturb this example in any chosen neighborhood of $Z^{\prime}$ to obtain a new metric and self dual, harmonic 2-form which does conform to (1.1). In fact, given $\varepsilon>0$, the new form and metric can be taken to be $\varepsilon$ close in the $C^{1}$ topology to the old ones. Furthermore, the perturbation can be made so that the disk $C$ stays pseudoholomorphic. As this perturbed version obeys (1.1), Proposition 2.8 can be employed to compute the index of the resulting operator $D_{C}$. To make the story short, the value of the index (as computed using (2.3) in (2.5)) is zero because $\chi(C)=1$, $N^{\prime}(C)=1$ and $C \bullet C=-1$. (The computation of $C \bullet C$ is test of endurance to get all of the orientations correct.)

One can also consider (2.6) with $0 \leq R<1$. In this case, there is just one component of $Z$, the radius 1 circle in the $\eta=0$ plane. For these values of $R$, the $z$-axis line bundle here is orientable. Once again, the disk $C=\{(\lambda, \eta):|\lambda|<1$ and $\eta=0\}$ is pseudoholomorphic, but now its only end approaches $Z^{\prime}$ with $p=0$ with $q=1$. Thus, the disk in this case is not in $\mathcal{M}_{b}$ and Proposition 2.8 has nothing to say. However, this case is discussed further in Subsection $2 \mathrm{f}$ below.

e) Additional properties of $\mathcal{M}_{b}$ related to behavior near $Z$. As previously noted in (1.5), the intersection of a given $C \in \mathcal{M}_{b}$ with an appropriate tubular neighborhood of any given component $Z^{\prime} \subset Z$ is characterized by an integer $p \geq 0$ and a set $\left\{\varphi_{0 \alpha}\right\}_{1 \leq \alpha \leq p}$ of locally defined, real analytic, $\mathbb{R} /(2 \pi \mathbb{Z})$-valued functions on $Z^{\prime}$. The set can be ordered unambiguously on any open interval in $Z^{\prime}$, but any ordering suffers a permutation upon circumnavigation of $Z^{\prime}$. Here is another way to say the same thing: The restriction of the function $\varphi$ to the $\rho=$ constant slices of $C$ near $Z^{\prime}$ defines, in the limit as $\rho$ tends to zero, a real analytic map from a $p$-fold cover of $Z^{\prime}$ to $\mathbb{R} /(2 \pi \mathbb{Z})$ whose push-forward produces the set $\left\{\varphi_{0 \alpha}\right\}$. Meanwhile, the component of this $p$-fold cover of $Z^{\prime}$ are in 1-1 correspondence with the components of $C$ near $Z^{\prime}$.

As $C$ near $Z^{\prime}$ is a union of annuli, and as $\varphi$ maps the latter into $\mathbb{R} /(2 \pi \mathbb{Z})$, there is well defined, integer valued degree which $Z^{\prime}$ associates to $C$. Note that this degree is not the same as the covering degree $p$. In the example of (1.6), the degree in question is equal to the integer $q$.

If the reader is uneasy with the absence of a global ordering for the set $\left\{\varphi_{0 \alpha}\right\}$, view the latter, at the loss of some information, as the data for a map, $\Phi$, from $Z^{\prime}$ into the $p^{\prime}$ th symmetric product of the circle. In fact, identity $\operatorname{Sym}^{p} S^{1}$ with the obvious subset of $\operatorname{Sym}^{p} \mathbb{C}$ and identify $\operatorname{Sym}^{p} \mathbb{C}$ with $\mathbb{C}^{p}$ via the map which send the unordered set $\left\{\lambda_{1}, \cdots, \lambda_{p}\right\}$ of complex numbers to the ordered set of coefficients $\left(a_{1}, \cdots, a_{p}\right)$ of the polynomial $z^{p}+a_{1} z^{p-1}+\cdots a_{P} \equiv \Pi_{1 \leq \alpha \leq p}\left(z-\lambda_{\alpha}\right)$. Then, the map $\Phi$ from $Z^{\prime}$ to $\mathbb{C}^{p}$ is real analytic.

Move to a different, but nearby point in the moduli space $\mathcal{M}_{b}$, and the data $\left\{\varphi_{0 \alpha}\right\}$ must change. If the new point is in the same (open) stratum, $\mathcal{M}_{b, k}-\mathcal{M}_{b, k+1}$, as the old, then Proposition 2.7 details the change in $\left\{\varphi_{0, \alpha}\right\}$. If the new point lies in a different stratum, the following proposition describes (in part) how $\left\{\varphi_{0 \alpha}\right\}$ changes. 
Proposition 2.9. The multiplicity, $p$, associated by each component of $Z$ to a boundary regular subvariety defines a locally constant function on $\mathcal{M}_{b}$. Likewise, the degree of the map $\varphi$ that each component of $Z$ associates to a regular, pseudoholomorphic subvariety is also locally constant on $\mathcal{M}_{b}$. Furthermore, specify a component $Z^{\prime} \subset Z$ and the assignment of the corresponding data $\left\{\varphi_{0 \alpha}\right\}_{1 \leq \alpha \leq p}$ to an element of $\mathcal{M}_{b}$ defines a continuous map from $\mathcal{M}_{b}$ into the Banach space of continuous maps of $Z^{\prime}$ into $\operatorname{Sym}^{p}(\mathbb{C})$.

Given a component $Z^{\prime} \subset Z$, the number of ends near $Z^{\prime}$ of a subvariety from $\mathcal{M}_{b}$ does not define a continuous function. However, such is the case on a subspace $\mathcal{M}_{r b} \subset \mathcal{M}_{b}$ where the following condition is enforced:

For each $\tau \subset Z$, introduce (1.5)'s set $\left\{\varphi_{0 \alpha}\right\}_{1 \leq \alpha \leq p}$ of analytic functions which are defined on $Z$ near $\tau$ and describe $C$ near $\tau$. If a distinct pair of functions from the set $\left\{\varphi_{0 \alpha}\right\}_{1 \leq \alpha \leq p}$ coincide at $\tau$, then the pair have distinct derivatives at $\tau$.

The following proposition concerns the ends of subvarieties in $\mathcal{M}_{r b}$ :

Proposition 2.10. The subspace $\mathcal{M}_{r b}$ is an open subsets of $\mathcal{M}$. Moreover, if $C \subset \mathcal{M}_{r b}$, then $C$ has a neighborhood $\mathcal{U}$, with the following properties: Let $Z^{\prime} \subset Z$ be a component. The ends near $Z^{\prime}$ of any $C^{\prime} \in \mathcal{U}$ can be put in 1-1 correspondence with those of $C$. In addition, specify an end of $C$ and thus a corresponding end of any element in $\mathcal{U}$. The latter is defined (as described above) by a positive integer $p^{\prime}$ and a real analytic map $\varphi_{0}$ from $S^{1}$ to $S^{1}=\mathbb{R} /(2 \pi \mathbb{Z})$. Then, this integer $p^{\prime}$ is the same for all elements of $\mathcal{U}$; while the association of the map $\varphi_{0}$ to an element in $\mathcal{U}$ defines a continuous map from $\mathcal{U}$ into the Frechet space of real analytic maps form $S^{1}$ to $S^{1}$.

There almost surely exist pseudoholomorphic submanifolds $C \in \mathcal{M}_{b}-\mathcal{M}_{r b}$ which have neighbors in $\mathcal{M}_{b}$ having fewer ends, but an explicit example is not available.

The discussion in Section 2 of $\mathcal{M}_{b}$ is now at its end.

f) The appearance of $q_{ \pm}$. This last subsection considers a topic which is somewhat disjoint from the previous topics as it describes the subset of pairs of Riemannian metrics on $X$ and corresponding self dual 2-forms for which (1.1) is obeyed and which have the following additional property: Every $C \in \mathcal{M}$ has $q_{ \pm}=0$ along each component of $Z$. The description of this set is provided by the next two propositions.

Proposition 2.11. Let $\mathcal{G}$ denote the set of pairs consisting of a smooth metric on $X$ and a closed, self-dual 2-form which together obey (1.1). There is a subset $\mathcal{G}^{\prime} \subset \mathcal{G}$ which is a countable intersection of open and dense sets (for the $C^{\infty}$ topology) and which is characterized by the following property: Let $C \subset X-Z$ be any pseudoholomorphic subvariety as defined using the chosen metric and closed, self-dual 2-form from $\mathcal{G}^{\prime}$. Then both $q_{ \pm}$from (1.4) vanish at each point of $Z$.

The following proposition details an additional property of $\mathcal{G}^{\prime}$ :

Proposition 2.12. The inclusion $\mathcal{G}^{\prime} \subset \mathcal{G}$ induces homotopy equivalence.

These last two propositions are proved in Section 6. Suffice it to say for now that the deformation problem for a pseudo-holomorphic subvariety with $q_{ \pm} \neq 0$ does not even have deformations which are local to $Z$. (One can view this as a consequence of the fact that the first order deformation problem is described by an operator with 
infinite dimensional cokernel. In this regards, think of this cokernel as the obstruction to keeping the subvariety through a deformation of the almost complex structure.)

The $R=0$ case of the form in (2.6) provides a very explicit example. As remarked, $Z$ in this case consists of the standard unit circle in the $\eta=0$ plane. Also, the standard unit disk in the $\eta=0$ plane is pseudoholomorphic. But, this disk has $p=0$ and $q=1$. A deformation of this disk can be described by giving $\eta$ as a function of the complex coordinate $\lambda$ on the disk. In particular, the first order deformations of this disk correspond to functions $\eta$ on the disk which obey the following equation:

$$
\partial_{\lambda} \eta-\frac{1}{2} \frac{\bar{\lambda}}{\left(1-|\lambda|^{2}\right)} \eta=0 .
$$

The solutions to this equation all have the form

$$
\eta=\frac{\eta_{0}(\bar{\lambda})}{\sqrt{\left(1-|\lambda|^{2}\right)}},
$$

where $\eta_{0}$ is an anti-holomorphic function of $\lambda$.

Note that (2.9) holds on any open subset of the disk, and in particular, an annulus with outer boundary the $|\lambda|=1$ circle. As there are no non-trivial anti-holomorphic functions on such an annulus which vanish on the $|\lambda|=1$ circle, there are no locally defined pseudo-holomorphic perturbations of the disk.

g) Table of Contents. The remainder of this article is occupied with the proofs of the propositions in this section. Here is a directory:

$\S 3$. Proofs of Propositions 2.2, 2.4, 2.9 and 2.10

$\S 4$. Proofs of Propositions 2.5 and 2.7 .

$\S 5$. Proofs of Propositions 2.6 and 2.8 .

$\S 6$. Proofs of Propositions 2.11 and 2.12 .

3. Convergence in $\mathcal{M}$. This section contains the proofs of Proposition 2.2 (in Section 3c), Proposition 2.4 (in Section 3d), Proposition 2.9 (in Sections 3e and 3f) and Proposition 2.10 (in Section $3 \mathrm{~h}$ ). The section also contains a more general discussion of the notion of convergence in $\mathcal{M}$.

a) Geometric convergence. This subsection consists of a digression whose purpose is to introduce the notion, from Definition 3.1 of [T2], of geometric convergence for sequence of pseudoholomorphic subvarieties. This notion is reproduced in

DeFinition 3.1. Let $Y$ be a (possibly non-compact) 4-manifold with symplectic form $\omega$ and with a compatible almost complex structure $J$. Let $\Omega$ be a countable, decreasing sequence of positive numbers with limit zero and let $\left\{C_{s}\right\}_{s \in \Omega} \in Y$ be a corresponding sequence of finite energy, pseudoholomorphic subvarieties. Let $C \subset Y$ be a finite energy, pseudoholomorphic subvariety as well. Say that $\left\{C_{s}\right\}$ converges geometrically to $C$ when the following two requirements are met:

- Treat each $C_{s}$ and also each irreducible component of $C$ as a 2-dimensional, rectifiable current which assigns to a smooth 2-form with compact support in $Y$ the integral of the form over the subvariety in question. Let $\mathcal{C}$ denote the set of irreducible components of $C$. Require that the sequence of currents $\left\{C_{s}\right\}$ converge weakly as a current to the current $\sum_{C^{\prime} \in \mathcal{C}} m_{C^{\prime}} C^{\prime}$, where $m_{C^{\prime}}$ is, for each $C^{\prime} \in \mathcal{C}$, a positive integer. 
- For each compact subset $K \subset Y$, introduce the number, $d_{K}(s)$, which is the supremum over pairs $(x, y) \in\left(C_{s} \cap K\right) \times(C \cap K)$ of the sum of the distances from $x$ to $C$ and from $y$ to $C_{s}$. Then, for each such $K$, require that the limits as $s \rightarrow 0$ of the numbers $d_{K}(s)$ exist and equal zero.

This notion of geometric convergence is observably at least as strong as the notion of convergence in $\mathcal{M}$. The fact that they are equivalent notions is asserted as

Lemma 3.2. A sequence $\left\{C_{i}\right\} \subset \mathcal{M}$ which converges to $C \in \mathcal{M}$ converge geometrically to $C$.

The proof of this lemma requires the following auxiliary lemma:

Lemma 3.3. Let $\left\{C_{i}\right\} \subset \mathcal{M}$ converge to $C \in \mathcal{M}$. Then the sequence of numbers whose $i$ 'th term is the area of $C_{i}$ is bounded and converges to the area of $C$.

Proof of Lemma 3.3. This is an immediate consequence of Proposition 2.1 in [T2].

With the preceding lemma in place, consider the

Proof of Lemma 3.2. As the areas of the $C_{i}$ 's are uniformly bounded, the sequence $\left\{C_{i}\right\}$ is norm bounded when viewed as a sequence of 2-dimensional, rectifiable currents. Indeed, if $C^{\prime} \subset\left\{C_{i}\right\}$ and if $\kappa$ is a smooth 2 -form, the $\left|\int_{C^{\prime}} \kappa\right| \leq \zeta \cdot \sup _{X}|\kappa|$.

As a bounded sequence of rectifiable currents, any subsequence of $\left\{C_{i}\right\}$ will have, according to either Proposition 3.3 or 3.8 in [T2], a subsequence which converges geometrically. Choose such a geometrically convergent subsequence and let $\mathcal{C}$ denote the set which defines the limit. Since $\left\{C_{i}\right\}$ converges pointwise to $C$, it follows that the subvarieties which appear in the set $\mathcal{C}$ consist precisely of the irreducible components of $C$. Furthermore, $\mathcal{C}$ associates a positive multiplicity to each such component, but each such multiplicity must equal one. Indeed, this last assertion follows from the assumed convergence to the energy of $C$ of the sequence whose $i$ 'th term is the energy of $C_{i}$. Since all multiplicities are one, the preceding argument demonstrates that the given subsequence of $\left\{C_{i}\right\}$ must converge geometrically to $C$. This last point implies that convergence in $\mathcal{M}$ implies geometric convergence to the same limit.

b) Convergence away from $Z$. This subsection analyzes the convergence of $C_{i}$ to $C$ near points of $C$ which are uniformly far from $Z$. The following lemma summarizes the conclusions.

Lemma 3.4. Let $p \in C$ be a manifold point. There exist a disk $D \subset C$ centered at $p, a$ disk $D^{\prime} \subset \mathbb{C}$ centered at the origin and an embedding $e: D \times D^{\prime} \rightarrow X$ which sends $D \times 0$ to $D$ and which has the following property with respect to the sequence $\left\{C_{i}\right\}$ : For all $i$ sufficiently large, the e-inverse image of $C_{i} \cap e\left(D \times D^{\prime}\right)$ is the graph of a smooth map $\eta_{i}: D \rightarrow D^{\prime}$. Moreover, the sequence $\left\{\eta_{i}\right\}$ converges to zero in the $C^{\infty}$ topology as $i$ tends to infinity.

Proof of Lemma 3.4. As indicated in Lemma 5.4 of [T5], one can choose disks $D_{1} \subset C$ centered at $p, D^{\prime} \subset \mathbb{C}$ centered at 0 and an embedding $e: D_{1} \times D^{\prime} \rightarrow$ $X$ which maps $D_{1} \times 0$ to $D_{1}$ and is such that the submanifold $e\left(w \times D^{\prime}\right) \subset X$ is pseudoholomorphic for each $w \in D_{1}$. Since $\left\{C_{i}\right\}$ converges geometrically to $C$, it follows that when $i$ is large, each $C_{i}$ has algebraic and hence geometric intersection number 1 with each such pseudoholomorphic disk. Thus, when $i$ is large, $C_{i} \cap$ Image $(e)$ is the image via $e$ of the graph of a continuous map $\eta_{i}: D_{1} \rightarrow D_{i}$. Moreover, the fact that $C_{i}$ is pseudoholomorphic translates into an elliptic, first order differential equation for $\eta_{i}$. Since $\left\{\eta_{i}\right\}$ converges pointwise to zero uniformly over $D_{1}$, standard 
elliptic estimates can be employed to prove that $\left\{\eta_{i}\right\}$ converges to zero in the $C^{\infty}$ topology on any concentric disk $D \subset D_{1}$ of strictly smaller radius.

c) Proof of Proposition 2.2. With Lemmas 3.2-3.4 in hand, the proof of Proposition 2.2 can be completed with the following argument. To begin, let $\mu$ be any 2 -form on $X$ with compact support in $X-Z$. Lemma 3.2 implies that integration of $\mu$ defines a continuous function on $\mathcal{M}$. This implies that the intersection numbers with compact submanifolds in $X-Z$ define locally constant functions on $\mathcal{M}$.

The fact that the assignment of $p-q_{+}-q_{-}$(as defined by a component of $Z$ ) to each $C \in \mathcal{M}$ defines a locally constant function follows from the observation that the latter is the intersection number of $C \in \mathcal{M}$ with a generic linking 2-sphere of the component of $Z$ in question.

The continuity of the area as a function on $\mathcal{M}$ is stated and proved as part of Lemma 3.3.

Finally, consider the function on $\mathcal{M}$ that is defined by integration of $\omega$. The latter is continuous (by definition) and so at issue here is whether it is a locally constant function. To see that such is the case, first fix a cut-off function $\chi:[0, \infty) \rightarrow[0,1]$ which is non-increasing, 1 on $[0,1]$ and 0 on $[2, \infty)$. Next, observe that $\omega$ is exact near $Z$. In fact, if follows from (1.3) that near any given component of $Z, \omega=d a$, where $a=-f d t-h d \varphi$ and so has norm $|a| \leq \zeta|\omega|^{2}$.

With all of this understood, fix $\varepsilon>0$ but tiny and note that $\omega_{\varepsilon}=\omega-d(\chi(|\omega| / \varepsilon) a)$ is cohomologous to $\omega$. Furthermore, a crude integration by parts argument shows that integration of $\omega_{\varepsilon}$ over $C \in \mathcal{M}$ gives the same as integration of $\omega$ over $C$. Indeed, to prove this claim, consider, for $s \in(0, \varepsilon)$, the integral over $C$ of $(1-\chi(|\omega| / s))\left(\omega-\omega_{\varepsilon}\right)$. Integration by parts and the observed bound on $|a|$ finds the latter integral no greater than $\zeta s$ area $(C)$. Now take $s$ to zero to obtain the claim.

To continue the argument for the local constancy of the integration of $\omega$ function, suppose now that $C \in \mathcal{M}$ and that $\left\{C_{i}\right\} \subset \mathcal{M}$ converges to $C$. Given $\varepsilon>0$, Sard's theorem finds some $\varepsilon^{\prime} \in(0, \varepsilon / 4)$ for which the $|\omega|=\varepsilon^{\prime}$ locus misses all non-manifold points of $C$. Moreover, Sard's theorem finds such $\varepsilon^{\prime}$ which is a regular value of the restriction of $|\omega|$ to $C$. With $\varepsilon^{\prime}$ fixed, Lemma 3.4 implies that these same two conclusions hold for each $C_{i}$ if $i$ is sufficiently large. Furthermore, the convergence of $C_{i}$ to $C$ as detailed in Lemma 3.4 implies that for $i$ large, a closed, null-homologous cycle in the $|\omega|>\varepsilon^{\prime}$ portion of $X-Z$ is obtained by taking the union of $C$ with the orientation reversed version of $C_{i}$ and then joining their boundaries on the $|\omega|=\varepsilon^{\prime}$ locus by the obvious linear deformation in the normal bundle of $C$. (Note that this cycle lives entirely in a tubular neighborhood of $C$.) Use $L_{i}$ to denote this closed cycle.

Since $L_{i}$ is null-homologous, $\omega_{\varepsilon}$ has integral zero over $L_{i}$. On the otherhand, $\omega_{\varepsilon}=0$ where $L_{i}$ is not either $C$ or $C_{i}$, and so the integral of $\omega_{\varepsilon}$ over $C$ must be equal to its integral over $C_{i}$ at least when $i$ is large. Thus, the integrals of $\omega$ over $C$ and over $C_{i}$ must also agree when $i$ is large.

d) Proof of Proposition 2.4. To start the argument, suppose here that $C \subset$ $\mathcal{M}_{b}$ and that $\left\{C_{i}\right\} \subset \mathcal{M}$ converges to $C$. The proof that $\mathcal{M}_{b}$ is open is obtained with a demonstration that $C_{i} \in \mathcal{M}_{b}$ when $i$ is large.

The analysis of the convergence of $\left\{C_{i}\right\}$ near $Z$ invokes certain properties of $C$ and the discussion starts by summarizing them. In particular, note that because of (1.4), there exists $\varepsilon>0$ and $\zeta \geq 1$ such that each point in $C$ which lies in the tubular neighborhood of a component, $Z^{\prime}$, of $Z$ from (1.1) and which has $r \leq \varepsilon$ also obeys $|z|<\zeta \rho^{2}<\rho / 200$. By choosing $\varepsilon$ smaller if necessary, one can assume that the 
$r \leq \varepsilon$ part of $C$ intersects each ball of radius $\varepsilon$ centered on $Z^{\prime}$ as a union of some fixed number, $p$, components, where the closure of each component is an embedded half disk which can be parameterized as in the third point of (1.4).

To simplify the proof's notation, agree henceforth that $C$ denotes only the $r \leq \varepsilon$ part of the variety in the previous two paragraphs.

Now, for $s \in(0, \varepsilon)$, consider a ball $B \subset X$ of radius $s$ and center on $Z^{\prime}$. It then follows directly form (1.4) that

$$
\int_{C \cap B} \omega=2 p s^{3} / 3+\mathcal{O}\left(s^{4}\right)
$$

Since there is an apriori bound on the area of each $C_{i}$, it follows from Lemma 3.4 that with $s$ fixed, and $\delta>0$ chosen, there exists $I=I(s, \delta)$ such that when $i>I$, then the integral of $\omega$ over $C_{i} \cap B$ is within $2 \delta s^{3} / 3$ from $2 p s^{3} / 3$. Remember this bound.

Now the reader is urged to read Section 4 of [T2] and especially Proposition 4.1 of [T2]. With Proposition 4.1 in [T2] understood, suppose that the index $i$ is large and that $C_{i}$ is not in $\mathcal{M}_{b}$. Fix $\tau \in Z$ and then rescale coordinates centered at $\tau$ as in Section 4 of [T2] to define a 2-dimenisonal current, $\mathcal{C}$, on $\mathbb{R} \times \mathbb{R}^{3}$ as described in Proposition 4.1 and Equation 4.1 of [T2]. This current gives the 'tangent cone' of $C_{i}$ at $\tau$. Since $C_{i}$ is assumed to lie in $\mathcal{M}-\mathcal{M}_{b}$, there will be a component $Z^{\prime}$, and a point $\tau \in Z^{\prime}$ where at the least one of the following assertions holds:

- Counting multiplicities, $\mathcal{C}$ contains at least $p+1$ submanifolds on which $z=0$ and $\varphi$ is constant. In this case, there will be at least one submanifold in $\mathcal{C}$ which coincides with the $t$ - $z$ plane.

- Counting multiplicities, $\mathcal{C}$ contained at least $p$ submanifolds on which $z=0$ and $\varphi=$ constant, and at least one cone on which $(\varphi, h)$ restrict without critical points. On each such cone, the integral of $d \varphi$ over a level set of $h$ is a positive integral multiple of $2 \pi$.

(Note that $\mathcal{C}$ contains at least $p$ submanifolds on which $z=0$ and $\varphi$ is constant because $C_{i}$ has intersection number $p$ with each linking 2 -sphere of the component $Z^{\prime}$.)

If follows from (3.2) that given $i$, there exists $s_{i}>0$ such that when $s^{\prime} \in\left(0, s_{i}\right)$ and when $B^{\prime}$ is the ball of radius $s^{\prime}$ centered at $\tau$, then the integral of $\omega$ over $C_{i} \cap B^{\prime}$ is assuredly greater than $2(p+\kappa) s^{\prime 3} / 3$, where $\kappa>0$ is a constant which is independent of the index $i$. Given this last fact, reintroduce the ball $B \subset X$ of radius $s$ centered at $\tau$. The third point of Proposition 2.1 in [T2] implies that the integral of $\omega$ over $C_{i} \cap B$ is greater than $2(p+\kappa) s^{3} / 3$.

This last conclusion is incompatible with the bound by $2(p+\delta) s^{3} / 3$ for the same integral. Thus, $C_{i}$ is in $\mathcal{M}_{b}$ when the index $i$ is large.

e) Proof of the first two assertions of Proposition 2.9 and bounds for $\left\{C_{i}\right\}$. The assertions from Proposition 2.9 are proved in this subsection and in the following one. The first two assertions of Proposition 2.9 are more or less direct consequences of Lemma 3.5 which establish some useful apriori bounds for the sequence $\left\{C_{i}\right\}$. Lemma 3.5 is the focal point in this subsection

To begin the discussion, assume as before only that $C \in \mathcal{M}_{b}$. Fix attention on a component $Z^{\prime}$ of $Z$ and then fix $\varepsilon>0$ as in the previous section. So as to simplify notation, $C$ will also denote the subset of points in the chosen pesudoholomorphic subvariety with $f \leq s^{2}$. (Whether $C$ refer to all or just part of the chosen subvariety should be clear from the context.) 
In any event, as now $f \leq s^{2}$ on $C$, the latter is a smooth submanifold with boundary, and

$$
\begin{aligned}
& \text { - } \int_{C \cap\left\{f \leq s^{2}\right\}} d t \wedge d f=2 \pi p s^{2}, \\
& \text { - } \int_{C \cap\left\{f \leq s^{2}\right\}} d \varphi \wedge d h=4 s^{3} \int \sum_{\alpha}\left|\varphi^{\prime}{ }_{0 \alpha}\right|^{2} d t+\mathcal{O}\left(s^{3}\right) .
\end{aligned}
$$

Meanwhile, in the second line of (3.3), the summation in the integral on the right side is over the $p$ (locally defined) components of $C$. Note that the first line of (3.3) implies that $2 \pi p$ gives the integral of $d t$ over the $f=$ constant curves in $C$. The equalities in (3.3) imply that the area of the portion of $C$ where $r \leq s$ is bounded by $\zeta s$.

Now, consider the restriction to the neighborhood of $Z^{\prime}$ of each $C_{i}$ from the given sequence from $\mathcal{M}$ which converges to $C$. As with $C$, there will be no notational distinction between the whole of $C_{i}$ and the portion near $Z^{\prime}$.

Lemma 3.5, below, summarizes some of the initial observations about each $C_{i}$.

The statement of the Lemma 3.5 introduces, for each index $i$, what will be called the formal metric closure of $C_{i}$. The latter is the compact space, $\underline{C}_{i}$, which is obtained by completing $C_{i}$ using the induced metric from $X$. In this regard, note that (1.4) implies that each end of $C_{i}$ near $Z^{\prime}$ is a smoothly embedded, open annulus in $X-Z$. This implies that the corresponding part of $\underline{C}_{i}$ is a smooth manifold with boundary, and that the tautological embedding of $C_{i}$ into $X-Z$ extends as a smooth map from a neighborhood of the boundary of $\underline{C}_{i}$ into $X$ which sends the boundary to $Z^{\prime}$ as a covering map. There is an equivalent picture of $\underline{C}_{i}$ in terms of the smooth curve model $C_{0 i}$ of Definition 1.1. From this point of view, (1.4) implies that $C_{0 i}$ is the interior of a smooth, compact manifold with boundary, $\underline{C}_{0 i}$. Moreover, the almost everywhere 1-1 pseudoholomorphic map $\sigma_{i}: C_{0 \alpha} \rightarrow X-Z$ whose image is $C_{i}$ extends over $\underline{C}_{0 i}$ to map the latter's boundary to $Z$ as a covering map onto its image. With $\underline{C}_{0 i}$ understood, then a neighborhood of the boundary of $\underline{C}_{0 i}$ is naturally identified with a neighborhood of $\underline{C}_{i}-C_{i}$ in $\underline{C}_{i}$.

LEMMA 3.5. There are constants $\varepsilon>0$ and $\zeta \geq 1$ which have the following significance:

- If $i \geq \zeta$, then $\sigma_{i}$ maps the boundary of $\underline{C}_{i}$ to $Z^{\prime}$ as a $p$ to 1 map.

- If $i \geq \zeta$, then $|z| \leq \rho / 100$ where $r \leq \varepsilon$ on $C_{i}$.

- If $i \geq \zeta$ and if $s \leq \varepsilon$, then the area of $C_{i} \cap\{r \leq s\}$ is bounded by $\zeta s$.

- If $i \geq \zeta$ and if $s \leq \varepsilon$, then the integral over $C_{i} \cap\{r \leq s\}$ of $d \varphi \wedge d h$ is bounded by $\zeta \mathrm{s}^{3}$.

This lemma is proved below.

In the mean time, note that the first point of this lemma gives the first assertion of Proposition 2.9. Also, the second assertion of Proposition 2.9 follows from the second point of Lemma 3.5 with the help of Lemma 3.4. Indeed, it follows from (1.4) that the degree associated to $C_{i}$ by $Z^{\prime}$ is $(2 \pi)^{-1}$ times the integral of the form $d \varphi$ over a level set of $r$ for a sufficiently small, but positive value of the function $r$ on $C_{i}$. (Equation (1.4) insures that sufficiently small values of $r$ on $C_{i}$ are regular values.) Meanwhile, Lemma 3.4 implies that the $r=\varepsilon / 2$ level set is a smooth curve in $C_{i}$ when $i$ is large, and that the integral of $d \varphi$ over this level set is the same as that for the corresponding level set of $C$. Finally, the second point of Lemma 3.5 and Stoke's theorem imply that these two integrals produce $2 \pi$ times the degree associated by $Z$ to $C_{i}$ and $C$, respectively.

Proof of Lemma 3.5. The argument for the first point begins with the observation 
that when $i$ is sufficiently large, then the $r=\varepsilon / 2$ part of $C_{i}$ must also lie where $|z|<\rho / 200$. Indeed, this follows from the pointwise nature of the convergence of $\left\{C_{i}\right\}$ to $C$. In particular, this implies the following: If $i$ sufficiently large, then for all $s<\varepsilon^{2} / 100$, the locus where $f=s$ on $C_{i}$ is compact.

Here is the first implication of the preceding observation: One can fix $s_{0} \in$ $\left(\varepsilon^{2} / 300, \varepsilon^{2} / 200\right)$ to be simultaneously a regular value of the restriction of $f$ to each $C_{i}$. Then, for large $i$, the $C_{i}$ and $s=s_{0}$ version of the first integral in (3.3) must be very close to $2 \pi p s_{0}$. (This follows from Lemma 3.4 using Stoke's theorem.) However, since $d t$ is a closed from whose periods are integer multiples of $2 \pi$, it follows that for sufficiently large $i$, the $C_{i}$ and $s=s_{0}$ version of the top line in (3.3) is precisely $2 \pi p s_{0}$. This implies that the integral of $d t$ over the curve $f=s_{0}$ on $C_{i}$ is also equal to $2 \pi p$ if $i$ is large. But then, if $s<s_{0}$ is a regular value of $f$ on $C_{i}$, the curves $f=s$ and $f=S_{0}$ on $C_{i}$ are homologous on $C_{i}$ as all level sets of $f$ with small values of $f$ were observed to be compact. Thus, the integral of $d t$ over any $f=s$ curve on $C_{i}$ equals $2 \pi p$. In particular, this is true for very small values of $s$, which indicates that $\sigma_{i}$ maps $\sigma_{i}^{-1}\left(Z^{\prime}\right)$ (a subset of $\partial \underline{C}_{0 i}$ ) as a $p$ to 1 map onto $Z$.

To begin the proof of the second point in Lemma 3.5, note that the observation that $|z|<\rho / 200$ where $r=\varepsilon / 2$ on $C_{i}$ also implies that the function $f$ is positive where $r \leq \varepsilon / 2$ on $C_{i}$. Indeed, if $f$ is ever zero on $C_{i}$, then $C_{i}$ has finite, positive intersection number $m$ with some $f=0$ and $t=$ constant pseudoholomorphic subvariety where $r<\varepsilon / 2$. (Note that $C_{i}$ has no such intersections where $r=\varepsilon / 2$ and where $r$ is very small since it eventually approaches $Z$ where $|z|=\mathcal{O}\left(\rho^{2}\right)$.) This implies that for all sufficiently small but positive $\delta$, there are $m$ intersections (counting multiplicity) between $C_{i}$ and the $f=-\delta, t=$ constant pseudoholomorphic subvarieties which occur where $r<\varepsilon / 2$. Since all of these intersections have positive intersection number, these intersections must persist as $\delta$ is increased. Moreover, as $\delta$ is varied, these intersection points must move continuously as a $\delta$-dependent map into the $m$-fold symmetric product of $X$. Even so, none of these intersection points can cross the $r=\varepsilon / 2$ divide (because $f$ is seen to be positive there) and this causes a problem since $r \geq \varepsilon / 2$ on the $f=-\left(\varepsilon^{2} / 4\right), t=$ constant subvarieties.

To continue with the proof of the second point of Lemma 3.5, observe that if $f>0$ on $C_{i}$ where $r \leq \varepsilon / 2$, then one can introduce the function $u \equiv(2 f)^{1 / 2}$ on the $r \leq \varepsilon / 2$ portion of $C_{i}$. Together the pair $(t, u)$ restrict as local coordinates in a neighborhood of almost every point on the $r \leq \varepsilon / 2$ portion of $C_{i}$. Note that the critical points of the restriction of $u$ agree with those of the restriction of $t$. (This follows from the fact that $C_{i}$ is pseudo-holomorphic.)

Given the preceding, introduce the function $v \equiv h / u^{3}=z / u+2 z^{3} / u^{3}$. This function is bounded on $C_{i}$ where $r \leq \varepsilon / 2$ and tends to zero as $Z$ is approached. (The function $v$ is very small at all points sufficiently near $Z$ on $C_{i}$ since (2.1) also described each $C_{i}$ on some neighborhood of $Z$.) The claim here is that $v$ has no positive maximum nor negative minimum on $C_{i}$ where $r \leq \varepsilon / 2$. Since $v$ tends to zero near $Z$ on $C_{i}$ and, where $i$ is large, $v$ is less that $1 / 150$ on the $r=\varepsilon / 2$ divide, it follows that $v<150$ on all of the $r \leq \varepsilon / 2$ portion of $C_{i}$ when $i$ is large. This implies the second assertion of Lemma 3.5.

To see that $v$ has no positive maximum (or negative minimum), first assume that such a point occurs in $C_{i}$ where $(t, u)$ are good coordinates. In this regards, note that the restriction to $C_{i}$ of the pair $(\varphi, v)$ obey the following equation:

- $v_{u}+3 v / u-\left(1+\kappa_{1}\right) \varphi_{i}=0$.

- $v_{t}+\left(1+\kappa_{2}\right) \varphi_{u}=0$. 
Here, the subscripts denote partial differentiation by the indicated variable. Also, $\kappa_{1}=\rho^{2} / u^{2}-1$ and $\kappa_{2}=g^{2} \rho^{2} / u^{4}-1$. In particular, both $\kappa_{1,2}$ are functions of $z^{2} / u^{2}$ and thus both can be viewed as smooth functions of the function $v^{2}$ which are analytic when $v^{2}<1 / 100$. (In this regard, remember that $v$ is defined in terms of the function $\lambda \equiv z / u$ by the formula $v=\lambda+2 \lambda^{3}$. Since the derivative $v_{\lambda}=1+6 \lambda^{2}>0$, this relationship can be inverted to give $\lambda$ as a smooth function of $v$.)

In any event, if follows by differentiating (3.4) that

$$
\left(1+\kappa_{1}\right)^{-1}\left(v_{u u}-3 v / u^{2}\right)+\left(1+\kappa_{2}\right)^{-1} v_{t t}=0
$$

at a critical point of $v$ where $(t, u)$ are good coordinates. This last equation implies that $v$ has no positive maxima nor negative minima where $(t, u)$ are good coordinates.

Now consider points where $(t, u)$ are not good coordiantes. If such a point is a manifold point of $C_{i}$, then $d v \neq 0$ there, since the 2 -form $\omega$ at such a point is given by $u^{3} d \varphi \wedge d v$. Meanwhile, the possibility of a local maximum at a singular point of $C_{i}$ can be ruled out using the local picture of such points which is proved in Part a of the Appendix in [T2]. Indeed, the argument in this case follows directly from the analog of (3.5) which is obtained by differentiating Equation (A.5) in [T2] with respect to the complex variable $v$ as defined using (A.6) in [T2] and then examining the result at $\mathrm{v}=0$.

The third pint in Lemma 3.5 follows directly from Proposition 2.1 in [T2] in as much as the integral of $\omega$ over $C_{i}$ is uniformly bounded.

The fourth point in Lemma 3.5 is obtain from (5.4) in [T2] with the observation that the constant $\zeta$ which appears there for each $C_{i}$ can be bounded by an index $i$ independent constant which is determined by points in $C$ which are uniformly far from $Z$. Indeed, this assertion follows from (5.6) in [T2] with the remark that the bounds given in [T2] for each of the terms in this equation are determined by the behavior of the restriction of the 1-form $d \varphi$ to points of $C_{i}$ which are uniformly far from $Z$ and near manifold points of $C$. In particular, Lemma 3.4 insures that the bounds which are so obtained for $C_{i}$ are, for large $i$, within a fixed factor, say 2 , of those which are obtained for $C$ by the same arguments.

f) Proof of Proposition 2.9, completion. This subsection completes the proof of Proposition 2.9; the as yet unproved assertion is a corollary to Lemma 3.6 below. The latter provides some additional uniform estimates for the sequence $\left\{C_{i}\right\}$.

Before stating Lemma 3.6, it proves useful to digress to set the stage. To begin the digression, note that as remarked in the previous step, the function $u=(2 f)^{1 / 2}$ is well defined on $C$ where $r \leq \varepsilon$; thus where $r \leq \varepsilon$ and $i$ is large, there is a map from $C_{i}$ to $S^{1} \times(0, \varepsilon]$ given by the values of the functions $t$ and $u$. The latter map extends to one from $\underline{C}_{i}$ to $S^{1} \times[0, \varepsilon]$. Here, $u=0$ corresponds to the boundary components of $\underline{C}_{i}$ near $Z^{\prime}$, and then $t$ on the $u=0$ locus maps the latter as a $p$ to 1 cover of $S^{1}$. (As in the previous section, there will be no notational distinction between the portion of $C_{i}$ near $Z^{\prime}$ where $u \leq \varepsilon$ and the whole of $C_{i}$; the distinction should be clear from the context.)

As the inverse image of all but finitely many values $(t, u)$ on $C_{i}$ consists of $p$ points, the tautological map from where $r \leq \varepsilon$ in $\underline{C}_{i}$ into $X$ is determined by an unordered set of $p$ (locally defined) pairs of functions $\left\{\varphi_{i, \alpha}, v_{i, \alpha}\right\}_{1 \leq \alpha \leq p}$. Here, the ordering and thus the labeling of the elements of $\left\{\left(\varphi_{i,}, v_{i, .}\right)\right\}$ may not be well defined. Indeed, a consistent labeling is assured only in some small neighborhood of any point where these pairs are distinct. This set can also be viewed as the push-forward via 
the functions $(t, u)$ to $S^{1} \times[0, \varepsilon]$ of the functions $\left(\varphi, v \equiv h / u^{3}\right)$ and the latter view will now be taken.

At times, it will prove convenient to consider $\left\{\varphi_{j},\right\}$ as a map from $S^{2} \times[0, \varepsilon]$ into the $p^{\prime}$ th symmetric product of $S^{1}$. For this purpose, introduce the polynomial $\mathcal{P}_{j}(\lambda)=$ $\Pi$. $\left.\lambda-\exp \left(i, \varphi_{j, .}\right)\right)$ in the variable $\lambda$. Expand the latter as $\mathcal{P}(\lambda)=\lambda^{p}+a_{1} \lambda^{p-1}+\cdots+a_{p}$, and the coefficients provide a well defined map $\Phi_{j}: S^{1} \times[0, \varepsilon] \rightarrow \mathbb{C}^{p}$ which determines the set $\left\{\varphi_{j,},\right\}$ as a map into $\operatorname{Sym}^{p}\left(S^{1}\right)$. (These coefficients of $\mathcal{P}$ determine the set $\left\{\varphi_{j,},\right\}$ up to ordering and up to adding a multiple of $2 \pi$ to any element.)

With the digression now at its end, consider:

Lemma 3.6. Let $\varepsilon>0$ be as in the previous lemma. There is a constant $\zeta \geq 1$ which has the following significance:

- If $i \geq \zeta$, then $|v| \leq \zeta u$ where $r \leq \varepsilon$ on $C_{i}$.

- If $i \geq \zeta$ and if $s \in(0, \varepsilon)$ and if $D$ is the $u \geq 0$ part of a disk of radius $s$ in $S^{1} \times[-\varepsilon, \varepsilon]$, then

$$
\int_{D} \Sigma .\left(\left|d \varphi_{j, \cdot}\right|^{2}+\left|d v_{j,}\right|^{2}\right) \leq \zeta s^{1 / \zeta}
$$

- The sequence of maps $\left\{\Phi_{i}: S^{1} \times[0, \varepsilon] \rightarrow \mathbb{C}^{p}\right\}$ is uniformly equicontinuous.

Lemma 3.6 implies directly the final assertion of Proposition 2.9. Indeed, it follows from the third point of Lemma 3.6 that the sequence $\left\{\Phi_{i}\right\}$ converges uniformly on $S^{1} \times$ $[0, \varepsilon]$ to the corresponding map $\Phi$ as analogously defined by the pseudoholomorphic submanifold $C$. Here, Lemma 3.4 insures that $\left\{\Phi_{j}\right\}$ converges to $\Phi$ on compact subsets of $S^{1} \times(0, \varepsilon]$ and then the fact that the sequence is uniformly equicontinuous on the compact domain $S^{1} \times[0, \varepsilon]$ implies that it must also converge to $\Phi$ on a neighborhood of $Z=S^{1} \times\{0\}$.

Proof of Lemma 3.6. The lemma's first point follows directly from the second point of Lemma 3.5 using the argument in [T2] which proves Eq. (8.4) in [T2]. Lemma 3.6's second point then follows from the first point using the argument in [T2] which proves Lemma 8.2 in [T2]. The final point then follows from the second point here using the argument in [T2] which proves Lemma 8.3 in [T2].

g) Properties of $\left\{\varphi_{i,}\right\}$ as defined by elements from $\left\{C_{i}\right\}$. This subsection serves as a digression of sorts to further describe the behavior of the sequence $\left\{\left(\varphi_{i, .}, v_{i,}\right)\right\}$ as defined using the sequence $\left\{C_{i}\right\}$. Here, the behavior of this sequence will be considered in a neighborhood of a point on $Z$ with the following property:

The point has a ball neighborhood which, when $i$ is large, intersects each $C_{i}$ as a union of $p$ irreducible components; here, $p$ is the number of components of the intersection of the given ball with $C$.

(Remember that an irreducible component of the intersection of $C$ with an open set is the closure in this open set of a component of the set of smooth points of $C$.)

Note that every point in $Z$ satisfies (3.7) in the case where $p=1$. Meanwhile in the case where $p>1$, the final assertion of Proposition 2.9 insures that all but finitely many points in $Z$ obey (3.7). Indeed, when $p>1$, (3.7) can fail only at points in $Z$ where the polynomial $\mathcal{P}$ which defines the map $\Phi$ from the given pseudoholomorphic subvariety $C$ has coincident roots.

In any event, the point of (3.7) is as follows: Near a point where (3.7) holds, there is, for all $i$ sufficiently large, an unambiguous labeling of the set of pairs $\left\{\left(\varphi_{i, \cdot}, v_{i,}\right)\right\}$ 
which turns the latter into an ordered sequence of pairs of functions on the intersection of the $u \geq 0$ portion of the $t$ - $u$ plane with a disk whose center has the $t$-coordinate of the given point on $Z$ and $u=0$. Moreover:

LEMMA 3.7. Fix a point in $Z$ where (3.7) holds. For all $i$ sufficiently large, a labeling can be found for $\left\{\left(\varphi_{i, .}, v_{i,}\right)\right\}$ on a neighborhood of the point in question so that the each of the resulting $p$ sequences of pairs of functions converges as a sequence of pairs of real analytic functions on the intersection of the $u \geq 0$ half of the $t$ - $u$ plane with a disk centered at the point with the $t$-coordinate of the given point in $Z$ and $u=0$.

Proof of Lemma 3.7. As remarked above, when (3.7) holds, the set $\left\{\left(\varphi_{i, .}, v_{i, .}\right)\right\}$ can be unambiguously labeled for large $i$ on a fixed neighborhood of the origin in the $t-u$ plane. Do so, and focus attention on one such labeled pair, $\left(\varphi_{i}, v_{i}\right)$. For each $i$, the corresponding pair consists of real analytic functions on the intersection of the $u \geq 0$ part of the $t-u$ plane with a disk $D$ whose center has the $t$-coordinate of the given point in $Z$ and $u=0$. This assertion is proved in Section 8b of [T2]. The proof, after a rewriting of (3.4), is no more than an appeal to the standard theorems about regularity of solutions to elliptic equations. In particular, as these theorems are proved by establishing apriori bounds for derivatives, they automatically imply the asserted convergence of the sequence $\left\{\left(\varphi_{i}, v_{i}\right)\right\}$ as a sequence of pairs of real analytic functions.

h) The proof of Proposition 2.10. The proof of Proposition 2.10 depends on a key lemma (Lemma 3.8) which concerns the topology near $Z$ of subvarieties $C^{\prime} \in \mathcal{M}_{b}$ which are close to the given variety $C$. In this regard, note that the topology near a component $Z^{\prime}$ of $Z$ of a subvariety in a $p=1$ component of $\mathcal{M}_{b}$ is necessarily constant, as each such subvariety is properly diffeomorphic near $Z^{\prime}$ to an annulus. Indeed, it follows from Lemma 3.5 and the subsequent discussions that when $C \in \mathcal{M}_{b}$ has $p=1$, then there exists $\varepsilon>0$ and a neighborhood of $C$ in $\mathcal{M}_{b}$ with the following significance: If $C^{\prime}$ comes from the given neighborhood, then the conclusions of Lemma 3.5 hold for $C^{\prime}$. In particular, this implies that the $r \leq \varepsilon / 2$ portion of $C^{\prime}$ is a graph over the $(t, u)$ plane; that is, the functions $(t, u)$ restrict to the $r<\varepsilon / 2$ region of $C^{\prime}$ as good coordinates.

On the other hand, if $C$ has $p>1$, then there may or may not be a fixed $\varepsilon>0$ and neighborhood of $C$ in $\mathcal{M}_{b}$ such that $(t, u)$ are good local coordinates on the $r<\varepsilon / 2$ portion of any $C^{\prime}$ from the given neighborhood. Examples are given in the next subsection where such is definitely not the case. In any event, were there such an $\varepsilon$, then (3.7) would hold at all points of $Z$ for any sequence $\left\{C_{i}\right\} \subset \mathcal{M}_{b}$ which converges to $C$.

However, when $p>1$, it turns out that there exists $\varepsilon>0$ and an apriori bound on the topological complexity of the $r<\varepsilon / 2$ portion of any $C^{\prime} \subset \mathcal{M}_{b}$ which is near to $C$. This is made precise in Lemma 3.8, below. Prior to reading this lemma, the reader should note the following consequence of (1.4): To each $C^{\prime} \in \mathcal{M}_{b}$ there is a corresponding smooth, complex curve $C^{\prime}{ }_{0}$ with a proper, almost everywhere 1-1, pseudoholomorphic map $\sigma^{\prime}: C^{\prime} \rightarrow X-Z$ whose image is $C^{\prime}$.

Lemma 3.8. Fix $C \in \mathcal{M}_{b}$. Then there exists $\varepsilon>0$, an integer $N$ and a neighborhood of $C$ in $\mathcal{M}_{b}$ with the following significance: First, if $C \in \mathcal{M}_{r b}$, then $N=0$. In any event, let $C^{\prime}$ lie in the given neighborhood; let $C^{\prime}{ }_{0}$ denote the smooth model for $C^{\prime}$, and let $\sigma^{\prime}: C^{\prime}{ }_{0} \rightarrow X$ denote the almost everywhere 1-1, pseudoholomorphic map whose image is $C^{\prime}$. Then, both $t$ and $u=(2 f)^{1 / 2}$ are well defined on the $r<\varepsilon / 2$ 
portion of $C^{\prime}$. Moreover, the pull back via $\sigma^{\prime}$ of either $t$ or $u$ to $C^{\prime}$, has no more than $N$ critical points.

This lemma is proved below. (A precise bound on $N$ comes from Lemma 4.2 and the discussion in Steps 8 and 9 of Section $4 \mathrm{~b}$ to come.)

Consider first using Lemma 3.8 for the

Proof of Proposition 2.10. As $N=0$ for $C \in \mathcal{M}_{r b}$, it follows from Lemma 3.4 and Lemma 3.8 that $C$ has a neighborhood $\mathcal{U} \subset \mathcal{M}_{b}$ and each point in $Z$ has a ball neighborhood such that the restriction of each $C^{\prime} \subset \mathcal{U}$ to the given ball is a disjoint union of $p$ components. That is, every sequence $\left\{C_{i}\right\} \subset \mathcal{M}_{b}$ which converges to $C$ obeys (3.7) at each point in $Z$. With this understood, the conclusions of Proposition 2.10 follow immediately from Lemma 3.7.

This subsection ends with the

Proof of Lemma 3.8. First of all, Lemma 3.5 insures that there exists $\varepsilon>0$ a neighborhood in $\mathcal{M}_{b}$ of $C$ such that $t$ and $u$ are well defined on the $r<\varepsilon / 2$ portion of $C^{\prime}$ from the given neighborhood.

With this understood, consider first Lemma 3.8's assertion that there is a bound to the topological complexity of $C_{i}$ near $Z$. For this purpose, suppose the contrary, that no such bound exists. Then, there exists a sequence $\left\{C_{i}\right\} \subset \mathcal{M}_{b}$ which converged to $C$ but which did not admit a uniform bound on the number of critical points of the pull-back of $t$ and $u$ to the corresponding $C^{\prime}{ }_{i}$. With this sequence $\left\{C_{i}\right\}$ understood, a three step argument provides the necessary contradiction.

Step 1: It follows from Lemma 3.7 that there is a neighborhood of each point where (3.7) holds which lacks critical points of $(t, u)$ on any $C_{i}$ with $i$ sufficiently large. With this understood, one need only focus attention on $C_{i}$ near points in $Z$ where the polynomial $\mathcal{P}$ as defined using $C$ has coincident roots. Fix one such point and agree on coordinates so that its $t$ value is zero. Now, focus on the restriction of $C$ and of each $C_{i}$ to a neighborhood of this points.

Step 2: As in the Appendix to [T2], it proves useful to change variables from $v$ to a function $b=b(v)$ which is defined in (A.1) of [T2]. Here, $b$ is an analytic function of $v$ when the latter is small, and in particular, $b(v)=v+\mathcal{O}\left(v^{3}\right)$. With $b$ understood, introduce the complex valued $\eta \equiv \varphi+i \cdot b$. By virtue of (3.4), this function obeys the nonlinear version of the $\partial$-bar equation in (A.2) of [T2].

Next, introduce the completion, $\underline{C}$, of $C$ with respect to the induced metric from $X$. As attention is restricted below to where $u \leq \varepsilon$ on $C$, the space $\underline{C}$ is a manifold with two boundary components, which properly fibers over $S^{1} \times[0, \varepsilon]$ with fiber degree $p$. (In this case, $\underline{C}$ is the same as the closure of the model $C_{0}$.)

Let $\pi$ denote the projection from $\underline{C}$ to $S^{1} \times[0, \varepsilon]$ and consider $\pi^{*} \underline{C}$ as a fiber bundle over $\underline{C}$. The latter space consists of triples $\left(w=t+i \cdot u, \eta_{-}, \eta_{+}\right)$, where each $\left(w, \eta_{ \pm}\right) \in \underline{C}$. Note that $\underline{C}$ embeds canonically in $\pi^{*} \underline{C}$ as the set of triples of the form $(w, \eta, \eta)$, and often $\underline{C}$ will be identified with is image in $\pi^{*} \underline{C}$ via this embedding. Note that the closure of $\pi^{*} \underline{C}-\underline{C}$ in $\pi^{*} \underline{C}$ adds only certain points of $\underline{C}$ which lie over $Z=S^{1} \times\{0\}$. Indeed, this closure adds only points over $t \in S^{1}$ where the polynomial $\mathcal{P}_{0}$ has coincident roots, and the added points correspond precisely to these coinciding roots.

Now introduce the function $\Delta$ on $\pi * \underline{C}-\underline{C}$ whose value at $\left(w, \eta_{-}, \eta_{+}\right)$is $\eta_{-}-\eta_{+}$. Use $\underline{\varphi}$ and $\underline{b}$ to denote the respective real and imaginary parts of $\triangle$. Note that the 
function $\triangle$ is non-zero on $\pi^{*} \underline{C}-\underline{C}$. However, the function extends to the closure of $\pi^{*} \underline{C}-\underline{C}$ in $\underline{C}$, and its zero set on this closure is exactly the set of points in $\underline{C}$ which are added in the closure operation.

As in Step 3 of Part b of the Appendix to [T2], this $\triangle$ will be used to define an embedded oriented graph $\Gamma$ in the closure of $\pi^{*} \underline{C}-\underline{C}$. Here, $\Gamma$ will be defined only over some small neighborhood of a point $o \in S^{1} \times[0, \varepsilon]$ where two or more roots of the polynomial $\mathcal{P}_{0}$ coincide. In particular, take this to be a closed half disk $D$ of radius less than $\varepsilon$ whose straight edge lies on $S^{1} \times\{0\}$ and center lies at the point in question, $o$. Moreover, take the disk radius small so that the point $o$ is the only point on the straight edge of $D$ where $\mathcal{P}_{0}$ has coincident roots.

By the way, note that over $D-o$ there are $p \cdot(p-1)$ components of $\pi^{*} \underline{C}-\underline{C}$, where each component is diffeomorphic via the projection to $D-o$. These components correspond to an ordered pair of distinct components of $\left.\underline{C}\right|_{D-o}$.

Here is how to define $\Gamma$ (see Lemma A.4 in [T2]): As a set, $\Gamma$ consists of the zero of the function $\underline{b}$. The vertices are the $\underline{b}=0$ critical points of $\underline{b}$ and also the points of $\left.\underline{C}\right|_{Z}$ which are added when closing $\pi^{*} \underline{C}-\underline{C}$. And, by taking the radius of $D$ to be very small, one can insure that only the latter sort of vertices appear. In fact, except for some minor notional issues, no generality is lost by assuming that $\Gamma$ has just a single vertex, and this assumption will now be made. (The graph $\Gamma$ would have two or more vertices in this situation when $\mathcal{P}_{0}$ has two or more distinct multiple roots at the given point in $Z$. Since different roots of $\mathcal{P}_{0}$ correspond, at least locally, to different components of $C$, the essentially local analysis that follows can be applied separately to each of the corresponding parts of $\pi^{*} \underline{C}$.)

As in Part b of the Appendix to [T2], orient each edge of $\Gamma$ by requiring the positivety of the restriction of the 1 -form $d \varphi$.

The behavior of $\Gamma$ near the vertex in question is described in Lemma A.5 of [T2] and in the subsequent remark: Let $D^{\prime} \subset \pi^{*} \underline{C}-\underline{C}$ denote a component whose closure in $\pi^{*} \underline{C}$ contains the vertex. (Remember that $D^{\prime}$ is diffomorphic to $D-o$ ). Then, there are some $m+1$ edges of $\Gamma$ in $D^{\prime}$ which emanate from the vertex. Here $m$ is the order of vanishing of $\varphi$ as one moves through the vertex along the inverse image of the $u=0$ line in $D^{\prime}$. (Thus, $m-1$ is the order of vanishing of $d \varphi$ as one moves through the vertex along the same path). Two of these edges lie over the $u=0$ line, and the remainder are slight deformations of straight arcs which run from the center of $D^{\prime}$ through the interior of $D^{\prime}$ to the circular part of the boundary of $D^{\prime}$.

As noted, all of the edges are naturally oriented by $d \underline{\varphi}$ and as one precedes around the boundary of $D^{\prime}$, the orientations reverse as successive edges are encountered. (They alternate pointing in and out of $D^{\prime}$.)

Step 3: For large $i$, make the analogous construction of $\underline{C}_{i}$ and $\pi^{*} \underline{C}_{i}$; then use the construction in Part b of the Appendix to [T2] to build the analogous oriented graph $\Gamma_{i}$. (The closure of $\pi^{*} \underline{C}_{i}-\underline{C}_{i}$ in $\underline{C}_{i}$ contains $\Gamma_{i}$.) Fix a disk neighborhood $B \subset D$ of the center point $o$ which is concentric to $D$, but with much smaller radius. Then, when $i$ is large, $\left.\pi^{*} \underline{C}_{i}\right|_{D-B}$ is diffeomorphic to $\left.\pi^{*} \underline{C}\right|_{D-B}$ and this diffeomorphism carries $\Gamma_{i}$ to $\Gamma$ and preserves the orientations. Thus, for large $i$, any extra vertices of $\Gamma_{i}$ must lie over $B$.

With the preceding understood, the argument in Step 10 of Part b of the Appendix to [T2] provides an index $i$ independent bound to the number of extra vertices of $\Gamma_{i}$. In this regard, note that the arguments in Step 10 of Part b of appendix in [T2] require the 1-form $d \varphi$ to restrict to $\left.C_{i}\right|_{D}$ as an exact form. This happens automatically if the variation of $\varphi$ on $\left.C_{i}\right|_{D}$ is smaller than $2 \pi$, and the latter is insured for large $i$ through 
Proposition 2.6 provided that $D$ is chosen so that $\varphi$ on $\left.C\right|_{D}$ has small variation.

The bound from Step 10 of Part b of the Appendix in [T2] implies that there is an index $i$-independent bound to the number of points in the set $\Lambda_{i} \subset C_{i}$ which are either singular points of $C_{i}$ or else critical points of $u$ (and $t$ ). This is to say that there is an $i$ independent bound to the number of critical points on the model curve $C_{i 0}$ of the pulled back functions. This conclusion contradicts the initial assumption about the sequence $\left\{C_{i}\right\}$.

Now consider the assertion in Lemma 3.8 that $N=0$ when $C \in \mathcal{M}_{r b}$. Indeed, this follows immediately from the discussion in Step 10 of Part b of the Appendix in [T2] since in this case, the graphs $\Gamma$ which are constructed project to the $t-u$ plane with image the $u=0$ line.

i) Topology changes near $Z$. The purpose of this subsection is to provide some (local) examples which exhibit a pseudoholomorphic submanifold $C$ with nearby pseudoholomorphic submanifolds that have different topology near $Z$. These examples are 'local' in the sense that they are defined only in a neighborhood of $Z=S^{1} \times\{0\}$ in the coordinate system on $S^{1} \times B^{3}$ given in (1.1). Presumably, these local examples also occur in the context where $X$ is compact. In the examples below, $p=2$ and the pseudoholomorphic subvarieties are defined by giving a set of pairs $\left\{\left(\varphi_{\alpha}, v_{\alpha}\right)\right\}_{1 \leq \alpha \leq 2}$ of the variables $(t, u)$ which are defined near $u=0$ and obey (3.4).

In these examples, take $\left(\varphi_{1}, v_{1}\right)=(0,0)$. Fix a positive integer $q$. Since $t \rightarrow$ $\sin ^{q} t$ is a real analytic function of $t$, there exists $\varepsilon>0$ and a pair of functions $\left(\varphi_{2}(t, u), v_{2}(t, u)\right)$ which are defined where $0 \leq u<\varepsilon$, which obey $\varphi_{2}(t, 0)=\sin ^{q} t$ and $v_{2}=(t, 0)=0$, and which solve (3.4). The subvariety $C$ defined by the pair $\left\{\left(\varphi_{\alpha}, v_{\alpha}\right)\right\}_{1 \leq \alpha}$ is, near $u=0$, a submanifold that consists of a union of two annuli. Indeed, to prove that $C$ is a submanifold where $u>0$ but small, it is enough to prove that the two sheets of $C$ do not intersection. In this regard, the only worry is near $t=0$ or $t=\pi$, and $u=0$, and a short digression follows to examine the behavior of $C$ near $t=0$ and $u=0$.

To start the digression, change coordinates to polar coordinates $(s, \theta)$ in the $t-u$ plane (so that $t=s \cos \theta$ and $u=s \sin \theta$ ). In these coordinates, power series expansion for $\varphi_{2}$ and $v_{2}$ read

- $\varphi_{2}(t, u)=s^{q} a_{q}(\cos \theta)+\mathcal{O}\left(s^{q+2}\right)$

- $v_{2}(t, u)=(q+3)^{-1} s^{q} \sin \theta a^{\prime}{ }_{q}(\cos \theta)+\mathcal{O}\left(s^{q+2}\right)$

Here, $a_{q}(\cdot)$ is a polynomial of degree $q$ with the same parity as $q$, while $a^{\prime}{ }_{q}$ denotes the derivative of $a_{q}$. To be more precise, $a=a_{q}$, as a function of $w \in[-1,1]$, is the unique solution to the differential equation

$$
\left(1-w^{2}\right)^{-1}\left(\left(1-w^{2}\right)^{2} a^{\prime}\right)^{\prime}+q(q+3) a=0
$$

which obeys $a_{q}(1)=1$. For example, the relevant $q=0,1,2$ and 3 solutions are: $a_{0}(w)=1, a_{1}(w)=w, a_{2}(w)=\left(5 w^{2}-1\right) / 4$, and $a_{3}(w)=\left(7 w^{3}-3 w\right) / 4$.

In any event, as the only solution to (3.9) with both $a$ and $a^{\prime}$ zero at the same point has $a=0$, both $\varphi_{2}$ and $v_{2}$ in (3.8) vanish simultaneously near $s=0$ only at $s=0$.

With the digression now at an end, consider the subvariety $C^{\prime}$ which is obtained by perturbing the $u=0$ boundary values of $\varphi_{2}$ to $\sin ^{q} t+\delta$, where $\delta$ is a small real number. The resulting subvariety $C^{\prime}$ is defined by $\left(\varphi_{1}, v_{1}\right)=(0,0)$ as before, and $\left(\varphi^{\prime}{ }_{2}, v_{2}^{\prime}\right)$.

Note that for small $\delta$, the subvariety is a submanifold where $u$ is small expect possibly near $t=0$ or $t=\pi$ where its two sheets are close to each other. Near $t=0$ 
and $u=0$, the behavior of $C^{\prime}$ can be analyzed by writing the analogous power series expansion to (3.9). Through order $s^{q}$, the resulting expansion finds:

- $\varphi_{2}^{\prime}=s^{q} a_{q}(\cos \theta)+\delta+\mathcal{O}\left(s^{q+2}\right)$,

- $v_{2}^{\prime}=(q+3)^{-1} s^{q} \sin \theta a^{\prime}{ }_{q}(\cos \theta)+\mathcal{O}\left(s^{q+2}\right)$.

Thus, when $\delta$ has small norm, the two sheets of $C^{\prime}$ intersect near $s=0$ at the points whose coordinates $(s, \theta)$ are such that $a_{q}^{\prime}(\cos \theta)=0$ and $s=\left(-a_{q}(\cos \theta)^{-1} \delta\right)^{1 / q}$. Thus, the intersection points of the two sheets of $C^{\prime}$ are determined by the critical points of $a_{q}$ which have critical value whose sign is opposite to that of $\delta$. For example, in the case where $q=1$, there are no critical points at all, where as in the case $q=2$, there is one critical point of $a_{q}$ (where $w=0$ ), and here $a_{2}=-1 / 4$, so the sheets intersect when $\delta>0$, but they do not when $\delta<0$.

In general, the maximum principle with (3.9) implies that the positive critical values correspond to local maxima and the negative ones to local minima of $a_{q}$. Moreover, there are $q-1$ critical points in all; and $q / 2$ of them have $a_{q}<0$ when $q$ is even, while $(q-1) / 2$ have $a_{q}<0$ when $q$ is odd. Thus, even so $C^{\prime}$ is still a union of two annuli near $u=0$, these annuli now intersect some number of times.

In the previous example, $C^{\prime}$, though possibly immersed, is still the union of two embedded annuli as was the unperturbed version with $\delta=0$. Thus, the irreducible components of $C^{\prime}$ do not change their topology as $\delta$ is varied from zero. However, an example where the topology of the irreducible components do change topology when $\delta$ is non-zero can be constructed from $C^{\prime}$. To do so, fix $\delta$ and construct $C^{\prime}$ as above. Focus attention on the intersection points between the two sheets. In any specified neighborhood of these intersection points, the intersections can be resolved to give a connected submanifold $C^{*}$ which agrees with $C^{\prime}$ outside of the given neighborhood. This submanifold is obtained from the two annuli by adding 1-handles between them, where each self intersection point of $C^{\prime}$ accounts for precisely one of the added handles. (Thus, the genus of $C^{*}$ is one less than the number of self intersection point of $C^{\prime}$.)

Specify a neighborhood of the double points of $C^{\prime}$ and a standard construction modifies $C^{\prime}$ to produce a closed, symplectic submanifold which agrees with $C^{\prime}$ outside of the given neighborhood. In addition, if the given neighborhood of the double points is a union of sufficiently small radii ball, then this new symplectic submanifold can be perturbed so that the result is back in $\mathcal{M}_{r}$. This last perturbation will not generally be compactly supported away from $Z$. None-the-less, the resulting submanifold will be closed to $C$ in $\mathcal{M}_{r}$ if $|\delta|$ is small and if the resolution of the double points of $C^{\prime}$ are done in sufficiently small radii balls. These last claim are not meant to be obvious. In any event, they can be proved by employing the Fredholm theory outlined in Proposition 2.6 and Section 5 to define the pseudoholomorphic perturbation of the symplectic resolution of the singular $C^{\prime}$. The details for this argument are omitted as the discussion would be lengthy and mostly tangential to the story line here.

4. Proofs of Proposition 2.5 and 2.7. This section contains the proof of Proposition 2.5 and then that of Proposition 2.7. The proof of Proposition 2.5 has two parts. Part 1 considers the assertion that $\mathcal{M}_{b}=\mathcal{M}_{b, 0}$, while Part 2 considers the closure of $\mathcal{M}_{b, k+1}$ in $\mathcal{M}_{b, k}$.

a) The proof that $\mathcal{M}_{\boldsymbol{b}}=\mathcal{M}_{\boldsymbol{b}, 0}$. The assertion that these two space are equal is equivalent to the assertion that $k(C)$ from (2.2) is non-negative. Meanwhile, the latter assertion ultimately stems from the fact, first observed by Gromov [Gr] and $\mathrm{McDuff}[\mathrm{McD}]$, that the local sign of intersection between pseudoholomorphic subvarieties is positive. In any event, the detailed argument for non-negativity of the 
right side of (2.2) follows.

To begin, perturb the map $\sigma$ away from $\partial C_{0}$ so that the resulting map, $\sigma^{\prime}$, is both symplectic and an immersion on $\operatorname{int}\left(C_{0}\right)$. For such a perturbation, the sum of the signs associated to the self-intersection points of $\sigma^{\prime}\left(C_{0}\right)$ is non-negative and strictly positive if $\sigma$ is not 1-1. (The latter assertion follows from [McD]. The fact that $\sigma^{\prime}$ can be made symplectic follows from the known structure of $\sigma^{\prime}$ near its critical points as presented in [Ye], [PW] or [MS].).

Each of the terms on the right hand side of (2.2) can be computed using the map $\sigma^{\prime}$ instead of $\sigma$ (with the immersed submanifold $C^{\prime}=\sigma^{\prime}$ (int $\left(C_{0}\right)$ ) replacing $\sigma$ ). In particular, use the fact that $C^{\prime}$ is immersed to write $\sigma^{*} T X=T C_{0} \oplus N$. It then follows that $c_{1}\left(\sigma^{*} T X\right)=c_{1}\left(\sigma^{*} T X\right)=\chi\left(C_{0}\right)+d_{c^{\prime}}$, where $d_{c^{\prime}}$ is the degree of the normal bundle $\sigma^{*} N_{c^{\prime}} \rightarrow C_{0}$ as defined using the section on $\partial C_{0}$ that is described in Step 4 of Section $2 \mathrm{~b}$. Thus, the right hand side of $(2.2)$ is equal to $k\left(C^{\prime}\right) \equiv C^{\prime} \bullet C^{\prime}-d_{c^{\prime}}$.

Here is how to compute $k\left(C^{\prime}\right)$ : Remark first that there exists a tubular neighborhood $\mathcal{N} \subset X$ of $Z$ in which $C^{\prime}=C$ and neither has any immersed points. Indeed, the existence of such a neighborhood follows from (1.4). Second, if the section over $\partial C_{0}$ has small enough norm, then the resulting push-off, $C^{\prime \prime}$ of $C^{\prime}$ can be constructed to have no intersection points with $C^{\prime}$ in $\mathcal{N}$ save in a neighborhood, $\mathcal{N}^{\prime} \subset \mathcal{N}$ which is the union of small balls, one for each point $t \in Z$ where a distinct pair $\left(\varphi_{0 \alpha^{\prime}}, \varphi_{0 \alpha^{\prime \prime}}\right)$ from (1.4)'s data set $\left\{\varphi_{0 \alpha}\right\}$ coincide. Moreover, the radii of these balls can be fixed in advance. With the preceding understood, it follows that $k_{c^{\prime}}$ is the sum of two contributions. The first contribution is twice the algebraic sum of the immersion points of $C^{\prime}$. As remarked previously, this sum is always non-negative and positive unless the original map $\sigma$ is everywhere 1 to 1 . The second contribution is the intersection number between $C^{\prime \prime}$ and $C^{\prime}$ in $\mathcal{N}$. The following lemma describes the latter:

LEMMA 4.1. When the balls making up $\mathcal{N}^{\prime}$ have sufficiently small radius, and when $C^{\prime \prime}$ is then defined so its intersections with $C^{\prime}$ in $\mathcal{N}$ take place in $\mathcal{N}^{\prime}$, then the algebraic intersection number between $C^{\prime}$ and $C^{\prime \prime}$ in $\mathcal{N}$ is the same as the geometric intersection number. In fact, this intersection number is a sum of contributions, one from each pair $\left(t,\left\{\varphi_{0 \alpha^{\prime}}, \varphi_{0 \alpha^{\prime \prime}}\right\}\right)$ where $t \in Z$ and where $\left\{\varphi_{0 \alpha^{\prime}}, \varphi_{0 \alpha^{\prime \prime}}\right\}$ is an unordered two component subset of (1.5)'s data set $\left\{\varphi_{0 \alpha}\right\}$ whose values coincide at $t$. The contribution of such a pair to the intersection number of $C^{\prime}$ and $C^{\prime \prime}$ in $\mathcal{N}^{\prime}$ is equal to the order of vanishing of $\varphi_{0 \alpha^{\prime}}-\varphi_{0 \alpha^{\prime \prime}}$.

Proof of Lemma 4.1. To compute this intersection number, note that when the balls in $\mathcal{N}^{\prime}$ have small radius, the local intersection numbers can be computed with the help of the power series expansion in the third line of (1.4). In this regard, it is probably most convenient to consider the expansion in (1.4) in the polar coordinates $(s, \theta)$ as in (3.8). The details are left to the reader except for the following four remarks: First, each pair $\left(\varphi_{0 \alpha^{\prime}}, v_{0 \alpha^{\prime}}\right)$ from (1.4)'s data set has an expansion whose first term is a non-zero, real multiple of the right hand side of (3.8) for some choice of non-negative integer $q$. Second, the difference $\left(\varphi_{0 \alpha^{\prime}}-\varphi_{0 \alpha^{\prime \prime}}, v_{0 \alpha^{\prime}}-v_{0 \alpha^{\prime \prime}}\right)$, between any two such pairs also has an expansion whose first term is a non-zero, real multiple of the right hand side of (3.8). Third, one need only know the first non-zero term of this difference to compute the required local algebraic intersection numbers. Finally, the latter computation is completely straightforward.

b) The closure of $\mathcal{M}_{b, k+1}$ in $\mathcal{M}_{b, k}$. To prove that $\mathcal{M}_{b, k+1} \subset \mathcal{M}_{b, k}$ is closed, consider a sequence $\left\{C_{i}\right\} \subset \mathcal{M}_{b, k+1}-\mathcal{M}_{b, k+2}$ which converges in $\mathcal{M}_{b}$. Let $C$ denote the limit subvariety. This subvariety is in $\mathcal{M}_{b, k(C)}$ and one must show that $k(C) \geq$ 
$k+1$. The argument for this follows in twelve steps.

Step 1: Fix $\varepsilon_{0}>0$ with the following properties: First, the coordinates in (1.1) are valid on the radius $\varepsilon_{0}$ tubular neighborhood of each component of $Z$. Second, $C$ intersects each of these tubular neighborhoods as a disjoint union of embedded annuli with $z / p<<1$. Third, the coordinates $\left(t, u \equiv(2 f)^{1 / 2}\right)$ restrict to these same parts of $C$ as local coordinates. Let $\mathcal{N}$ denote the closed, radius $\varepsilon_{0} / 100$ tubular neighborhood of $Z$.

Let $C_{0}$ and $\sigma: C_{0} \rightarrow C$ be as described in Subsection 4a. The section over $\partial C_{0}$ of $\sigma^{*} N$ from Step 4 in Section $2 \mathrm{~b}$ can be chosen so that it extends with no zeros over $\mathcal{N}$, and so that corresponding push-off $C^{\prime}$ of $C$ has only positive local intersections with $C$ in the radius $\varepsilon$ tubular neighborhood of $Z$, and so that these occur only in a disjoint union, $\mathcal{N}^{\prime}$, of very small balls about the points in $Z$ where a pair from (1.4)'s data $\left\{\varphi_{0 \alpha}\right\}$ have coincident values.

With the preceding understood, the last two terms in the expression on the right hand side of (2.2) can be evaluated by restricting attention solely to the compliment of $\sigma^{-1}$ (int $\left.(\mathcal{N})\right)$ on $C_{0}$. Meanwhile, the contribution of the first term on this same side of (2.2) is a sum of two parts, one from this same compliment of $\sigma^{-1}(\operatorname{int}(\mathcal{N}))$, and one from $\mathcal{N}^{\prime}$.

Step 2: Now consider the sequence $\left\{C_{i}\right\}$. It follows from Lemmas 3.4 and 3.7 that for $i$ large, each $C_{i}$ intersects the domain $\mathcal{N}-\mathcal{N}^{\prime}$ as an embedded, push-off $C$. Thus, when $i$ is large, the given section of $C^{\prime}$ s normal bundle over $\mathcal{N}$ defines a corresponding push-off of each $C_{i}$ on the compliment of $\mathcal{N}^{\prime}$. This results in a decomposition of each term of the $C_{i}$ version of the right hand side of (2.2) into a sum of two contributions, one from the compliment of $\mathcal{N}^{\prime}$ and the other from $\mathcal{N}^{\prime}$.

Given the preceding, use Proposition 2.2 to draw the following conclusion:

When the index $i$ is large, then the contributions from the compliment of

$\mathcal{N}^{\prime}$ to each of the first two terms of the $C_{i}$ and $C$ versions of the right hand side of (2.2) are equal.

Step 3: Each $C_{i}$ has its corresponding smooth model $C_{i 0}$ with map $\sigma_{i}$. In this regard, note that the complex structure on $C_{i 0}$ is uniquely defined by $\sigma_{i}$. Moreover, on $\sigma_{i}^{-1}\left(\mathcal{N}-\mathcal{N}^{\prime}\right)$, each $C_{i 0}$ is diffeomorphic to $\sigma^{-1}\left(\mathcal{N}-\mathcal{N}^{\prime}\right) \subset C$ via a map $\lambda_{i}$ to $C$ which makes $\left\{\sigma_{i}\left(\lambda_{i}^{-1}(\cdot)\right)\right\}$ converge to $\sigma$ in the $C^{\infty}$ topology. In particular, this implies that the $\lambda_{i}$-induced complex structures on $\sigma^{-1}\left(\mathcal{N}-\mathcal{N}^{\prime}\right)$ converge to the complex structure on $C$. Now, this does not imply that the complex structure over the remainder of $C_{i}$ converge to those on $C$, although it would if the genus of the compliment of $\sigma_{i}^{-1}\left(\mathcal{N}^{\prime}\right)$ in $C_{i 0}$ was the same as that of $C_{0}$. Even so, standard results about complex structures on surfaces imply that the genus of the part of $C_{i 0}$ under consideration is no less than the genus of $C_{0}$. That is:

The contribution to $\chi\left(C_{i 0}\right)$ from the compliment of $\mathcal{N}^{\prime}$ is no greater than $\chi\left(C_{0}\right)$.

Equations (4.1) and (4.2) imply that the contribution from the compliment of $\mathcal{N}^{\prime}$ to $k\left(C_{i}\right)$ is no greater than the contribution from this same compliment to $k(C)$. In fact, the argument shows the following:

The contribution to $k\left(C_{i}\right)$ from the compliment of $\mathcal{N}^{\prime}$ is, for large $i$, strictly less than the corresponding contribution to $k(C)$ unless the compliment of $\sigma_{i}^{-1}\left(\mathcal{N}^{\prime}\right)$ in $C_{i 0}$ is diffeomorphic to that of $\sigma^{-1}\left(\mathcal{N}^{\prime}\right)$ in $C_{0}$. 
Step 4: Meanwhile, the contribution to $k\left(C_{i}\right)$ from a ball component $\mathcal{B} \subset \mathcal{N}^{\prime}$ is no greater than $\mathcal{B}^{\prime}$ s contribution to $k(C)$. In fact, one has:

The contribution to $k\left(C_{i}\right)$ from a component $\mathcal{B} \subset \mathcal{N}^{\prime}$ is no greater than $\mathcal{B}^{\prime} s$ contribution to $k(C)$; and the former is strictly less than the latter if, on $\sigma_{i}^{-1}(\mathcal{B})$, the composition of $\sigma_{i}$ with projection to the $t$-u plane has critical $(4.4)$ points.

The fact that $k\left(C_{i}\right) \leq k(C)$ for large index $i$ follows directly from (4.4) and (4.3). As previously remarked, the claim that $\mathcal{M}_{b, k}$ is closed follows from this last conclusion.

Step 5: The remaining steps in this subsection are occupied with the justification of $(4.4)$. This step starts the analysis by considering $\mathcal{B}^{\prime}$ s contribution to $k(C)$. In this regard, remember that the contribution to $k(C)$ from $\mathcal{B}$ occurs solely in the $C \bullet C$ term in (2.2). Indeed, let $o \in Z$ denote the center of this ball, and then reintroduce the data $\left\{\varphi_{0 \alpha}\right\}$ for $C$ as defined near $o$ using (1.5). By assumption, there exists a 2-element subset $\left\{\varphi_{0 \alpha^{\prime}}, \varphi_{0 \alpha^{\prime \prime}}\right\} \subset\left\{\varphi_{0 \alpha}\right\}$ whose values coincide at the point $o$. And, with this understood, the contribution, $(C \bullet C)_{o}$ to $C \bullet C$ from the ball centered at $o$ is obtained by summing over all such 2-element subsets the degree of vanishing at $o$ of the corresponding difference $\varphi_{o \alpha^{\prime}}-\varphi_{0 \alpha^{\prime \prime}}$.

The number $(C \bullet C)_{o}$ can be computed differently. Although slightly convoluted, the computation of $(C \bullet C)_{o}$ given below proves useful as it uses data from the boundary of $\mathcal{B}$ instead directly from $o$. In any event, the new computation requires a return to the milieu of the proof of Lemma 3.8 in Section 3h. As in Section 3h, the discussion can be simplified without loss of generality by assuming that all of the $\varphi_{0 \alpha}$ coincide at the point $o$ in question.

With the preceding now understood, re-introduce from the proof of Lemma 3.8 the $u>0$ half disk $D$ centered at 0 in the $t$ - $u$ plane. Take the radius $\varepsilon$ of $D$ small, so that $\left.C\right|_{D}$ lies in the ball $\mathcal{B}$ from $\mathcal{N}^{\prime}$ about the point $o$.

Also, re-introduce $\underline{C}$ to denote the metric closure $\left.C\right|_{D}$ and then $\pi^{*} \underline{C}-\underline{C}$. The later space consists of $p(p-1)$ copies of $D$ with the projection to the $t$ - $u$ plane providing this identification. Likewise, re-introduce the oriented graph $\Gamma$ as in Step 2 of the proof of Lemma 3.8. If the diameter $\varepsilon$ is small, then it follows from Lemma A.5 in [T2] that $\Gamma$ consists of a union of slightly perturbed straight arcs where each arc has one endpoint over the origin in $D$, and the other endpoints lie in the $t^{2}+u^{2}=\varepsilon^{2}$ boundary of $\pi^{*} \underline{C}-\underline{C}$. In this regard, note that two such arcs intersect (if at all) only at the point over the origin.

The number of arcs in $\Gamma$ determine $(C \bullet C)_{0}$ and vice versa. To see that such is the case, it proves convenient to introduce a subgraph $\Gamma^{0} \subset \Gamma$ which is obtained by deleting the set of $u=0$ edges from $\Gamma$ on which $t<0$ and $\operatorname{re}(\triangle)>0$. Now, let $\aleph$ denote the set of edges $e \in \Gamma^{0}$ on which $\operatorname{re}(\triangle)>0$. Here is the point:

There are exactly $(C \bullet C)_{0}$ elements in $\aleph$.

To prove (4.5), let $D^{\prime}$ and $D^{\prime \prime}$ denote the two components of $\pi^{*} \underline{C}-\underline{C}$ that correspond to a given, unordered, 2-element subset $\left\{\alpha^{\prime}, \alpha^{\prime \prime}\right\}$ of $\{1, \cdots, p\}$. Then both $\Gamma \cap D^{\prime}$ and $\Gamma \cap D^{\prime \prime}$ are comprised of $m+1$ arcs, where $m$ is the degree of vanishing of $\varphi_{0 a^{\prime}}-\varphi_{0 \alpha^{\prime \prime}}$ at $t=0$. (In both cases, two of the arcs lie where $u=0$.) The total number of arcs in $\Gamma \cap\left(D^{\prime} \cup D^{\prime \prime}\right)$ which have re $(\triangle)>0$ is $m+1$ since those with re $(\triangle)<0$ in $D^{\prime}$ have $\operatorname{re}(\triangle)>0$ in $D^{\prime \prime}$ and vice-versa. On the other hand, precisely 
one of these arcs is deleted from $\Gamma$ to make $\Gamma^{0}$. Thus, each of the two element subsets of $\{1, \cdots p\}$ contributes the same number of elements to $\aleph$ as it does to $(C \bullet C)_{0}$.

Step 6: Now consider, for large index $i$, the corresponding space $\pi^{*} \underline{C}_{i}-\underline{C}_{i}$ and the corresponding oriented graph $\Gamma_{i}$. The point of introducing $\Gamma_{i}$ is that the contribution to $k\left(C_{i}\right)$ from $\mathcal{B}$ can be determined with the help of $\Gamma_{i}$. However, this ultimate goal requires a digression in order to describe $\Gamma_{i}$. This digression occupies Steps 6-9.

The digression starts in this step with a description of $\Gamma_{i}$ near the boundary of $\pi^{*} \underline{C}_{i}-\underline{C}_{i}$ where $t^{2}+u^{2}=\varepsilon^{2}$. In particular, the proof of Lemma 3.8 noted that $\Gamma_{i}$ near the $t^{2}+u^{2}=\varepsilon^{2}$ boundary of $\pi^{*} \underline{C}_{i}-\underline{C}_{i}$ consists of exactly $2 \cdot(C \bullet C)_{0}+p(p-1)$ disjoint, slightly perturbed arcs, with each ending on this boundary. Of these, precisely $2 p(p-1)$ project to the $u=0$ line segment in $D$. The point here is that near this boundary $\pi^{*} \underline{C}_{i}-\underline{C}_{i}$, the arcs which comprises $\Gamma_{i}$ are in 1-1 correspondence with those which comprise $\Gamma$. Indeed, each arc in $\Gamma_{i}$ is very close (when $i$ is large) to a like oriented arc in $\Gamma$ and vice-versa.

Step 7: Other vertices of $\Gamma_{i}$ lie either on the $u=0$ line or are critical points of the function $\underline{b}$. Lemma A.4 in [T2] describes $\Gamma_{i}$ near the latter: There are an even number greater than 2 of incident edges, where half are oriented inward and half outward (these orientations alternate as the vertex is circled.)

Step 8: An edge of $\Gamma_{i}$ can limit to a point in $\underline{C}_{i} \subset \pi^{*} \underline{C}_{i}$. Since $\triangle_{i}$ vanishes on $\underline{C}_{i}$ this function is not bounded away from zero on such an edge. In any event, Step 5 in Part b of the Appendix to [T2] describes how $\Gamma_{i}$ intersects a neighborhood of a point in $\underline{C}_{i}$ : There is a non-zero and even number of edges in this neighborhood which approach the given point in $\underline{C}_{i}$, with half having $\operatorname{re}\left(\triangle_{i}\right)>0$ and half having $\operatorname{re}\left(\triangle_{i}\right)<0$.

Here is a pertinent example: If the point in $\underline{C}_{i}$ under consideration is an immersion point where $(t, u)$ are good coordinates on each sheet, then there will be four incident edges of $\Gamma_{i}$ with exactly two having $\operatorname{re}\left(\triangle_{i}\right)>0$.

Here is a second pertinent example: There are precisely two edges of $\Gamma_{i}$ near a manifold point of $\underline{C}_{i}$ where the projection $\pi$ to the $t$ - $u$ plane is critical. And, precisely one of the latter has $\operatorname{re}\left(\triangle_{i}\right)>0$.

Step 9: Very close to the $u=0$ line, $\pi^{*} \underline{C}_{i}-\underline{C}_{i}$ consists of $p(p-1)$ strips, each projecting to $D$ as $\left\{(t, u): t^{2}+u^{2} \leq \varepsilon\right.$ and $\left.0 \leq u \leq \delta\right\}$. Here $\delta$ is positive, but $\delta<<\varepsilon$. (Note that the size of $\delta$ will depend on the index $i$.) Indeed, when $\delta$ is small, then the analysis of $\Gamma_{i}$ where $u<\delta$ proceeds as with $\Gamma$ in Step 4 above, and finds that $\Gamma_{i}$ intersects each such strip as a union of almost straight arcs. Each arc where $u$ is not identically zero will have one endpoint where $u=\delta$ and the other where $u=0$. At a $u=0$ endpoint, the complex function $\triangle_{i}$ (the restriction to $\pi^{*} \underline{C}_{i}$ of $\eta_{-}-\eta_{+}$) either vanishes or not. In this regard, remember that the $u=0$ arc endpoints where $\triangle_{i}=0$ correspond to the triples $\left(t\left\{\varphi_{i, 0 \alpha^{\prime}}, \varphi_{i, 0 \alpha^{\prime \prime}}\right\}\right)$ where $t \in(-\varepsilon, \varepsilon)$ and where $\left\{\varphi_{i, 0 \alpha^{\prime}}, \varphi_{i, 0 \alpha^{\prime \prime}}\right\}$ is a pair from the index $i$ version of the data set from (1.5) with coincident values at $t$. If the degree of vanishing of the difference, $\varphi_{i, 0 \alpha^{\prime}}-\varphi_{i, 0 \alpha^{\prime \prime}}$ is $m$, then this vertex has $m+1$ incident edges with two lying on the $u=0$ locus.

Next, recall from Lemma A.4 in Part b of the Appendix in [T2] that the edges of $\Gamma_{i}$ are oriented by the restriction of the 1 -form $d\left(\operatorname{re}\left(\triangle_{i}\right)\right)$. Moreover, it follows from 
Lemma A.5 in this same reference that each $\triangle_{i}=0$ vertex on the $u=0$ line has at least one incident edge with $\mathrm{re}\left(\triangle_{i}\right)>0$. In fact, according to Lemma A.5 in [T2], the inward and outward pointing edges which are incident on this vertex alternate when travelling around a small half circle which starts on the $u=0$ line above the vertex (as measured by $t$ ) and ends on the $u=0$ line below the same vertex. Thus, if the number of such edges is even, then there are equal numbers of inward pointing and outward pointing edges. If there is an odd number of incident edges, then the numbers of inward pointing and outward pointing edges differ by one. (As $\triangle_{i}$ vanishes on the vertex in question, outward pointing edges have $\operatorname{re}\left(\triangle_{i}\right)>0$ and inward pointing ones have $\operatorname{re}\left(\triangle_{i}\right)<0$.) Furthermore

- If the number of incident edges is even and if there is an outward pointing incident edge where $u=0$ and $|t|<|t(v)|$, then there is also inward pointing edge with $u=0$ and $|t|>|t(v)|$.

- If the number of incident edges is odd, then either both $u=0$ edges are inward pointing, or both are outward pointing.

Meanwhile, the arguments for Lemma A.5 in [T2] work just as well for edge endpoints on the $u=0$ line where $\operatorname{re}\left(\triangle_{i}\right)$ is not equal to zero. In particular, the description of the incident edges from the preceding paragraph holds at these vertices as well.

Step 10: With the digression now at an end, it is time to get to the point about $\Gamma_{i}$. For this purpose, introduce $\Gamma_{i}^{0} \subset \Gamma_{i}$ to denote a certain subgraph which is obtained by deleting appropriate edges where $u=0$. To be precise, delete a $u=0$ edge $e$ if $\operatorname{re}\left(\triangle_{i}\right)>0$ on $e$, and if $t$ is negative at $e$ 's starting vertex and increases along $e$. Likewise, delete a $u=0$ edge $e$ if $\operatorname{re}\left(\triangle_{i}\right)>0$ on $e$ and if $t$ is positive at $e$ 's starting vertex and decreases along $e$.

With $\Gamma_{i}^{0}$ understood, let $\aleph_{i}$ denote the set of edges of $\Gamma_{i}^{0}$ on which $\operatorname{re}\left(\triangle_{i}\right)>0$, but not bounded away from zero. Now consider:

Lemma 4.2. When the index $i$ is large, then $\aleph$ has at most $(C \bullet C)_{0}$ elements.

This lemma is proved below, so accept it for the moment as it is the key to the proof (4.4).

Here is a simple case which exhibits the lemma's application:

- Assume that the map $\sigma_{i}$ immerses $\sigma_{i}^{-1}(\mathcal{B})$ in $X$.

Under the preceding assumption, the contribution from $\mathcal{B}$ to the $C_{i}$ version of (2.2) is a sum of the following two terms.

- Twice the number of double points of $\sigma_{i}$ on $\sigma_{i}^{-1}(\mathcal{B})$.

- The sum of integer weights indexed by the triples $\left(\tau,\left\{\varphi_{i, o \alpha^{\prime}}, \varphi_{i, 0 \alpha^{\prime \prime}}\right\}\right)$ where $\tau$ lies on the $u=0$ line in the $t$ - $u$ plane where $|t|<\varepsilon$, and where $\left\{\varphi_{i, 0 \alpha^{\prime}}, \varphi_{i, 0 \alpha^{\prime \prime}}\right\}$ is an unordered, 2-element subset from $\left\{\varphi_{i, 0 \alpha}\right\}$ whose values coincide at $\tau$. The weight for such a triple is the degree of vanishing of $\varphi_{i, 0 \alpha^{\prime}}-\varphi_{i, 0 \alpha^{\prime \prime}} \cdot$ (4.8)

With (4.8) understood, note that Steps 8 and 9 have the following consequence: The sum of the terms in (4.8) is never greater than the number of elements in $\aleph_{i}$. Moreover, this sum is strictly less than the number of elements in $\aleph_{i}$ if the composition of $\sigma_{i}$ with the projection to the $t$ - $u$ plane has critical points. Thus, under the assumption 
that $\sigma_{i}$ immerses $\sigma_{i}^{-1}(\mathcal{B})$, Lemma 4.2 guarantees (5.4).

The next step explains why Lemma 4.2 implies (4.4) without the constraint in (4.7).

Step 11: Here is how to go from Lemma 4.2 to (4.4) in the general case. Take $\sigma>0$ but very small (as in Step 8 above) and perturb $\sigma_{i}$ where $\sigma_{i}^{*} u>\delta$ on $\sigma_{i}^{-1}(\mathcal{B})$ where $u>\delta$ (with $\delta$ very small) to make the result a pseudoholomorphic immersion. The construction of such a perturbation is standard using the analysis from either Section 5, below, or from [MS]. Moreover, the perturbation can be made so as to be close to the original where $\sigma_{i}^{*} u \geq 2 \delta$ in any given topology. Then, by employing a bump function, the perturbation can be melded to the original where $\delta \leq \sigma_{i}^{*} u \leq 2 \delta$ to give a map, $\sigma_{i}^{\prime}$ from $\sigma_{i}^{-1}(\mathcal{B})$ with the following properties: First, the latter is pseudoholomorphic where $\sigma_{i}^{*} u \geq 2 \delta$. Second, it agrees with $\sigma_{i}$ where $\sigma_{i}^{*} u \leq \delta$. Third, it is close to $\sigma_{i}$ on $\sigma_{i}^{-1}(\mathcal{B})$ in any $C^{k}$ topology given a priori.

Here are the reasons for considering such a map $\sigma_{i}^{\prime}$ : If the $\sigma_{i}^{\prime}$ is close to $\sigma_{i}$ as measured by the $C^{1}$ topology, then the following four points can be assumed

- The contribution to $k\left(C_{i}\right)$ from $\mathcal{B}$ can be computed using $\sigma_{i}^{\prime}$ instead of $\sigma_{i}$.

- The pull-back of the function $t$ by $\sigma_{i}^{\prime}$ will have critical points if $\sigma_{i}$ pulls $t$ back with critical points.

- The corresponding oriented graph $\Gamma_{i}^{\prime}$ will be a slight push-off of $\Gamma_{i}$ where $\sigma_{i}^{*} u \leq 2 \delta$ and near where $\sigma_{i}^{*}\left(t^{2}+u^{2}\right)=\varepsilon^{2}$.

- The corresponding $\aleph_{i}^{\prime}$ can be defined even though $\sigma_{i}^{\prime}$ is not quite pseudoholomorphic everywhere.

Indeed, the first point above follows directly from the definition of $k(\cdot)$. The second point in (4.9) follows from (A.6) in [T2]. The third point follows from the discussion in Step 9 above, and then the final point follows from the third point.

With (4.9) understood, the argument in the previous step as applied to the resulting $\aleph_{i}^{\prime}$ shows that the contribution to $k\left(C_{i}\right)$ from $\mathcal{B}$ is strictly less than $\mathcal{B}^{\prime} s$ contribution to $k(C)$ and thus verifies (4.4) in the general case.

Step 12: This step contains the

Proof of Lemma 4.2. Introduce, as in (A.28) of [T2], the notion of an oriented, graphical path. In the present context, this is an embedded, oriented path in $\Gamma_{i}^{0}$ which is a connected union of edges and vertices, and which traverses edges only in the direction of their orientation by $d\left(\operatorname{re}\left(\triangle_{i}\right)\right)$. With this notion understood, Lemma 4.2 follows directly from the discussion in Step 6 above and the following assertion:

Each $e \in \aleph_{i}$ labels on oriented, graphical path $\psi_{e}$ in $\Gamma_{i}^{0}$ whose first edge is $e$, and which ends where $t^{2}+u^{2}=\varepsilon$. Moreover, if $e \neq e^{\prime}$, then intersections between $\psi_{e}$ and $\psi_{e^{\prime}}$ occur, if at all, only at verticies of $\Gamma_{i}^{0}$ where $\operatorname{re}\left(\triangle_{i}\right)>0$.

Here is how to build the set $\left\{\psi_{e}: e \in \aleph_{i}\right\}$ : Start with an edge $e \in \aleph_{i}$ and use the arguments for Lemma A.6 in [T2] to construct the required oriented, graphical path $\psi_{e}$. The point to Lemma A.6 in [T2] is that there are no obstructions to successively adding edges to an initial edge $e$ until the $t^{2}+u^{2}=\varepsilon^{2}$ half-circle is reached; there are new edges to extend $\psi_{e}$ past any vertex of $\Gamma_{i}^{0}$. In this regard, the fact that $\operatorname{re}\left(\triangle_{i}\right)$ 
increases along $\psi_{e}$ insures that $\psi_{e}$ is embedded and never returns to $\aleph_{i}$. (Use (4.5) when applying the arguments from Lemma A.6 in [T2] to extend $\psi_{e}$ past any $u=0$ vertex of $\Gamma_{i}^{0}$.)

To construct $\psi_{e^{\prime}}$ for other $e^{\prime} \in \aleph_{i}$, employ the inductive strategy from the proof of Lemma A.6 in [T2]. To summarize, suppose that $\psi_{e}$ has been constructed for all $e$ in a subset $\aleph_{i}^{\prime} \subset \aleph_{i}$. Suppose that this collection $\left\{\psi_{e}: e \in \aleph_{i}^{\prime}\right\}$ satisfies the following criteria:

- Each $\psi_{e}$ is an oriented, graphical path whose starting edge is $e$ and whose ending vertex is on the $t^{2}+u^{2}=\varepsilon^{2}$ half-circle.

- The collection $\left\{\psi_{e}\right\}$ is pairwise disjoint except possibly for the sharing of vertices of $\Gamma_{i}^{0}$ where $\operatorname{re}\left(\triangle_{i}\right)>0$.

Let $\Gamma_{i}^{0^{\prime}}$ denote the compliment in $\Gamma_{i}^{0}$ of the union of the edges of the graphical paths $\left\{\psi_{e^{\prime}} \in \aleph_{i}^{\prime}\right\}$. If $e^{\prime} \in \aleph_{i}-\aleph_{i}^{\prime}$, then the construction of $\psi_{e}$ just described for the first choice of $e \in \aleph_{i}$ can now be repeated essentially verbatim but with $\Gamma_{i}^{0^{\prime}}$ replacing $\Gamma_{i}^{0}$. The result is a graphical $\psi_{e^{\prime}}$ which obeys the first point in (4.11) and is disjoint from $\left\{\psi_{e}: e \in \aleph_{i}^{\prime}\right\}$ except possibly at vertices of $\Gamma_{i}$ with $\operatorname{re}\left(\triangle_{i}\right)>0$. These last points insure that the induction argument can be continued until all edges from $\aleph_{i}$ are accounted for.

c) The Proof of Proposition 2.7. Proposition 2.7 follows as an immediate corollary from Lemma 4.3, below, and Lemma 3.7; the argument is the same as that used in the previous section for Proposition 2.10.

Lemma 4.3. Let $C \in \mathcal{M}_{b, k}-\mathcal{M}_{b, k+1}$. Then $C$ has a neighborhood $\mathcal{U}_{0} \subset \mathcal{M}_{b, k}$ and each point in $Z$ has a neighborhood $B$ and an integer $p$ such that $C^{\prime} \in \mathcal{U}_{0}$ intersects $B$ as a union of $p$ irreducible components.

Proof of Lemma 4.3. Let $Z^{\prime} \subset Z$ be a component. As in (1.4), there is an integer $p \geq 0$ such that each point in $Z^{\prime}$ has a ball neighborhood which intersects $C$ in precisely $p$ disjoint components. Using (4.4), an argument via reduction ad absurdum finds a neighborhood $\mathcal{U}_{0} \subset \mathcal{M}_{b, k}-\mathcal{M}_{b, k+1}$ of $C$ and a tubular neighborhood $U \subset X$ of $Z^{\prime}$ such that each $C^{\prime} \in \mathcal{U}_{0}$ intersects $U$ as a multi-valued graph over the $t$ - $u$ plane. This directly implies Lemma 4.3.

5. Fredholm operators. The purpose of this section is to present the proof of Proposition 2.6 and then that of Proposition 2.8. Note that the proof of Proposition 2.6 is carried out first under the restricted assumption that $C$ is the image of an immersion; the latter arguments are then generalized to handle all cases. The motivation for this is simply that the complications which arise from $C$ 's lack of compactness can be separated from those which arise from non-immersion points. Moreover, the former are novel to the story here, while the latter appear already in the case where $X$ is symplectic.

a) The definition of $D_{c}$. Start with a given $C \in \mathcal{M}_{b}$ which is the image of an immersion into $X-Z$. Then $C$ has a normal bundle, $N \rightarrow C$, which inherits the structure of a complex line bundle from the almost complex structure $J$. Moreover, the 
Riemannian metric gives $N$ a hermitian structure and thus a holomorphic structure.

There is a tubular neighborhood $N_{0} \subset N$ of the zero section of $N$ with an exponential map $e: N_{0} \rightarrow X-Z$ which maps $N_{0}$ onto a neighborhood of $C$ and sends the zero section onto $C$. In particular, if $K \subset C$ is compact, then there exists some $\delta>0$ such that $\left.N_{0}\right|_{K}$ contains the radius $\delta$ disk bundle in $N$. Note that $e$ can be chosen so as to map each fiber of $N_{0}$ onto a pseudoholomorphic disk. (See, e.g. Lemma 2.2 in [T6].)

Via composition with $e$, a section $s$ of $\left.N_{0}\right|_{K}$ defines a deformation of $K$. The condition that this deformation be pseudoholomorphic translates into a certain nonlinear differential equation for the sections $s$. The latter has the schematic form

$$
\bar{\partial} s+R_{0}(s)+R_{1}(s, \nabla s)=0
$$

Here, $R_{0}$ is an $\mathbb{R}$-linear, fiber preserving map from $N_{0}$ to $N \otimes T^{0,1} C$, while $R_{1}$ is a nonlinear, fiber preserving, real analytic map from $N_{0} \otimes\left(N_{0} \otimes T^{*} C\right)$ to $N \otimes T^{0,1} C$. Note that, $\left|R_{0}\right|$ is bounded by a $K$-dependent constant, while $\left|R_{1}(a, b)\right| \leq \zeta_{K}|a|(|a|+|b|)$, where $\zeta_{K}$ depends on $K$ as indicated. (The $\bar{\partial}$ operator in (5.1) and the covariant derivative $\nabla$ are defined in the usual way from the complex hermitian structure on N.)

The linearization of (5.1) defines the differential operator $D_{C}$ over $K$, namely

$$
D_{C} s=\bar{\partial} s+R_{0}(s) \text {. }
$$

With $D_{c}$ understood, consider now its domain and range: The domain $\mathfrak{D}$ is defined to be the closure of the space of smooth sections of $N \rightarrow C$ using the norm

$$
\|s\|_{\mathfrak{D}}^{2} \equiv \int_{C}\left(|\nabla s|^{2}+|\omega|^{-2}|s|^{2}\right) \omega
$$

Meanwhile, the range $\mathcal{R}$ for $D_{C}$ is the closure of the space of smooth sections of $N \otimes T^{0,1} C$ using the norm $\|s\|_{\mathcal{R}}^{2} \equiv \int_{c}|s|^{2} \omega$. In both cases, the fiber norms on $N \otimes T^{*} C$ are defined using the metric which is induced from the metric on $X$. Note that the obvious polarizations of the norms here give inner products that make both $\mathfrak{D}$ and $\mathcal{R}$ Hilbert spaces.

b) Outline of the proof of Proposition 2.6 for immersed $C$. Let $C$ again denote an immersed element of $\mathcal{M}_{b}$. With $D_{C}$ understood, the initial arguments for Proposition 2.6 verify that the operator $D_{C}$ extends to $\mathfrak{D}$ as a Fredholm map into $\mathcal{R}$. With this task accomplished, the next part of the proof follows a well worn path: This path begins by finding the ball $B \subset$ kernel $\left(D_{C}\right)$ with its map $f: B \rightarrow$ cokernel $\left(D_{C}\right)$. This is done in the usual way: The implicit function theorem is first used to find

- $A$ ball $B \subset$ kernel $\left(D_{C}\right)$.

- A smooth map, $s_{1}$ from $B$ to the orthogonal compliment of kernel $\left(D_{C}\right)$ in $\mathfrak{D}$ which is such that

a) $s_{1}(0)=0$.

b) $s \equiv s_{0}+s_{1}\left(s_{0}\right)$ solves all of (4.3) save, perhaps, its projection onto the cokernel of $D_{C}$.

The map $f$ in the statement of Proposition 2.6 is then obtained by projecting (5.3) (as an $\mathcal{R}$-valued function on $B$ ) onto the cokernel of $D_{C}$. Meanwhile, the map $\mathcal{F}$ in 
Proposition 2.6 sends $s \in B$ to the image of the composition of $s$ (as a section of $N$ ) with the exponential map $e$.

The final part of the proof of Proposition 2.6 verifies that when $C \in \mathcal{M}_{b, k}$ $\mathcal{M}_{b, k+1}$, then the ball $B$ can be chosen so that $\mathcal{F}$ as just defined maps $f^{-1}(0)$ homeomorphically onto a neighborhood of $C$ in $\mathcal{M}_{b, k}$.

The formal aspects of the proof just outlined are completely standard (see, e.g. [MS]). However, the non-compactness of $C$ lends some novelty to the implimentation of the standard arguments. Needless to say, the novel part of the analysis takes place near $Z$.

c) Proof of Proposition 2.6 when $C$ is immersed. The proof is broken into ten steps, where the first six are devoted to the proof that $D_{C}$ is Fredholm when $C \in \mathcal{M}_{b}$ is immersed in $X-Z$.

Step 1: This first step sets the stage by summarizing various relevant properties of $C$. To begin, recall that an $\varepsilon>0$ exists such that the $r \leq \varepsilon$ region of $C$ satisfies the conclusions of Lemma 3.5 near any given component $Z^{\prime} \subset \bar{Z}$. Moreover, in this region, the functions $t$ and $u$ restrict as local coordinates so that $C$ is represented locally as a $p$-sheeted graph $\left(t, u,\left(\varphi_{\alpha}(t, u), v_{\alpha}(t, u)_{1 \leq \alpha \leq p}\right)\right.$. Here the set $\left\{\left(\varphi_{\alpha}, v_{\alpha}\right)\right\}$ can be ordered locally, but the ordering may be permuted upon cycling around $Z^{\prime}$. Also, if the $z$ axis is unoriented as a line bundle over $Z^{\prime}$, then cycling around $Z^{\prime}$ multiplies each pair from the set $\left\{\left(\varphi_{\alpha}, v_{\alpha}\right)\right\}$ by -1 . In any event, $\varphi_{\alpha}(t, u)=\varphi_{0 \alpha}(t)+\mathcal{O}\left(u^{2}\right)$ while $v_{\alpha}(t, u)=4^{-1}\left(\partial_{t} \varphi_{0 \alpha}\right) u+\mathcal{O}\left(u^{3}\right)$; and both of these locally defined functions have analytic dependence on $t$ and $u$.

As just remarked, there may be some permutation of the labels of $\left(\varphi_{\alpha}, v_{\alpha}\right)$ and multiplication by -1 around $Z^{\prime}$. Fix attention on a non-empty subset of the label set $\{1, \cdots, p\}$ on which the resulting $\mathbb{Z}$ action is transitive. One can assume that this subset has labels $\{1, \cdots, q\}$. This subset corresponds to an annular component, $A$, of the $r \leq \varepsilon$ region of $C$, whereby the projection to the $(t, u)$ plane is a $q$ to 1 covering map. Thus, the metric closure of $A$ is parameterized by coordinates $(\tau, u) \in$ $\mathbb{R} /(2 \pi q \mathbb{Z}) \times[0, \varepsilon]$. Here, the projection from the $u=0$ boundary to $Z$ is the standard $q$ to 1 map from $\mathbb{R} /(2 \pi q \mathbb{Z})$ to $\mathbb{R} /(2 \pi \mathbb{Z})$.

Meanwhile, for $\varepsilon$ small, the pseudoholomorphic submanifolds given by a pair of numbers $\left(c, c^{\prime}\right) \in \mathbb{R} /(2 \pi \mathbb{Z}) \times\left(0, \varepsilon^{2} / 8\right)$ by setting $t=c$ and $f=c^{\prime}$ intersect the given annular component $A \subset C$ transversally near $Z^{\prime}$. Thus, a section of the normal bundle of $A$ is specified by a pair $\psi \equiv(\alpha, \beta)$ of sections over $\mathbb{R} /(2 \pi q \mathbb{Z}) \times(0, \varepsilon]$ of a certain real line bundle over $\mathbb{R} /(2 \pi q \mathbb{Z}) \times[0, \varepsilon]$. Here, the line bundle in question is naturally isomorphic to the restriction to $A$ of the $z$-axis line bundle (which is defined over a tubular neighborhood in $X$ of each component of $Z)$. In particular, $(\alpha, \beta)$ are not bonafide functions if and only if the $z$-axis line bundle is unorientable and $q$ is odd. In any case, the implicit exponential map sends $\left.((\tau, u),(\alpha, \beta)) \in N\right|_{A}$ to the point in the manifold $X$ with coordinates $(\tau, u, \varphi(\tau, u)+\alpha, v(\tau, u)+\beta)$.

With this last point understood, the norm $\|\cdot\|_{\mathfrak{D}}$ in (5.3) for a section with support on $A$ is equivalent to the norm for the pair $\psi \equiv(\alpha, \beta)$ whose square is

$$
\|\psi\|_{*: *}^{2}=\int_{A}\left(|d \psi|^{2}+u^{-2}|\psi|^{2}\right) u^{3} d \tau d u .
$$

Likewise, the norm $\|\cdot\|_{\mathcal{R}}$ in terms of these same coordinates $(\alpha, \beta)$ for the normal bundle has square $\|\cdot\|_{*}^{2}=\int_{A}|\cdot|^{2} u^{3} d \tau d u$. 
Finally, note that the operator $D_{C}$ in (5.2), when attacking a section of $\left.N\right|_{A}$, can be written in terms of the data $\psi=(\alpha, \beta)$ with the following result: $D_{C} \psi$ has two components (the real and imaginary parts of the expression in (5.2)), and they can be written as

$$
\begin{aligned}
& \text { - } \alpha_{\tau}-\beta_{u}-3 u^{-1} \beta+\kappa_{1} \alpha_{\tau}+2 \kappa_{1}^{\prime} v \varphi_{\tau} \beta \\
& \text { - } \alpha_{u}+\beta_{\tau}+\kappa_{2} \alpha_{u}+2 \kappa_{2}^{\prime} v \varphi_{u} \beta \text {. }
\end{aligned}
$$

Here, $\kappa_{1,2}$ are the functions of $v^{2}$ which appear in (3.4), while $\kappa_{1.2}^{\prime}$ denote their derivatives with respect to this variable. (Remember that $\kappa_{1,2}=\mathcal{O}\left(v^{2}\right)$ and that both are analytic functions when $v^{2}<1 / 100$.)

Step 2: This step states and proves a fundamental inequality for the operator $D_{C}$. The inequality in question is summarized by

Lemma 5.1. If the constant $\varepsilon>0$ which defines (in part) the annular region $A \subset$ $C$ is sufficiently small, then there is a constant $\zeta \geq 1$ with the following significance: Let $\psi=(\alpha, \beta)$ have support on $A$ and vanish near the $u=\varepsilon$ boundary of $A$. Suppose that $\alpha$ and $\beta$ restrict as $L_{1}^{2}$ functions to every open set in $A$ on which $u$ is bounded away from zero. Moreover, assume that both $\left\|D_{C} \psi\right\|_{*}$ and $\left\|u^{-1} \psi\right\|_{*}$ are finite. Then $\|\psi\|_{* *}$ is finite and $\|\psi\|_{* *} \leq \zeta\left\|D_{C} \psi\right\|_{*}$.

Proof of Lemma 5.1. It is sufficient to prove the inequality using smooth $\psi=$ $(\alpha, \beta)$. With this understood, fix a bump function $\chi:[0, \infty) \rightarrow[0,1]$ which is 1 near zero and 0 on $[1, \infty)$ and nowhere increasing. For the moment, also fix $\delta>0$ but with $\delta<<\varepsilon$. Let $\chi_{\delta}$ denote the function of $u$ whose value is that of $\chi$ at the point $u / \delta$.

Square the expressions in (5.6), multiply the result by $\left(1-\chi_{\delta}\right)^{2}$ and integrate over $A$ with respect to the measure $u^{3} d \tau d u$. Integration by parts finds that

$$
\begin{aligned}
\int_{A}\left(1-\chi_{\delta}\right)^{2}\left|D_{C} \psi\right|^{2} u^{3} d \tau d u \geq \int_{A}\left(1-\chi_{\delta}\right)^{2}\left(|d \psi|^{2}+3 u^{-2} \beta^{2}\right) u^{3} d \tau d u \\
-\xi \varepsilon^{2}\left(\left\|\left(1-\chi_{\delta}\right) d \psi\right\|_{*}^{2}+\left\|\left(1-\chi_{\delta}\right) u^{-1} \psi\right\|_{*}^{2}\right)+\xi\left\|\left|d \chi_{\delta}\right| \psi\right\|_{*}^{2}(5.7)
\end{aligned}
$$

Here, $\xi \geq 1$ is a constant which is independent of $\varepsilon$ if the latter is small, and independent of $\psi$. By the way, the terms with the $\varepsilon^{2}$ factors in (5.7) come from the terms in (5.6) with $\kappa_{1,2}$ and $\kappa_{1,2}^{\prime}$. Meanwhile, the last term in (5.7) comes from rewriting $\int_{u>\delta}\left(1-\chi_{\delta}\right)^{2}\left[\alpha_{u}\left(u^{3} \beta\right)_{\tau}-\alpha_{\tau}\left(u^{3} \beta\right)_{u}\right] d \tau d u$ via an application of integration by parts and then the triangle inequality.

The next observation is that the last term in (5.7) tends to zero as $\delta$ tends to zero. Indeed, to see that such is the case, note that the last term is no larger than $\zeta \int_{A \cap\{u<b\}}|\psi|^{2} u d \tau d u$. Since this same integral is finite for $\delta=\varepsilon$, the corresponding sequence of $\delta$ versions is monotonically decreasing as $\delta$ tends to zero with limit zero.

Given this last point, it follows that the $\delta \rightarrow 0$ limit in (5.7) can be taken to yield the inequality $\left\|D_{C} \psi\right\|_{*} \geq\|d \psi\|_{*}-\zeta \varepsilon\|\psi\|_{* *}$. This is not quite the required inequality since $\|\psi\|_{* *}^{2}$ is the sum of $\|d \psi\|_{*}^{2}$ and $\left\|u^{-1} \psi\right\|_{*}^{2}$. However, a simple integration by parts argument demonstrates that the latter is no greater than the former if $\psi$ vanishes near the $u=\varepsilon$ circle in $A$. Thus, the $\delta \rightarrow 0$ limit in (5.7) gives the inequality $\left\|D_{C} \psi\right\|_{*} \geq\left(2^{-1 / 2}-\xi \varepsilon\right)\|\psi\|_{* *}$. And, this last inequality verifies the assertions of the lemma.

Step 3: Lemma 5.1 has the following global statement as a corollary: 
LEMMA 5.2. There is a compact, measurable set $K \subset C$ and a constant $\zeta \geq 1$ with the following significance: If $s \in \mathfrak{D}$, then $\|s\|_{\mathfrak{D}} \leq \zeta\left(\left\|D_{C} s\right\|_{\mathcal{R}}+\left\|\chi_{K} s\right\|_{\mathcal{R}} \|\right.$ where $\chi_{K}$ denotes the characteristic function of $K$.

Proof of Lemma 5.2. Fix a smooth, non-negative function $\chi$ on $X$ which is one on some very small radius tubular neighborhood of $Z$ and which is zero on the compliment of the concentric tubular neighborhood with twice the radius. In particular, the support of the restriction of $\chi$ to $C$ should lie in the union of the annuli $A$ as described above. Then write $s=\chi s+(1-\chi) s$. A standard trick then finds $\left\|D_{C} s\right\|_{\mathcal{R}} \geq \zeta^{-1}\left(\left\|D_{C}(\mathcal{X} s)\right\|_{\mathcal{R}}+\left\|D_{C}((1-\chi) s)\right\|_{\mathcal{R}}-\|d \chi s\|_{\mathcal{R}}\right)$, where $\zeta \geq 1$ is independent of $s$. Now apply Lemma 5.1 to the term with $D_{C}(\mathcal{X} s)$ and apply a standard elliptic inequality to that with $D_{C}((1-\chi) s)$ to obtain the lemma.

Step 4: Lemma 5.2 implies that $D_{C}$ has finite dimensional kernel. This is a standard argument because the Rellich theorem implies that the map from $s \rightarrow \chi_{K} s$ from $\mathfrak{D}$ to $L^{2}(K)$ is compact when $K \subset C$ is compact and measurable. Or, one can argue as follows: As $D_{C}$ is elliptic, each $s \in \operatorname{kernel}\left(D_{C}\right)$ with $\|s\|_{\mathfrak{D}}=1$ enjoys a priori bounds for its first derivatives on an open set with compact closure in $C$. Because of this fact, an infinite dimensional kernel would imply, given $\varepsilon>0$, the existence of $s \in$ kernel $(D)$ with $\|s\|_{\mathfrak{D}}=1$ and with $|s| \leq \varepsilon$ at each point of $K$. Indeed, given any finite number of points in $K$, one could find an $s \in$ kernel $\left(D_{C}\right)$ which vanished at each. Scaling by $\mathbb{R}^{*}$ would find such an $s$ with $\|s\|_{\mathfrak{D}}=1$. Make the number of such points large and reasonably uniformly spread over $K$ and then the apriori bound on the first derivatives of $s$ would require $|s|$ to be uniformly small $(<\varepsilon)$ on the whole of $K$. Of course, if $\varepsilon$ is small, then Lemma 5.2' inequality can not hold. This contradiction forces the conclusion that kernal $\left(D_{C}\right)$ is finite dimensional.

Step 5: The purpose of this step is to describe the behavior of an element in $s \in \overline{\text { kernel }}\left(D_{C}\right)$ near $Z$. In particular, consider

LEMMA 5.3. Let $s$ be a smooth section of $C$ 's normal bundle which is annihilated by $D_{C}$ and which obeys $\int_{C}|s|^{2} w=1$. Then $s \in \mathfrak{D}$. Moreover, $s$ extends to the metric closure of $C$ in the following way: On the annular end $A \subset C$, write $s$ in terms of $(\alpha, \beta)$ as in (5.6). Then $\alpha$ and $\beta$ extend over the missing boundary, $S^{1} \times\{0\} \subset A$ to define real analytic functions of the variables $(\tau, u)$ for $\mathbb{R} /(2 \pi q \mathbb{Z}) \times[0, \varepsilon]$. In addition, $\alpha=\alpha_{0}(\tau)+\mathcal{O}\left(u^{2}\right)$ and $\beta=4^{-1} \alpha_{0}^{\prime}(\tau) u+\mathcal{O}\left(u^{3}\right)$.

Proof of Lemma 5.3. Let $B \subset \mathbb{R}^{4}$ denote the ball about the origin of radius $\varepsilon$, and interpret $u$ as the radial coordinate on $B$. In this way, $\alpha$ becomes a section, $\underline{\alpha}$, of one of the two real line bundles over $S^{1} \times(B-0)$. Meanwhile, introduce the three form $\underline{\beta} \equiv \beta u^{3} \sigma$ where $\sigma$ is the volume 3 -form on the unit sphere in $\mathbb{R}^{4}$. Note that the condition that $\int_{C}|s|^{2} \omega<\infty$ implies that both $\underline{\alpha}$ and $\underline{\beta}$ are square integrable on $S^{1} \times B$ with respect to the standard volume element.

With $\underline{\alpha}$ and $\underline{\beta}$ understood, (5.6) can be rewritten schematically as

$$
d \underline{\alpha}+* d \underline{\beta}+*(w \wedge \underline{\beta})=0 .
$$

Moreover, $d * \underline{\beta}=0$ because $\beta$ is a function only of $t$ and $u$. Here and in (5.8), the symbol * denotes the Hodge star for a certain real analytic metric on $S^{1} \times B$ which is within $\mathcal{O}\left(u^{2}\right)$ of the product flat metric; meanwhile, $w$ is a certain real analytic 1 -form 
on $S^{1} \times B$. Equation (5.8) and the added constraint that $d * \underline{\beta}=0$ form a linear, elliptic system of differential equations on $S^{1} \times B$, and standard elliptic regularity theorems (applied on $S^{1} \times B$ ) imply that both $\underline{\alpha}$ and $\underline{\beta}$ are real analytic differential forms on $S^{1} \times B$. This last result translates back to the annulus $A$ to yield the assertions of Lemma 5.3.

Step 6: This step completes the argument for the assertion that $D_{C}$ is Fredholm. In this regard, note first that $D_{C}$ has closed range; this is a direct consequence of Lemma 5.2 via a standard argument. With the range of $D_{C}$ closed, the cokernel of $D_{C}$ is isomorphic to the vector space of $\eta \in \mathcal{R}$ which are annihilated by a certain differential operator $D_{C}^{*}$. Here $D_{C}^{*} \eta=|\omega|^{-1} D_{C}^{\dagger}(|\omega| \eta)$ with $D_{C}^{\dagger}$ being the formal $L^{2}$ adjoint of $D_{C}$.

Now, $D_{C}^{*}$ is an elliptic operator and so $\eta$ is smooth. Moreover, on an annular end $A$, the operator $D_{C}^{*}$ can be written in terms of the coordinates $(\alpha, \beta)$ for the normal bundle $N$, and in this guise, it has the same schematic form as $D_{C}$ in (5.6). In particular, the analysis in the proof of Lemma 5.3 implies that any $s \in$ kernel $\left(D_{C}^{*}\right)$ with $\int_{C}|s|^{2} \omega<\infty$ lies in the $N \otimes T^{0,1} C$ version of the domain $\mathfrak{D}$. That is, $\int_{C}\left(|\nabla s|^{2}+|\omega|^{-2}|s|^{2}\right) \omega<\infty$. Then, the arguments for Lemmas 5.1-5.3 apply with no substantive modifications to show that $\int_{C}\left(|\nabla s|^{2}+|\omega|^{-2}|s|^{2}\right) \omega \leq \zeta \int_{K}|s|^{2} \omega$, where $K \subset C$ is a certain measurable set with compact closure, and where both $K$ and $\zeta$ are independent of the chosen $s \in \operatorname{kernel}\left(D_{C}^{*}\right)$. This last point then implies via standard arguments that the kernel of $D_{C}^{*}$ is finite dimensional.

Step 7: As before, assume here only that $C \in \mathcal{M}_{b}$ is immersed in $X-Z$. This step begins the construction of a map $f: \operatorname{kernal}\left(D_{C}\right) \rightarrow \operatorname{cokernel}\left(D_{C}\right)$ with an associated map $\mathcal{F}: f^{-1}(0) \rightarrow \mathcal{M}$. Both maps have a rough description in Subsection $5 \mathrm{~b}$, above. To obtain a rigorous definition, various auxiliary issues need to be covered. This step is devoted these preliminary tasks.

The first of these tasks is the introduction of the orthogonal projection $\Pi: \mathcal{R} \rightarrow \mathcal{R}$ which maps onto the image of $D_{C}$. In this regard, the metric to use is that which arises from the polarization of the norm $\|\cdot\|_{\mathcal{R}}$. This operator $\Pi$ is also the projection orthogonal to the kernel of the operator $D_{C}^{*}$. By definition, $D_{C}$ maps $\mathfrak{D}$ onto the image of $\Pi$ and it maps the orthogonal compliment of kernel $\left(D_{C}\right)$ isomorphically onto this image. (Define 'orthogonality' here using the metric which is obtained by polarizing the norm $\|\cdot\|_{\mathfrak{D}}$.) In particular, $D_{C}$ has a bounded inverse on $\Pi \mathcal{R}$, denoted by $D_{C}^{-1}$, which is defined to map $\Pi \mathcal{R}$ to the aforementioned orthogonal compliment in $\mathfrak{D}$ to kernel $\left(D_{C}\right)$.

The second task introduces a vector subspace $\mathfrak{D}^{\prime} \subset \mathfrak{D}$ which is mapped by $R_{1}$ in $(5.1)$ to $\mathcal{R}$. (Here and below, $R_{1}$ should be viewed as simply a map which sends a section of $N_{0}$ to one of $N \otimes T^{0,1} C$.) the definition of $\mathfrak{D}^{\prime}$ requires the introduction of a set $\mathcal{U}$ whose elements are subsets of $C$. Here, $D \in \mathcal{U}$ when $D \subset C$ is either a disk a radius $\varepsilon / 2$ or less, or else $D$ lies in an annular end $A$ and is the intersection of $A=S^{1} \times(0, \varepsilon)$ with a disk in $S^{1} \times[-\varepsilon, \varepsilon]$ and which has its center in $S^{1} \times[0, \varepsilon]$. Note that each $D \in \mathcal{U}$ has a well defined radius and the latter will be denoted by $r(D)$.

The definition of $\mathfrak{D}^{\prime}$ also requires the choice of a constant $\delta \in(0,1 / 1000)$. With $\delta$, fixed declare $s \in \mathfrak{D}^{\prime}$ when the following is finite:

$$
\|s\|_{\mathfrak{D}^{\prime}}^{2} \equiv\|s\|_{\mathfrak{D}}^{2}+\sup _{D \in \mathcal{U}} r(D)^{-\delta} \int_{D}\left(|\nabla s|^{2}+|\omega|^{-1}|s|^{2}\right)|\omega|^{-1} \omega
$$


According to Lemma 5.3, the kernel of $D_{C}$ lies in $\mathfrak{D}^{\prime}$. In any event, let $\mathfrak{D}^{\prime 1} \subset \mathfrak{D}^{\prime}$ denote the intersection of $\mathfrak{D}^{\prime}$ with the orthogonal compliment in $\mathfrak{D}$ to the kernel of $D_{C}$.

Here is the fundamental lemma concerning $\mathfrak{D}^{\prime}$ :

LEMMA 5.4. The vector space $\mathfrak{D}^{\prime}$ is a Banach space with the norm $\|\cdot\|_{\mathfrak{D}^{\prime}}$. Moreover, with $R_{1}$ from (5.1) viewed mapping sections of $N_{0}$ to those of $N \otimes T^{0,1} C$, then the latter maps $\mathfrak{D}^{\prime}$ into $\mathcal{R}$. In addition, $D_{C}^{-1} \Pi R_{1}$ defines a smooth map from a ball $\mathcal{B}^{\prime} \subset \mathfrak{D}^{\prime}$ about the origin to $\mathfrak{D}^{\prime} \perp$ whose derivative at $0 \in \mathfrak{D}^{\prime}$ vanishes.

This lemma is proved in Step 10.

Step 8: This step assumes Lemma 5.4 and then completes the construction of the maps $f$ and $\mathcal{F}$ in (5.4). As in the previous steps, the arguments here only require that $C \in \mathcal{M}_{b}$ be a immersed in $X-Z$.

To begin the arguments, introduce $\mathcal{T}: \mathfrak{B}^{\prime} \rightarrow \mathfrak{D}^{\perp}$ by the rule $\mathcal{T}(\cdot)=(\cdot)^{\perp}+$ $D_{C}^{-1} \Pi R_{1}(\cdot)$. Here, $s^{\perp}$ is the projection of $s$ onto $\mathfrak{D}^{\prime} \perp$. Since $D_{C}^{-1} \Pi R_{1}(\cdot)$ has vanishing derivative at the origin, the implicit function theorem can be applied to obtain a ball $B \subset \operatorname{kernel}\left(D_{C}\right)$ containing 0 together with a smooth map $s_{1}: B \rightarrow \mathfrak{D}^{\prime} \perp$ with the property that $\mathcal{T}\left(s_{0}+s_{1}\left(s_{0}\right)\right)=0$ and $\left\|s_{1}\right\|_{\mathfrak{D}^{\prime}} \leq \zeta\left\|s_{0}\right\|_{\mathfrak{D}^{2}}^{2}$. Here, $\zeta$ is independent of $s_{0}$. With $s_{1}$ understood, define the map $f: B \rightarrow \operatorname{cokernel}\left(D_{C}\right)$ by setting $f\left(s_{0}\right) \equiv(1-\Pi) R_{1}\left(s_{0}+s_{1}\left(s_{0}\right)\right)$. The map $\mathcal{F}$ of $(5.4)$ is defined on the whole of $B$ by the exponential map; so by construction, it embeds $f^{-1}(0)$ into a neighborhood in $\mathcal{M}$ of the given pseudoholomorphic submanifold $C$.

Step 9: This step completes the proof of Proposition 2.6 under the assumption that $C$ is immersed. In particular, with the map $\mathcal{F}$ of (5.4) understood, the time has come to consider the image of $\mathcal{F}$. In particular, Proposition 2.6 assets that when $C$ lies in $\mathcal{M}_{b, k}$, then $\mathcal{F}$ maps $B$ onto a neighborhood of $C$ in $\mathcal{M}_{b, k}-\mathcal{M}_{b, k+1}$. And, this conclusion follows in a straightforward manner from the implicit function theorem construction of $\mathcal{F}$ provided that each $C^{\prime}$ from some neighborhood in $\mathcal{M}_{b, k}$ of $C$ is the image (via the exponential map) of a section of $C$ 's normal bundle. And, the latter conclusion follows directly from Lemma 4.3, Lemma 3.7 and (4.3).

\section{Step 10: This step contains the}

Proof of Lemma 5.4. To prove that $\mathfrak{D}^{\prime}$ is a Banach space with the norm whose square appears on the right side of (5.9), it is enough to prove that Cauchy sequences in this norm have limits in $\mathfrak{D}^{\prime}$. This task is straightforward and left to the reader. The assertion that $R_{1}$ maps a non empty ball $\mathcal{B}^{\prime} \subset \mathfrak{D}^{\prime}$ to $\mathcal{R}$ follows from the assertion that the $L^{\infty}$ norm is bounded on the unit ball in $\mathfrak{D}^{\prime}$. Then, the latter assertion follows from Theorem 3.5.2 in [Mo] after a minor modification of the latter to account for the fact that the metric closure of $C$ has a boundary. (See, e.g. the proof of Lemma 8.3 in [T2].) In fact, Theorem 3.5.2 implies that there exists $\zeta>0$ such that the Holder norm with exponent $\zeta$ is bounded on the unit ball in $\mathfrak{D}^{\prime}$.

The proof that $D_{C}^{-1}$ maps $\Pi R_{1}(\cdot)$ into $\mathfrak{D}^{\prime} \perp$ is obtained in two parts. To begin, introduce the shorthand $q \equiv D_{C}^{-1} \Pi R_{1}(s)$ for $s \in \mathcal{B}^{\prime}$. There are two parts to the argument that $q \in \mathfrak{D}^{\prime}$. The first finds a constant $\zeta>0$ which is independent of $s$ and 
has the following significance: When $D \subset \mathcal{U}$ has center in $C-A$, then

$$
\int_{D}\left(|\nabla q|^{2}+|\omega|^{-1}|q|^{2}\right)|\omega|^{-1} \omega \leq \zeta r(D)^{\delta}\|s\|_{\mathfrak{D}^{\prime}}^{2} .
$$

Indeed, the existence of such $\zeta$ can be deduced directly from Theorem 5.4.1 in [Mo] using the equation $D_{C} q=\Pi R_{1}$.

With (5.10) understood, it remains only to obtain the inequality in (5.10) for those $D \subset \mathcal{U}$ which lie in $A$. To derive this version of (5.10), first write $q$ in terms of a pair $(\alpha, \beta)$ so that $D_{C} q$ is given by (5.6). Then, introduce $(\underline{\alpha}, \beta)$ as in (5.8). This translation rewrites the equation $D_{C} q=\Pi R_{1}$ as an equation on $S^{1} \times B$ which has the schematic form

$$
d \underline{\alpha}+* d \underline{\beta}+*(w \wedge \underline{\beta})=\underline{\pi}, .
$$

with the auxiliary condition that $d * \underline{\beta}=0$. Here $\underline{\pi}$ is an square integrable real line bundle valued 1-form on $S^{1} \times B$ (using the product metric). Moreover, if $\underline{D} \subset S^{1} \times B$ is a ball, and if $r$ denotes the radius of $\underline{D}$, then $\int_{\underline{D}}|\underline{\pi}|^{2} \mathrm{dvol} \leq \zeta r^{\delta}\|s\|_{\mathfrak{D}}$. It then follows from this last fact using Theorem 5.3.7 in [Mo] that

$$
\int_{\underline{D}}\left(|\nabla \underline{\alpha}|^{2}+|\nabla \underline{\beta}|^{2}\right) \mathrm{dvol} \leq \zeta r^{\delta}\|s\|_{\mathfrak{D}^{\prime}} .
$$

After some straightfoward manipulations, this last equation implies that

$$
\int_{D}\left(|d \alpha|^{2}+u^{-6}\left|d\left(u^{3} \beta\right)\right|^{2}\right) u^{3} d \tau d u \leq \zeta r(D)^{\delta}\|s\|_{\mathfrak{D}^{\prime}}^{2}
$$

for all $D \in \mathcal{U}$ which lie in $A$. And, it is a straightfoward matter (which is left to the reader) to derive the $D \subset A$ version of (5.10) from this last equation.

d) The Proof of Proposition 2.6 in the general case. The argument for Proposition 2.6 are organized into six steps, with the first providing an overview of sorts.

Step 1: In the case where $Z=\phi$, the discussion is fairly standard (see, e.g. [MS] or Section 7 of [T5].) The strategy followed here mimics that used in the $Z=\phi$ case. The basic point is that deformation via sections of the normal bundle can not be used when $C$ is not immersed, and the replacement considers not a deformation of $C$ but a deformation of the map $\sigma$ from the smooth model $C_{0}$. Using an exponential map on $X$, suitably small deformations of the latter which are pseudoholomorphic correspond to sections over $C_{0}$ of $\sigma^{*} T X$ which obey a nonlinear equation whose symbol is the same as that of $\bar{\partial}$. The outline in Section $5 \mathrm{~B}$ is then applied in this new context to give the desired result. In particular, since the non-immersed points of $C$ are away from $Z$, the required analysis marries the discussion in the previous subsections (which is still valid near $Z$ ) to the standard analysis as found in [MS] which is valid away from $Z$. The steps along the way are straightforward for the most part, and so the discussion will be brief in as much as it will touch only on the points where the path to follow is not fairly obvious. In particular, these points all involve the operator which replaces the operator $D_{C}$ in the previous subsections. 
Step 2: As just noted, the complications here arise because $C_{0}$ is not compact. However, $C_{0}$ does have a natural compacification, $\underline{C}_{0}$ to which $\sigma$ extends and maps the boundary of $Z$ as a local covering. Moreover, as noted previously in Section $2 \mathrm{~b}, \sigma^{*} T X$ extends over the boundary as a complex vector bundle. This means that the deformation problem that one studies in the case of compact $C_{0}$ can be formally set up here. The only novelty is that of setting boundary conditions for sections of $\sigma^{*} T X$ on $\partial \underline{C}_{0}$.

In order to specify the appropriate boundary conditions, it is crucial to realize that a section $s$ of $\sigma^{*} T^{0,1} C_{0}$ can be written near $\partial C_{0}$ in terms of a section, $s_{0}$, of $T^{0,1} C_{0}$ and a section $s_{1}$ of $\sigma^{*} N$. In this regard, note that $T^{0,1} C_{0}$ has a canonical basis along $\partial C_{0}$, that being the vector field $\partial_{t}-i \partial_{u}$. This basis identifies a section of $T^{0,1} C_{0}$ over $\partial C_{0}$ with a complex valued function on $\partial C_{0}$. And, with this identification understood, restrict attention here to sections $s$ of $\sigma^{*} T^{0,1} X$ for which the corresponding $s_{0}$ is real along the $\partial C_{0}$. Such a section defines a first order deformation of $C_{0}$ which keeps $\partial C_{0}$ in $Z$.

Meanwhile, an additional restriction on the section $s$ of $\sigma^{*} T^{0,1} X$ arises from consideration of the $\sigma^{*} N$ part of $s$ near $\partial C_{0}$. In particular, consider only sections $s$ where the corresponding $\sigma^{*} N$ component, $s_{1}$, is such that $\left(\left|\nabla s_{1}\right|^{2}+u^{-2}\left|s_{1}\right|^{2}\right) \sigma^{*} \omega$ is integrable near $\partial C_{0}$. (The reader should note that this is the same condition which was imposed in Section 5a.)

Required now is a Hilbert space completion of the just described space of sections. Here is the appropriate one: Complete the space of smooth sections of $\sigma^{*} T^{0,1} X$ whose $s_{1}$ component is real on $\partial C_{0}$ using the norm whose square sends a section $s$ to

$$
\int_{C_{0}}\left(\left|\nabla\left((1-\chi) s+\chi s_{0}\right)\right|^{2}+\left|\left((1-\chi) s+\chi s_{0}\right)\right|^{2} d A+\int_{C_{0}}\left(\left|\nabla\left(\chi s_{1}\right)\right|^{2}+u^{-2}\left|\chi s_{1}\right|^{2}\right) \sigma^{*} \omega .\right.
$$

Here $\chi$ is the pull-back via $\sigma$ to $\underline{C}_{0}$ of a smooth function which is one on some small radius tubular neighborhood of each component of $Z$ and zero on the compliment of a slightly larger tubular neighborhood. Meanwhile, $d A$ is the induced area form. Call the resulting Hilbert space $\mathfrak{D}$.

Also required is the Hilbert space $\mathcal{R}$ which is obtained by completing the space of sections of $\sigma^{*} T X$ using the norm whose square sends $s$ to

$$
\int_{C_{0}}\left|\left((1-\chi) s+\chi s_{0}\right)\right|^{2} d A+\int_{C_{0}}\left|\chi s_{1}\right|^{2} \sigma^{*} \omega
$$

(No additional boundary conditions are imposed on the $s_{1}$ part of sections from $\mathcal{R}$.)

Step 3: The condition that a small section $s$ of $\sigma^{*} T^{0,1} X$ defines a pseudoholomorphic deformation of $C$ amounts to a nonlinear equation for $s$ whose formal linearization defines an operator, $D_{C}^{\prime}$, with the schematic form

$$
D_{C}^{\prime}=\bar{\partial}+R_{0}(\cdot) .
$$

Here, $R_{0}(\cdot)$ is a certain $\mathbb{R}$-linear endomorphism from $\sigma^{*} T X$ to $\sigma^{*} T X \otimes T^{0,1} C_{0}$.

Note that $D_{C}^{\prime}$ extends as a bounded operator from $\mathfrak{D}$ to $\mathcal{R}$. Moreover,

LEMMA 5.5. The operator $D_{C}^{\prime}$ from (5.21) induces a Fredholm operator from $\mathfrak{D}$ to $\mathcal{R}$.

The remainder of this step is occupied with the 
Proof of Lemma 5.5. The proof requires a well known result about boundary condition specifications for the $\bar{\partial}$ operator. A digression which starts here states and proves the required result. Here is the statement:

Lemma 5.6. Let $C^{\prime}$ be a compact, Riemann surface with boundary. Let $E \rightarrow C^{\prime}$ be a hermitian, holomorphic line bundle and suppose that $s_{0}$ is a unit length section of $E$ along $\partial C^{\prime}$. Use $C^{\infty}\left(E, s_{0}\right)$ to denote the space of sections $s$ of $E$ which are real multiplies of $s_{0}$ along $\partial C^{\prime}$. Meanwhile, suppose that $R_{0}$ is a smooth section of the bundle of $\mathbb{R}$ linear homomorphisms from $E$ to $E \otimes T^{0,1} C^{\prime}$ and then let $D$ denote the $\mathbb{R}$-linear operator $\bar{\partial}+R_{0}: C^{\infty}\left(E, s_{0}\right) \rightarrow C^{\infty}\left(E \otimes T^{0,1} C^{\prime}\right)$. Then $D$ extends as a Fredholm operator from the $L_{1}^{2}$ completion of $C^{\infty}\left(E, s_{0}\right)$ to the $L^{2}$ completion of $C^{\infty}\left(E \otimes T^{0,1} C^{\prime}\right)$.

Proof of Lemma 5.6. Because $C^{\prime}$ is compact and $R_{0}$ is zero'th order, it is sufficient to consider the case where $R_{0}=0$. In this case, integration by parts finds that

$$
\int_{C^{\prime}}|\bar{\partial} s|^{2} \geq \int_{C^{\prime}}\left(|\nabla s|^{2}-\zeta|s|^{2}\right)-\zeta \int_{\partial C^{\prime}}|s|^{2},
$$

where $\zeta$ is a constant. In this regard, note that there would ordinarily be a term in (5.17) which involves the integration over $\partial C^{\prime}$ of a bilinear in $s$ and $\nabla s$, but the latter vanishes under the assumption that $s$ is a real multiple of $s_{0}$ on $\partial C^{\prime}$. In the usual way, (5.17) implies that $D$ has closed range and finite dimensional kernel on the $L_{1}^{2}$ completion of $C^{\infty}\left(E, s_{0}\right)$. (Remember that the map from $L_{1}^{2}$ functions to $L^{2}$ functions on the boundary is compact [Ad].)

Since the range of $D$ is closed, its cokernel can be identified with the kernel of the formal $L^{2}$ adjoint of $D$ acting on sections of $C^{\infty}\left(E \otimes T^{0,1} C^{\prime}\right)$ which are imaginary multiples of a certain fiducial section $E \otimes T^{0,1} C^{\prime}$ along $\partial C^{\prime}$. In this regard, note that $T^{0,1} C^{\prime}$ has a canonical section along $\partial C^{\prime}$, namely the projection of the tangent vector to the boundary, while $E$ has the given section $s_{0}$. In any event, an analog of (5.17) holds for this adjoint of $D$ and this means that the cokernel of $D$ is also finite dimensional.

With the digression for Lemma 5.6 now over, the proof of Lemma 5.5 is obtained by coupling the arguments in Steps 1-6 of the proof of Proposition 2.6 in Section 5c with those for Lemma 5.6. The details are completely straightforward and left to the reader. Alternately, excision arguments can be invoked since $D_{C}^{\prime}$ splits naturally enough near $\partial C_{0}$ into a direct sum of operators for which the Fredholm story has already been told.

Step 4: With the operator $D_{C}^{\prime}$ understood, the remainder of the argument for Proposition 2.6 proceeds as in Steps 7-9 of Section 5c and as outlined in Section 5b. The details here are left to the reader save for two comments about the operator to use to play the role of $D_{C}$ in Sections $5 \mathrm{~b}$, c. The point is that one should not use $D_{C}^{\prime}$, but use instead an operator which is obtained from $D_{C}^{\prime}$ by restricting the latter to the compliment of a certain finite dimensional subspace of the domain $\mathfrak{D}$ and then projecting orthogonal to a certain finite dimensional subspace of the range $\mathcal{R}$. (Both projections are required even when $C_{0}$ is compact.)

Consider first the range projection: Its purpose is to allow the complex structure on $C_{0}$ to vary when $\sigma$ is deformed. In any event, this finite dimensional subspace is the image via push-forward by the differential of $\sigma$ of the cokernel in $T_{1,0} C_{0} \otimes T^{0,1} C_{0}$ of the image of $\bar{\partial}$. Here $\bar{\partial}$ is operating on the $L_{1}^{2}$ closure of the space of sections $s_{0}$ of 
the holomorphic tangent bundle of $\underline{C}_{0}$ which are real on $\partial \underline{C}_{0}$. Meanwhile, the range space for $\bar{\partial}$ is the $L^{2}$ closure of the space of sections of $T_{0,1} C_{0} \otimes T^{0,1} C_{0}$.

The trimming of the domain $\mathfrak{D}$ compensates for the possible presence of nontrivial diffeomorphisms of $\underline{C}_{0}$ which are close to the identity, preserve $\partial \underline{C}_{0}$ and are holomorphic on $C_{0}$. (Such diffeomorphisms exist only on spherical, cylindrical, toroidal and disk components of $C_{0}$.) In particular, the finite dimensional subspace of $\mathfrak{D}$ which is involved here is also pushed forward by the differential of $\sigma$. Indeed, it is the push-forward of the space of sections of the holomorphic tangent bundle of $\underline{C}_{0}$ which are in the kernel of $\bar{\partial}$ and which are real on $\partial \underline{C}_{0}$.

e) The proof of Proposition 2.8. Observe first that it is sufficient to consider the validity in (2.5) solely for the case where $C$ is immersed. Here is why: The subvariety $C$ determines its smooth model curve, $C_{0}$ with the pseudoholomorphic map $\sigma: C_{0} \rightarrow X-Z$. If $\sigma$ is not an immersion, there is, in any event, a perturbation of $\sigma$ which results in a map $\sigma^{\prime}$ which immerses $C_{0}$ with solely positive double points, agrees with $\sigma$ near $\partial \underline{C}_{0}$, and pulls $\omega$ back as a strictly positive form. Moreover, the map $\sigma^{\prime}$ can be as close to $\sigma$ as desired in any given $C^{k}$ topology. Since $C^{\prime}=$ image $\left(\sigma^{\prime}\right)$ is immersed, symplectic and has only positive double points, the map $\sigma^{\prime}$ is pseudoholomorphic for certain perturbed complex structures on $C_{0}$ and $X-Z$. In particular, these almost complex structures can be taken close to the originals (and that on $X-Z$ equal to the original near $Z$ ) by taking $\sigma^{\prime}$ to be close to $\sigma$. The point here is that the right hand side of (2.5) can be computed using these new complex structures as well as the old. Meanwhile, the new version of the operator $D_{C}$ will be a perturbation of the old (as long as $\sigma^{\prime}$ is close to $\sigma$ ) and so the left hand side of (2.5) can also be computed using the new data instead of the old.

With the preceding understood, assume now that $C$ is immersed. In this case, the right hand side of $(2.5)$ is $\chi\left(C_{0}\right)+2 d_{C}+N^{\prime}(C)$, where $d_{C}$ is the degree of the normal bundle to the immersion as defined using sections whose behavior near $\mathrm{Z}$ are described in Step 4 of Section 2b. (This is because $\sigma^{*} T X$ splits as $T C_{0} \otimes N$ when $C$ is immersed.) Thus, the goal here is a proof of the assertion that the index of $D_{C}$ is equal to $\chi\left(C_{0}\right)+2 d_{C}+N^{\prime}(C)$. The following six steps achieve this goal.

Step 1: Consider first the case where $C$ has a compact component $C^{\prime}$. Here, the index of $D_{C}$ is equal to the real index of the $\bar{\partial}$ operator for $C_{0}^{\prime}$ acting on sections of the normal bundle to $C^{\prime}$. the Riemann-Roch theorem finds the latter equal to $\chi\left(C_{0}^{\prime}\right)+2 d_{C^{\prime}}$.

Step 2: Consider here a component $C$ which is non-compact. To save notation, this component will be denoted also by $C$. The strategy here is to compare $D_{C}$ with an appropriate $\bar{\partial}$ operator on a compact Riemann surface which is obtained from $\underline{C}_{0}$ by capping each end of the latter with a disk.

The discussion starts with the observation that the inequality in Lemma 5.2 allows $D_{C}$ to be continuously deformed through first order, Fredholm differential operators to obtain an operator, $D_{C}^{\prime}$ with the following properties: There exists $\varepsilon>0$ and define with this $\varepsilon$, near each component of $\mathrm{Z}$, the usual annulus $A=S^{1} \times(0, \varepsilon] \subset C$. Then, require that $D_{C}^{\prime}$ is $\mathbb{C}$-linear on the compliment of the union of these annuli. Meanwhile, on $S^{1} \times(0, \varepsilon / 2] \subset A$, the operator $D_{C}^{\prime}$, when written using the variables $(\alpha, \beta)$, is the restriction from $S^{1} \times(0, \infty)$ of an operator $D_{0}$ which sends $(\alpha, \beta)$ to

- $\alpha_{\tau}-\beta_{u}-3 \chi(u) u^{-1} \beta$, 
- $\alpha_{u}+\beta_{\tau}$.

Here, $\chi$ is a favorite non-increasing, smooth function on $[0, \infty)$ which is identically 1 near 0 and identically zero on the half line $[\varepsilon / 2, \infty)$.

Step 3: The advantage of using $D_{C}^{\prime}$ arises because the kernel of the operator $D_{0}$ in (5.19) can be written down in a fairly explicit manner by separating variables. In particular, a linearly independent generating set $\mathcal{Q}=\cup_{n} \mathcal{Q}_{n}$ of elements in the kernel of $D_{0}$ is described below. Here, $n$ ranges over the nonnegative integers when the $z$-axis line bundle pulls back to the annulus $A$ as an oriented bundle; otherwise, $n$ ranges over the positive element in $\mathbb{Z}+1 / 2$. This set $\mathcal{Q}$ generates the kernel of $D_{0}$ in the following sense: Each element in the kernel the restriction of $D^{\prime}{ }_{0}$ to any annulus $S^{1} \times(a, b)$ for $0 \leq a<b \leq \infty$ is a suitable linear combination of elements of the set $\mathcal{Q}$.

The set $\mathcal{Q}_{0}$ consists of the pair of solutions,

- $(1,0)$.

- $\left(0, k(u) \equiv \exp \left(3 \int_{s \geq u} \chi(s) s^{-1} d s\right)\right)$.

Meanwhile, for each non-zero $n$, the corresponding $\mathcal{Q}_{n}$ consists of four solutions having the form

- $\left(a_{ \pm n}(u) \cos \left(E_{n} \tau\right), b_{ \pm n}(u) \sin \left(E_{n} \tau\right)\right)$.

- $\left(-a_{ \pm n}(u) \sin \left(E_{n} \tau\right), b_{ \pm n}(u) \cos \left(E_{n} \tau\right)\right)$.

where $E_{n} \equiv 2 \pi n / q$. In this last equation $\left(a_{+n}, b_{+n}\right)$ and $\left(a_{-n}, b_{-n}\right)$ are two pair of functions of $u$ which depend on the label $n$. In this regard, for small values of $u$, the leading order behavior of $\left(a_{+n}, b_{+n}\right)$ and $\left(a_{-n}, b_{-n}\right)$ is given by

$$
\begin{gathered}
a_{+n}=1+8^{-1} E_{n}^{2} u^{2}+\cdots, \\
a_{-n}=2^{-1} E_{n}^{-2} u^{-2}+4^{-1} \ln \left(E_{n} u\right) \cdots, \\
b_{-n}=E_{n}^{-3} u^{-3}-4^{-1} E_{n}^{-1} u^{-1}+\cdots
\end{gathered}
$$

In this last equation, the notation '...' indicates a function of $u$ which is a higher order in $u$ as those indicated. In particular, the $a_{+n}$ version of this function vanishes as $u^{4}$ and the $b_{+n}$ as $u^{3}$.

On the other hand, $\left(a_{+n}, b_{+n}\right)$ are $\mathcal{O}\left(e^{E_{n} u}\right)$ for large values of $u$, while $\left(a_{-n}, b_{-n}\right)$ are characterized by the condition that this pair is $\mathcal{O}\left(e^{-E_{n} u}\right)$ as $u$ gets large. In this regard, note that the maximum principle implies that subspace in kernel $\left(D_{0}\right)$ of bounded elements is precisely the $\mathbb{R}$-linear span of the solution in the top line of (5.20). Moreover, there are no $\psi \in \operatorname{kernel}\left(D_{0}\right)$ with $\int|\psi|^{2} k(u)^{-1} d t d u<\infty$. (Remember that $k(u)$ is defined in the second line of (5.20). In particular, as $u$ tends to zero, $k(u) \sim u^{-3}$, while $k(u) \sim$ constant as $u$ gets large.)

(Note: If the length of the component of $\mathrm{Z}$ here is some $l_{Z} \neq 2 \pi$, then replace $q$ in the expression for $E_{n}$ by $q l_{Z} / 2 \pi$.)

Step 4: As the index of $D_{C}$ is the same as that of $D_{C}^{\prime}$, it is sufficient to compute the index of the latter. With this understood, the simplest case to consider is the case where the $z$-axis line bundle pulls back to each annulus A as an oriented bundle. This assumption will be made in this step and in Step 5 .

To begin the discussion, note that any element in the kernel of $D_{C}^{\prime}$ must restrict to $(0, \varepsilon / 2)$ in the kernel of $D_{0}$. As such an element has bounded $(\alpha, \beta)$ as $u \rightarrow 0$, it 
must lie in the linear span of the set $\mathcal{Q}_{+} \subset \mathcal{Q}$ whose members consist of the solution in the top line of (5.20) and those elements in (5.21) with the + subscript.

Another way to phrase the preceding conclusion is that the complex valued function $\alpha+i \beta$ must restrict to the circle $u=\varepsilon / 2$ as an $\mathbb{R}$-linear combination of the constant function 1 and the complex exponentials $\left\{\lambda e^{i E_{n} \tau}: \lambda \in \mathbb{C}\right\}_{n>0}$. In particular, this last observation implies the following : If each end of $C$ is capped off with a disk, the given element in the kernel of $D_{C}^{\prime}$ extends as a holomorphic function over each such disk.

The preceding observation can be further rephrased as follows: Let $N(C)$ denote the number of ends of $C$. Then, the kernel of $D_{C}^{\prime}$ on $C$ is isomorphic to the zero set of $N(C)$ linear equations on the kernel of a certain $\bar{\partial}$-operator on the compact, complex curve $C_{*}$ which is obtained from $C$ by attaching disks to each boundary component. In particular, the $\bar{\partial}$ operator in question here is coupled to the complex line bundle over $C_{*}$ whose degree is the self intersection number of $C$ as defined by pushing $C$ off itself in the $+\mathrm{z}$ direction near each component of $\mathrm{Z}$.

A similar analysis for the cokernel of $D_{C}^{\prime}$ finds that the cokernel of the aforementioned twisted $\bar{\partial}$ operator of $C_{*}$ is the zero set of $N(C) \mathbb{R}$-linear equations on the cokernel of $D_{C}^{\prime}$. The point here is the following: If $\psi$ is an element in the cokernel of the twisted $\bar{\partial}$ operator, then $\psi^{\prime}$ s restriction to the $u=\varepsilon / 2$ circle, when written as $\alpha+i \beta$, must have zero constant term in its Fourier expansion. However, the analogous constant term for an elements in the cokernel of $D_{C}^{\prime}$ is constrained only to lie in the real, dimension 1 subspace of $\mathbb{C}$ which is defined by the restriction of the solution in the first line of (5.20).

Step 5: The preceding identifications imply that index $\left(D_{C}^{\prime}\right)=\chi\left(C_{*}\right)+2 d_{C}-$ $N(C)$, where $\chi\left(C_{*}\right)$ now denotes the Euler characteristic of $C_{*}$. Note that this number is $\chi(C)+2 d_{C}$, as required.

The conclusion that index $\left(D_{C}^{\prime}\right)=\chi\left(C_{*}\right)+2 d_{C}-N(C)$ is easiest to see when the real index of the $\bar{\partial}$ operator on $C_{*}$ is at least $2 N(C)$ and when the index of $D_{C}^{\prime}$ is positive. In this case, a generic, $\mathbb{C}$-linear perturbation of $D_{C}^{\prime}$ with support on A's compliment will have three effects: First, the perturbed operator will again determine a corresponding $\bar{\partial}$ operator on $C_{*}$. Second, the resulting perturbation of $D_{C}^{\prime}$ will have trivial cokernel, and thus so will the corresponding $\bar{\partial}$ operator in $C_{*}$. Third, a certain $\mathbb{C}$-linear map from the kernel of this $\bar{\partial}$ operator to $\otimes_{N(C)} \mathbb{C}$ will be surjective. Here, the coordinates of this map correspond to a labeling of the ends of $C$, and the i'th coordinate is evaluation at the center point of the added disk which corresponds to the i'th end.

Given that the aforementioned map is surjective, it follows immediately that the $N(C)$ real equations which cut the kernel of the perturbed $D_{C}^{\prime}$ out of the kernel of the corresponding $\bar{\partial}$ operator on $C_{*}$ are linearly independent. This last point implies that the index of $D_{C}^{\prime}$ is $N(C)$ less than that of the corresponding $\bar{\partial}$ operator on $C_{*}$. As the index of the latter is $\chi\left(C_{*}\right)+2 d_{C}$, the result follows.

The argument for the general case can be made by a similar comparison of the kernels and cokernels of $D_{C}^{\prime}$ and the $\bar{\partial}$ on $C_{*}$. Alternately, the general case can be reduced to the preceding one using the excision property of the index.

Step 6: In this step, assume that the $z$-axis line bundle restricts from a tubular neighborhood of $Z$ as an orientable line bundle to some number $N(C)$ of ends of $C$, while its restriction to some number $N^{\prime}(C)$ of ends is not orientable. Construct as 
before the compact, complex curve $C_{*}$ by capping off the ends of $C$ with disks. Once again, the kernel of the operator $D_{C}^{\prime}$ can be identified as the solution space of some $N(C) \mathbb{R}$-linear equations on the kernel of the $\bar{\partial}$ operator on $C_{*}$ coupled to a certain complex line bundle over $C_{*}$. In fact, just as before, such end of $C$ which approaches a component of $Z$ with oriented $z$-axis accounts for precisely one of these real equations. The other ends of $Z$ do not contribute to these linear equations.

Meanwhile, the complex line bundle in question has a degree which is defined using sections near $Z$ which behave as follows: The section pushes off towards the $+z$-axis along each component of $Z$ with oriented $z$-axis. To define the section along a component $Z^{\prime} \subset Z$ with unoriented $z$-axis, it is sufficient to consider the section along the circle where $u=\varepsilon / 2$. Here, choose any nowhere vanishing section $s=(\alpha, \beta)$ of the normal bundle to $C$ along this circle with the following properties:

- $\left.(\alpha, \beta)\right|_{\tau=0}=(1,0)$.

- $\left.(\alpha, \beta)\right|_{\tau=2 \pi}=(-1,0)$.

- $\left.\nabla_{\tau}(\alpha, \beta)\right|_{\tau=0}=(0,-1)$.

- $(\alpha, \beta) \neq( \pm 1,0)$ except where $\tau=0$ and $\tau=2 \pi$.

The reader can check that the section near $\mathrm{Z}$ just described is homotopic through non-vanishing sections of $N$ to that which is given in Step 4 of Section $2 b$. Thus, the degree in question for the complex line bundle is $d_{C}$.

With the preceding understood, the arguments in the previous two steps can be repeated essentially verbatim to conclude that index $\left(D_{C}^{\prime}\right)=\chi\left(C_{*}\right)+2 d_{c}-N(C)=$ $\chi(C)+2 d_{c}+N^{\prime}(C)$.

6. The appearance of $\boldsymbol{q}_{ \pm}$. The purpose of this section is to provide proofs of Proposition 2.11 and 2.12 .

a) The proof of Proposition 2.11. The proof of this proposition starts with a digression to state a lemma about perturbations of the given $J$ in a small ball. In the statement of the lemma, a symplectic subvariety of a ball is said to have finite energy when the integral of the symplectic form over the subvariety is finite.

Lemma 6.1. Let $B_{1}$ and $B_{4}$ denote the standard balls of radius 1 and 4 in $\mathbb{R}^{4}$ with center at the origin, and let $\omega$ denote the standard symplectic form on $\mathbb{R}^{4}$. Let $J$ be an $\omega$-compatible, almost complex structure on $B_{4}$ and let $C \subset B_{4}$ be a properly embedded finite energy, $J$-pseudoholomorphic subvariety which contains the origin. Now, let $\mathcal{J}$ denote the space of smooth, $\omega$-compatible, almost complex structures on $B_{4}$ which agree with $J$ on a neighborhood of $B_{4}-B_{1}$ in $B_{4}$. And, let $\mathcal{J}_{0} \subset \mathcal{J}$ denote the subset of complex structures which admit a finite energy, pseudoholomorphic subvariety which contains the intersection of $C$ with $B_{4}-B_{1}$. Then $\mathcal{J}-\mathcal{J}_{0}$ is a Baire set, being a countable intersection of open and dense subsets of $\mathcal{J}$.

Given the preceding lemma, here is how to prove Proposition 2.11: Suppose that $(g, \omega)$ does not lie in $\mathcal{G}^{\prime}$. Then either $q_{+}, q_{-}$is non-zero, or both are non-zero. The argument below assumes that $q_{+}=1$ and $q_{-}=0$. Except for some sign changes, the same argument works for the case $q_{-}=0$ and $q_{+}=1$. The argument for the case where both $q_{ \pm}=1$ also requires only cosmetic changes to the argument that follows. In any event, the argument for these last two cases is omitted.

To begin, fix some small $\varepsilon>0$ and let $B$ be a ball of radius $20 \varepsilon$ with center on $Z$ and such that the pair $(g, \omega)$ is described by (1.1) in $B$. Now, fix a ball $B_{4} \subset B$ of radius $4 \varepsilon$ whose center lies on the positive $z$ portion of the $t-z$ plane at distance at $8 \varepsilon$ 
from where $z=0$. Let $B_{1} \subset B_{4}$ denote the concentric ball of radius $\varepsilon$.

Now, let $J$ denote the almost complex structure $\sqrt{ } 2 g^{-1} \omega /|\omega|$ and consider an $\omega$-compatible almost complex structure, $J^{\prime}$, which agrees with $J$ except in a compact subset of the interior of $B_{1}$. Together, $J^{\prime}$ and $\omega$ define a pair $\left(g^{\prime}, \omega\right) \in \mathcal{G}$.

If this pair does not lie in $\mathcal{G}^{\prime}$ and is sufficiently close to $(g, \omega)$, then there will exist a $J^{\prime}$-pseudoholomorpic subvariety $C^{\prime} \subset X-Z$ which also contains some non-empty neighborhood of $z=0$ in the $z>0$ portion of the $t-z$ plane which lies in the ball $B$. (Of course, $C^{\prime}$ might contain some part of the $z<0$ portion of the $t$ - $z$ plane, but if this were true for all $J^{\prime}$ near to $J$, then by Proposition 3.8 in [T2], the invariant $q_{-}$ would equal one for $C$.)

There is a version of the unique continuation principle which is valid for pseudoholomorphic submanifolds. The latter implies, in part, the following: Let $U$ be a connected, open subset of the intersection of the original ball $B$ with the $z>0$ portion of the $t-z$ plane. If there are points with sufficiently small values of $z$ in $U$, then $U \subset C^{\prime}$. (This unique continuation principle follows from the fact that pseudoholomorpic subvarieties are manifolds on the compliment of a countable set of points. See, e.g. [PW], [Ye], [Pan], [MS].) With this last point understood, it follows that $C^{\prime}$ contains the annulus $A$ in the $t-z$ plane given by intersection of the latter with $B_{4}-B_{1}$.

On the other hand, according to Lemma 6.1, there is a Baire subset of $\omega$ compatible perturbations of $J$ such that any such $J^{\prime}$ differs from $J$ only in $B_{1}$ and admits no pseudoholomorphic subvariety of $B_{4}$ which contains the annulus $A$.

Proof of Lemma 6.1. First of all, by restricting to a smaller ball inside $B_{1}$, and then rescaling the metric, one can restrict to the case where the given $J$ is a small perturbation of the standard almost complex structure $J_{0}$, and where $C$ is a properly embedded disk in $B_{4}$ which is a small perturbation of the disk about the origin in the $t$ - $z$ plane. (Here, $\mathbb{R}^{4}$ is given coordinates $(t, z, x, y)$ and $\omega=d t \wedge d z+$ $d x \wedge d y$, while $J_{0}=\partial_{t} d z-\partial_{z} d t+\partial_{x} d y-\partial_{y} d x$.) For $E>0$, let $\mathcal{J}^{E} \subset \mathcal{J}$ denote the subset for which the following statement is true: No pseudoholomorphic subvariety with energy $\int \omega \leq E$ contains the intersection of $C$ with $B_{4}-B_{1}$. The proposition follows with a demonstration that $\mathcal{J}^{E}$ is open and dense for all positive numbers $E$.

The proof that $\mathcal{J}^{E}$ is open argues that the compliment, $\mathcal{J}-\mathcal{J}^{E}$ is closed. For this purpose, consider $J^{\prime} \in \mathcal{J}$ and a sequence $\left\{J_{i}\right\}$ in $\mathcal{J}-\mathcal{J}^{E}$ which converges to $J^{\prime}$. By assumption, for each $i$, there is a finite energy, $J_{i}$-pseudoholomorphic subvariety $C_{i} \subset B_{4}$ which contains the intersection, $A$, of $C$ with $B_{4}-B_{1}$ and which has energy no greater than $E$. With this understood, appeal to Proposition 3.8 in [T2] to deduce that the sequence $\left\{C_{i}\right\}$ has a subsequence which converges geometrically to a limit that includes a pseudoholomorphic subvariety for $J^{\prime}$ which also contains $A$ and which has energy no greater than $E$. Thus, $J^{\prime} \in \mathcal{J}-\mathcal{J}^{E}$.

To see that $\mathcal{J}^{E}$ is dense in $\mathcal{J}$, argue as follows: Take $J^{\prime} \in \mathcal{J}-\mathcal{J}^{E}$ and it is sufficient to prove that this $J^{\prime}$ is in the closure of $\mathcal{J}^{E}$. The proof will be by contradiction; namely the assumption that $J^{\prime}$ lies in the interior of $\mathcal{J}-\mathcal{J}^{E}$ will yield some nonsense. So, assume that $J^{\prime}$ is in the interior of this last set. Thus, each $J_{1}$ near to $J^{\prime}$ will admit an irreducible, finite energy pseudoholomorphic subvariety, $C_{1}$, which contains $A$. To arrive at the contradiction, associate to $J_{1}$ as described above the following:

$$
\mathcal{E}\left(J_{1}\right)=\inf \left\{\int_{*} \omega\right\}
$$


where the inf is taken over all irreducible, finite energy $J_{1}$-pseudoholomorphic subvarieties in $B_{4}$ which contain the region $A$. Note the $\mathcal{E}$ is necessarily defined and bounded by $E$ on some neighborhood of $J^{\prime}$. On the other hand, the assumption that $\mathcal{E}$ is defined on a neighborhood of $J^{\prime}$ will be shown to imply that $\mathcal{E}$ is unbounded on any neighborhood of $J^{\prime}$. This is the contradiction which proves Lemma 6.1 .

The conclusion that $\mathcal{E}$ is unbounded on any neighborhood of $J^{\prime}$ follows by iterating the following lemma:

LEMMA 6.2. There exists $e>0$ with the following significance: Let $J_{1}$ lie in the interior of $\mathcal{J}-\mathcal{J}^{E}$. Then, there exist elements in $\mathcal{J}$ which are as close as desired to $J_{1}$ in the $C^{\infty}$ topology, and which have $\mathcal{E} \geq \mathcal{E}\left(J_{1}\right)+e$.

Proof of Lemma 6.2. Observe first that if $J_{1} \in \mathcal{J}-\mathcal{J}^{E}$, then Proposition 3.8 in [T2] implies that there exists an irreducible, $J_{1}$-pseudoholomorphic subvariety $C_{1} \subset$ $B_{4}$ whose energy is equal to $\mathcal{E}\left(J_{1}\right)$ and which contains the region $A$. Consider now perturbing $J_{1}$ in a small ball which is centered on $C$, whose closure is contained in $B_{1}$, and whose intersection with $A$ is contained in a connected open set which intersects $A$. The assumption that $J_{1}$ is an interior point of $\mathcal{G}-\mathcal{G}_{E}$ implies that the perturbation, $J_{2}$, if sufficiently close to $J_{1}$ in the $C^{\infty}$ topology, comes with an irreducible, $J_{2}$ pseudoholomorphic subvariety of $B_{4}$ which contains $A$ and whose energy is no greater than $E$.

Take a sequence of such perturbed versions of $J_{1}$ which converges to $J_{1}$ in the $C^{\infty}$ topology. The corresponding sequence of irreducible, pseudoholomorphic subvarieties in $B_{4}$ will converge geometrically, and the limit will be a set of $J_{1}$-pseudoholomorphic subvarieties with positive multiplicities. (Again, this all follows from Proposition 3.8 in [T2].) Note that $C_{1}$ must appear in the set of such subvarieties as each of the elements of the sequence contains $A$ and $C_{1}$ contains $A$. If $C_{1}$ appears with multiplicity greater than 1 , or if a second subvariety appears in the limit set, then the energies of the elements in the sequence must be at least some fixed amount, $e$, greater than that of $C_{1}$. (The second subvariety intersects $B_{1}$ but must extend out to $B_{4}$ and so has a uniform lower bound to its energy.)

With this last point in hand, Lemma 6.2 can fail only if the following assertion is true:

Every sequence of subvarieties as just described converges geometrically, and the limit is $C_{1}$ with multiplicity one .

On the other hand, a sequence can be exhibited where the latter conclusion is impossible. The exhibition below of such a sequence completes the proof of Lemma 6.2. The exhibition of this promised sequence requires six steps.

Step 1: The perturbation of $J_{1}$ will occur in a ball $B^{\prime} \subset B_{1}$ of very small radius centered on $C$ and situated where $J_{1}=J$ and where $C_{1}$ coincides with $C$. Let $\delta>0$ denote the radius of $B^{\prime}$.

Let $D^{\prime}$ denote the intersection of $B^{\prime}$ with $C$. Then, $D^{\prime} \subset C_{1}$. Moreover, if the radius $\delta$ of $B^{\prime}$ is chosen small, then $D^{\prime}$ is an embedded disk. Furthermore there exists $c>0$ such that when $\delta<100 c$, there are complex coordinates $(\xi, \eta)$ on a neighborhood of $B^{\prime}$ in $\mathbb{R}^{4}$ with the following properties:

- $\left\{(\xi, \eta):|\xi|^{2}+|\eta|^{2} \leq \delta^{2} / 4 \subset B^{\prime} \subset\left\{(\xi, \eta):|\xi|^{2}+|\eta|^{2} \leq 4 \delta^{2}\right\}\right.$.

- The origin gives the center of $B^{\prime}$. 
- $\eta \equiv 0$ on $D^{\prime}$.

- When $|\kappa| \leq C$, then the disks $\Sigma_{\kappa} \equiv\{\xi=\kappa,|\eta|<c\}$ are $J$-pseudoholomorphic.

- The complex valued 1 -form $d \xi$ and $d \eta$ have norm $\sqrt{ } 2+\mathcal{O}(|\eta|+|\xi|)$ and their projections into the $T^{0,1}$ summand of $T B^{\prime}$ has norm $\mathcal{O}(|\eta|)$.

(See, e.g. Lemma 5.4 in [T5].)

Step 2: To define the perturbations of $J_{1}$, first observe that the tangent space

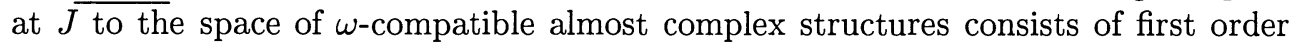
deformations of $J$ of the form [J, S], where $S^{T}=S$. That is, $S$ is a symmetric, $4 \times 4$ matrix. In this case, take $S=s \sigma I$, where $s$ is a real number between 0 and 1 , where $\sigma$ is a smooth function on $B^{\prime}$ with compact support, and where $I$ is a $4 \times 4$ symmetric matrix which has only its $\mathrm{t}-\mathrm{x}$ and $\mathrm{r}$-t entries non-zero. In particular, require $I \cdot \partial_{t}=\partial_{x}$ and $I \cdot \partial_{x}=\partial_{t}$.

To be precise about $\sigma$, choose a fixed function $\chi:[0, \infty) \rightarrow[0,1]$ which equals one near 0 and zero near one. Then, set $\sigma(\chi, \eta) \equiv \chi\left(10\left(|\xi|^{2}+|\eta|^{2}\right) / \delta^{2}\right)$.

Varying $s$ towards 0 gives a sequence of first order deformations of $J_{1}$ which converges to $J_{1}$ in the $C^{\infty}$ topology. This first order sequence should then be exponentiated to obtain the desired sequence of almost complex structures which converges to $J_{1}$. This sequence will be denoted by $\left\{J_{1 s}\right\}$, where $J_{1 s}=J_{1}+s \sigma\left[J_{1}, I\right]+\mathcal{O}\left(s^{2} \sigma^{2}\right)$.

Step 3: Perturb $J_{1}$ to $J_{1 s}$. If the assertion in (6.2) is true, then there must exist, for fixed $\delta$ and then for each sufficiently small and positive $s, a J_{1 s}$-pseudoholomorphic subvariety $D_{s}^{\prime} \subset B^{\prime}$ which agrees with $D^{\prime}$ on the compliment of the support of the function $\sigma$. Moreover, as $s \rightarrow 0$, the sequence $\left\{D_{s}^{\prime}\right\}$ must converge to $D^{\prime}$. These last conditions imply that each $D_{s}^{\prime}$ is an embedded disk which can be written as a graph over $D^{\prime}$. This is to say that each $D_{s}^{\prime}$ (for small and positive $s$ ) is parameterized by $\kappa \in D^{\prime}$ via $\left(\zeta=\kappa, \eta=f_{s}(\kappa)\right)$. Here, $f_{s}$ is a smooth function. Also, be aware that $\sup _{D^{\prime}}\left|f_{s}\right| \rightarrow 0$ as $s \rightarrow 0$.

The argument for the assertion that $D_{s}^{\prime}$ is a graph over $D^{\prime}$ proceeds as follows: If $\kappa \in D^{\prime}$, then the submanifold $\Sigma_{\kappa}$ intersects $D^{\prime}$ exactly once. Meanwhile, for small $s$, each such $\Sigma_{\kappa}$ can be perturbed to give a family of $J_{1 s}$-pseudoholomorphic submanifolds of $B^{\prime}$ parameterized by the points in $D^{\prime}$. Moreover, the perturbed version of $\Sigma_{\kappa}$ can be assumed to pass through the point $\kappa$ in $D^{\prime}$ and to agree with the original version when $\kappa$ is near the edge of $D^{\prime}$. Indeed, a slight modification of the arguments for Lemma 5.5 of [T5] will produce this family of perturbations.

Now, for $\kappa$ near the edge of $D^{\prime}$, the corresponding perturbation of $\Sigma_{\kappa}$ has intersection number 1 with $D_{s}^{\prime}$ as the latter agrees there with $D^{\prime}$. Thus, if $D_{s}^{\prime}$ is everywhere close to $D^{\prime}$, then each perturbed version of $\Sigma_{\kappa}$ will have intersection number 1 with $D_{s}^{\prime}$. As both of these varieties are $J_{1 s}$-pseudoholomorphic, it follows that they have geometric intersection number 1 as well. Thus, $D_{s}^{\prime}$ is realized as a graph over $D^{\prime}$.

Step 4: Given that $D_{s}^{\prime}$ is a graph over $D^{\prime}$, the assertion that $D_{s}^{\prime}$ is $J_{1 s}$-pseudoholomorphic translates into a certain non-linear differential equation for the function $f_{s}$. In particular, this function obeys an equation having the schematic form

$$
\bar{\partial} f_{s}+v f_{s}+\mu \bar{f}_{s}=s \underline{\sigma}+\mathcal{R} .
$$

Here, $v$ and $\mu$ are determined by the 1-jet of $J$ along $C$ and thus bounded in norm by a constant which is independent of the radius, $\delta$ of $B^{\prime}$ and of the parameter $s$. Meanwhile, $\underline{\sigma}$ is, up to a factor of 2 and a sign, just the restriction of $\sigma$ to $D^{\prime}$. Finally, 
for small $\delta$ and sufficiently small $s$, the terms which comprise $\mathcal{R}$ in (6.3) obey the bound

$$
|\mathcal{R}| \leq \zeta\left(s \delta+s^{2}+\left|f_{s}\right|^{2}+\left|f_{s}\right|\left|\nabla f_{s}\right|\right)
$$

Here, $\zeta$ is a constant which is independent of $\delta$ and $s$. in $D^{\prime}$.

Note that of interest here are those solutions to (6.4) which have compact support

Step 5: Square both sides of (6.4) and integrate over $D^{\prime}$. After an integration by parts to equate $\int\left|\bar{\partial} f_{s}\right|^{2}$ with $4^{-1} \int\left|d f_{s}\right|^{2}$, some standard inequalities for the Dirichlet integral can be invoked. (Since $f_{s}$ has compact support in $D^{\prime}$, there is nothing to hinder either the integration by parts or the inequalities that will be subsequently invoked.) In particular, given that $f_{s}$ has compact support in $D^{\prime}$ and has small norm when $s$ is small, and given (6.5), one can deduce that

$$
\int_{D^{\prime}}\left(\delta^{-2}\left|f_{s}\right|^{2}+\left|d f_{s}\right|^{2}\right) \leq \zeta \delta^{2} s^{2}
$$

where $\zeta$ is, once again, independent of $\delta$ and $s$.

Step 6: To see that the assertion in (6.2) cannot hold for the sequence $\left\{J_{1 s}\right\}$, it is sufficient to prove the following: When $\delta$ is small though positive, and when $s$ is positive, but very small, no compactly supported function $f_{s}$ satisfies both (6.4) and (6.6). Indeed, if $f_{s}$ satisfies (6.4), (6.6) and has compact support, then an integration of both sides of (6.4) yields the equality

$$
\int_{D^{\prime}}\left(v f_{s}+\mu \bar{f}_{s}\right)=s \int_{D^{\prime}} \underline{\sigma}+\mathcal{O}\left(\delta^{3} s+\delta^{2} s^{2}\right)
$$

(Here, (6.5) has been used to obtain the error term on the right hand side.) In particular, note that for small $\delta$, and then for very small $s$, the integral term on the right hand side of (6.7) has absolute value which is greater than a certain $\delta$ and $s$ independent multiple of $s \delta^{2}$. Thus, $s \delta^{2}$ gives the size (in absolute value) of the right hand side of (6.7) when $\delta$ is small and then $s$ is very small.) Meanwhile, according to (6.6), when $\delta$ is small and then $s$ is very small, the left hand side of (6.7) is no greater than a uniform multiple of $\delta^{3} s$. Thus, (6.7) cannot hold for small $\delta$ and then very small $s$.

b) Proposition 2.12. Once the map, $\psi$, from a ball into $\mathcal{G}$ is fixed, one can use a continuous family of isotopies of $X$ (parameterized by the ball) to obtain a new map, $\psi^{\prime}$, which parameterizes closed 2-forms which all have the identical vanishing set $Z$. Then, the metrics and forms which are parameterized by $\psi^{\prime}$ will all agree on some tubular neighborhood of $Z$. In this regard, be aware that $\mathcal{G}$ decomposes into components which are classified, at least in part, by isotopy classes of the circles which comprise the set $Z$.

Note that this family of isotopies which turns $\psi$ into $\psi^{\prime}$, though parameterized by the given ball, need not yield the trivial isotopy when the parameterizing point lies on the boundary of the ball. In any event, once the map $\psi^{\prime}$ is perturbed to a map into $\mathcal{G}^{\prime}$, the isotopies can be run backward on this perturbation to obtain a perturbation of the given map $\psi$ which agrees with $\psi$ on the boundary of the parameterizing ball, yet lands fully in $\mathcal{G}^{\prime}$. 
With the preceding understood, one can henceforth assume with out losing any generality that all the pairs $(g, \omega)$ which are parameterized by the map $\psi$ have the same $Z$ and are identified in some neighborhood of this $Z$. Then, the preceding proof of Proposition 2.11 also proves Proposition 2.12 in that the argument for the former concerned a perturbation which was localized to any sufficiently small radius ball near $Z$ with center on the $t-z$ plane.

\section{REFERENCES}

[Ad] R. AdAms, Sobolev Spaces, Academic Press, 1975.

[Gr] M. Gromov, Pseudoholomorphic curves in symplectic manifolds, Invent. Math., 82 (1985), pp. 307-347.

[H] H. Hofer, In the Proceedings of the International Congress of Mathematician, Berlin, 1998, Vol I, in Documenta Mathematica Extra Volume ICM, 1998.

[HWZ] H. HoFER, K. WYSOCKI, AND E. ZEHNDER, Properties of pseudoholomorphic curves in symplectisations I: Asymptotics, Analyse Nonlinear, 13 (1996), pp. 337-379.

[Ho] K. HoNDA, Harmonic forms for generic metrics, preprint 1997.

[HL1] M. Hutchings AND Y. J. LeE, Circle-valued Morse theory, R-torsion and Seiberg-Witten invariants of 3-manifolds, Topology, to appear.

[HL2] M. Hutchings AND Y. J. LeE, Circle-valued Morse theory and Rademeister torsion, preprint 1997.

[Lu] C. LUTTINGER, private communication.

[McD] D. MCDuFf, Singularities and positivity of intersections of J-holomorphic curves, with Appendix by Gang Liu, in the Proceedings of CIMPA Summer School of Symplectic Topology, Nice, 1992, Birkhauser, 1994.

[MS] D. MCDUfF AND D. SALAMON, J-holomorphic Curves and Quantum Cohomology, AMS University Lecture Series, 6, American Math Society, 1996.

[Pan] P. PANsu, Pseudoholomorphic curves in symplectic manifolds, in Holomorphic Curves in Symplectic Geometry, M. Audin and F. Lafontaine, ed., Progress in Math, 117, Birkhasuer, 1994.

[Mo] C. B. Morrey, Multiple Integrals in the Calculus of Variations, Springer-Verlag, 1966.

[PW] T. PARker and J. Wolfson, Pseudoholomorphic maps and bubble trees, J. Goem. Anal., 3 (1993), pp. 63-98.

[T1] C. H. TAUBES, Seiberg-Witten invariants and pseudoholomorphic subvarieties for self-dual, harmonic 2-forms, preprint 1998.

[T2] C. H. TAUBES, The structure of pseudoholomorphic subvarieties for a degenerate almost complex structure on $S^{1} \times B^{3}$, Geometry and Topology, 2 (1998) 221-332.

[T3] C. H. TAubes, The geometry of the Seiberg-Witten invariants, in Surveys in Differential Geometry, S.T. Yau, ed., International Press, 1996.

[T4] C. H. TAUBES, The geometry of the Seiberg-Witten invariants, in the Proceedings of the International Congress of Mathematician, Berlin 1998 Vol II, in Documenta Mathematica Extra Volume ICM, 1998, pp. 493-506.

[T5] C. H. TAubes, $S W \Rightarrow G r:$ From the Seiberg-Witten equations to pseudoholomorphic curves, J. Amer. Math. Soc., 9 (1996), pp. 845-918. (See also the revised version in The Proceeding of the First IP Lecture Series Vol II, R. Wentworth, ed., International Press, to appear.)

[T6] C. H. TAUBES, GR $\Rightarrow S W$ : From pseudoholomorphic curves to Seiberg-Witten solutions, preprint 1997.

[Tu] V. TURAEV, A combinatorial formula for the Seiberg-Witten invariants of 3-manifolds, preprint 1998.

[Ye] R. YE, Gromov's compactness theorem for pseudoholomorphic curves, Trans. Amer. Math. Soc., 342 (1994), pp. 671-694. 